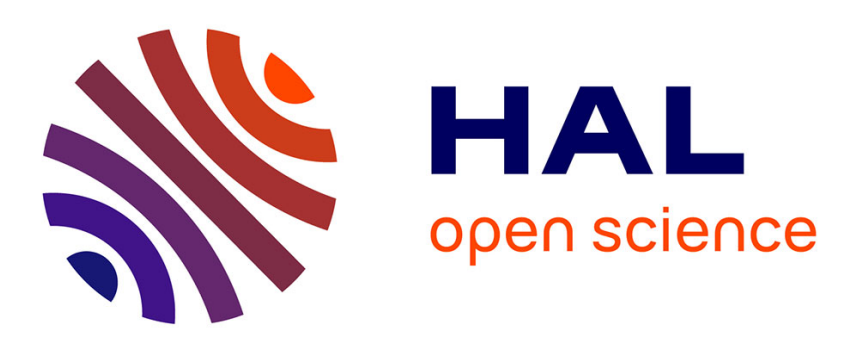

\title{
Solving 2D linear isotropic elastodynamics by means of scalar potentials: a new challenge for finite elements
} Jorge Albella Martínez, Sébastien Imperiale, Patrick Joly, Jerónimo Rodríguez

\section{To cite this version:}

Jorge Albella Martínez, Sébastien Imperiale, Patrick Joly, Jerónimo Rodríguez. Solving 2D linear isotropic elastodynamics by means of scalar potentials: a new challenge for finite elements. Journal of Scientific Computing, 2018, 10.1007/s10915-018-0768-9 . hal-01803536

\section{HAL Id: hal-01803536 \\ https://hal.inria.fr/hal-01803536}

Submitted on 30 May 2018

HAL is a multi-disciplinary open access archive for the deposit and dissemination of scientific research documents, whether they are published or not. The documents may come from teaching and research institutions in France or abroad, or from public or private research centers.
L'archive ouverte pluridisciplinaire $\mathbf{H A L}$, est destinée au dépôt et à la diffusion de documents scientifiques de niveau recherche, publiés ou non, émanant des établissements d'enseignement et de recherche français ou étrangers, des laboratoires publics ou privés. 


\title{
Solving 2D linear isotropic elastodynamics by means of scalar potentials: a new challenge for finite elements
}

\author{
Jorge Albella Martínez ${ }^{1}$, Sébastien Imperiale ${ }^{2,3}$, Patrick Joly ${ }^{2,4}$, \\ and Jerónimo Rodríguez ${ }^{1}$ \\ ${ }^{1}$ Departamento de Matemática Aplicada, Universidade de Santiago \\ de Compostela, 15706 Santiago de Compostela, Spain \\ ${ }^{2}$ Inria, Université Paris-Saclay, France \\ ${ }^{3}$ LMS, Ecole Polytechnique, CNRS, Université Paris-Saclay, France \\ ${ }^{4}$ UMA, Ensta, CNRS, Université Paris-Saclay, France \\ ${ }^{5}$ IMAT, Universidade de Santiago de Compostela, 15706 Santiago \\ de Compostela, Spain \\ ${ }^{6}$ ITMATI, Campus Sur, 15706 Santiago de Compostela, Spain
}

May 7, 2018

\begin{abstract}
In this work we present a method for the computation of numerical solutions of $2 \mathrm{D}$ homogeneous isotropic elastodynamics equations by solving scalar wave equations. These equations act on the potentials of a Helmholtz decomposition of the displacement field and are decoupled inside the propagation domain. We detail how these equations are coupled at the boundary depending on the nature of the boundary condition satisfied by the displacement field. After presenting the case of rigid boundary conditions, that presents no specific difficulty, we tackle the challenging case of free surface boundary conditions that presents severe stability issues if a straightforward approach is used. We introduce an adequate functional framework as well as a time domain mixed formulation to circumvent these issues. Numerical results confirm the stability of the proposed approach.
\end{abstract}

\section{Introduction}

In this paper our goal is to revisit a very classical question, namely, the numerical solution of elastodynamics equations in isotropic media, which govern the 
propagation of elastic waves in solids, in the time domain. As a matter of fact there exist already many numerical methods for solving these equations. To begin with, for instance, in the framework of finite elements in space and finite differences in time, standard conforming finite elements (possibly high order) methods for the pure displacement formulation of the elastodynamics system (a second order hyperbolic system) as well as mixed finite element methods for the equivalent velocity-stress formulation of the same system (first order differential system). This space discretization is then coupled to explicit finite difference time stepping that is subject to a CFL stability condition.

On the other hand, in many classical physics text books authors used the well-known Helmholtz decomposition of vector fields (write a vector field as the sum of a gradient and a curl) to compute analytical solutions in homogeneous isotropic media. Such a decomposition relates elastodynamic equations to two wave equations and enlightens the decomposition of the wave field as the sum of pressure waves (P-waves, that are gradients of a pressure potential $\left.\varphi_{P}\right)$ and shear waves (S-waves, that are curls of a shear potential $\varphi_{S}$ ) that propagate independently with different velocities, the velocity $V_{P}$ of the $\mathrm{P}$-waves being larger than $V_{S}$ the velocity of the $\mathrm{S}$-waves. In the $2 \mathrm{D}$ case, to which we will restrict ourselves for simplicity, the simplification is that both pressure and shear potentials are scalar. However the extension to 3D does not pose a priori any additional conceptual difficulty and will be the object of further developments.

In a piecewise homogeneous media, such a decomposition is valid locally and the different types of waves recouple at boundaries and interfaces. This is the main source of complexity of the propagation process. Looking at the literature, it seems that very few works have been devoted on the exploitation of this idea for finite element computations (however, one can find a few references concerning finite differences computations, see 1 in which a finite difference scheme is constructed with approximation properties independent of the ratio $\left.V_{P} / V_{S}\right)$ although it has been used in other domains of physics, in particular in fluid mechanics (current-vorticity formulations [2], chapter 2, [3] and [4]). The first motivation of the present work is an intellectual curiosity: could we use potentials to solve isotropic elastodynamics with finite elements? There is also a more relevant motivation concerning applications. This would concern the propagation of elastic waves in nearly incompressible media, such as soft tissues, in which $\mathrm{P}$-waves propagate much faster than S-waves. In such a case, it is well known that displacement-based methods, which do not distinguish both waves along the calculation process, are greatly penalized by large values of the ratio $V_{P} / V_{S}$ due to the CFL condition (assuming that explicit time integrators are used). Let us explain this by a simple computation. Let us consider a ddimensional isotropic homogeneous medium of characteristic length $L$ on each direction, subject to a source term involving a minimal time scale $T_{\star}$. This source generates two different minimal wavelenghts, the $S$-wavelength $\lambda_{S}=$ $V_{S \star}$, which is much smaller than the $P$-wavelength $\lambda_{P}=V_{P \star}$ if $V_{P} / V_{S}$ is large. Assuming that we consider $\mathbb{P}_{1}$ or $\mathbb{Q}_{1}$ finite elements on a quasi-regular mesh of step size $h$, for accuracy reasons, $h$ should be chosen proportional to $\lambda_{S}$, that is, $h \propto \lambda_{S}$. On the other hand, considering a leap-frog time discretization for 
instance, the time step is constrained by the stability condition that involves the fastest velocity $V_{P}$, that is, $\Delta t \propto h / V_{P}$. Considering a time interval integration $[0, T]$, the number of time steps is $T / \Delta t$ and since, one uses an explicit scheme, the cost of each iteration is proportional to number of degrees of freedom, namely $L^{d} / h^{d}$. As a consequence, we can roughly estimate the overall computational cost as

$$
\text { Cost } \propto \frac{L^{d} T}{h^{d} T_{\star}} \propto \frac{L^{d}}{\lambda_{S}^{d}} \frac{T}{T_{\star}} \frac{V_{P}}{V_{S}},
$$

where $L^{d} / \lambda_{S}^{d}$ represents the size of the problem in space and $T / T_{\star}$ the size of the simulation in time. Clearly the penalizing factor is $V_{P} / V_{S}$ which would not appear when solving a standard scalar wave equation.

Potential formulations will a priori authorize the use of different meshes for both the pressure and shear potentials in view of adapting the mesh size to each wave length which is smaller for $S$ than for $P$ waves which would result in an important saving of the computation cost for large ratio $V_{P} / V_{S}$. This will be explained with more details in Section 2.3. A by product of this approach is that one could benefit of well-known techniques for the numerical treatment of the standard scalar wave equation such as for example the use of perfectly match layers (PMLs) for the treatment of unbounded domains. Indeed long time stable implementations of PMLs for isotropic elastodynamic equations raise some difficulties especially if the ratio $V_{P} / V_{S}$ is large (even though the so-called C-PML solve the long time stability issue as shown in [5]).

As the reader can expect, the main source of difficulties is the treatment of boundaries and interfaces because, contrary to the interior equations, boundary and transmission conditions are not easily expressed in terms of these potentials. The main goal and the main challenge we wish to address in this paper is the treatment of the couplings induced by these various surface conditions, that we need to handle in a guaranteed stable way and possibly with hopefully no influence on the CFL condition after time discretization. In [6, 7] we first addressed the case of a homogeneous medium with a clamped boundary, that is to say the Dirichlet boundary condition, for which we succeeded in achieving this goal. The approach and results of this paper will be recalled in Section 2. This second paper follows the philosophy of the previous work and aims at treating the free surface boundary condition, or Neumann boundary condition, that appears as much more challenging. This work is also preparatory to the treatment of interface conditions: there are two of them, one is of Dirichlet type, the second one of Neumann type and we anticipate that their treatment would rely on both treatments of Dirichlet and Neumann boundary conditions.

The outline of the rest of the article is as follows. In Section 2, we first shall recap how to reduce the solution of $2 \mathrm{D}$ isotropic elastodynamics equations to two scalar wave equations and more importantly, explain how to treat the Dirichlet boundary condition as it has been done in [6]. The main section of this paper is Section 3 where we treat the free surface boundary condition. In Sect. 3.2 we show that the most naive approach directly inspired from the treatment of the Dirichlet condition gives rise to serious numerical stability problems af- 
ter discretization. This is linked to the proposed variational formulation of the continuous model, the apparently natural functional space being too large and authorizing the development of unstable surface modes after space discretization. More precisely it appears that the new mass bilinear form which contains an additional boundary term (this is the main difference between Dirichlet and Neumann problems) fails to be positive contrary to the stiffness matrix (which remains the same as for the Dirichlet condition). So the key idea for the circumventing the problem is to find a smaller (but still sufficiently large) variational space in which we recover the positivity of the mass bilinear form. This is precisely the object of Section 3.3 . For the construction of this space, we are guided by the comparison of the energy naturally associated to the new potentials formulation with the classical elastic energy associated to the displacement formulation. The definition of this new space is quite implicit and involves the solution of some elastostatic problem. Fortunately, such a space can be characterized as the orthogonal (with respect to the new mass bilinear form) of another subspace which is itself isomorphic to a space of scalar functions defined on the boundary. We can exploit this characterization by proposing a mixed variational formulation in which the above-mentioned orthogonality relation is treated as a constraint leading to the introduction of Lagrange multipliers as functions defined along the boundary. The resulting formulation is proven to be stable: this is the major achievement of this paper. Finally we show some numerical experiments that confirm the theoretical results previously obtained.

\section{Decomposition into potentials: the case of a Dirichlet boundary condition}

This section has been added for pedagogical purpose, for making the paper self contained and for preparing Section 3. Section 2.1 recaps very standard material while Sections 2.2 and 2.3 are a summary to what has been done in $[6]$.

\subsection{Decomposition into potentials in homogeneous media}

Preliminary notation. Throughout the paper we will work in $2 \mathrm{D}$ and $\boldsymbol{x}=$ $\left(x_{1}, x_{2}\right)$ will denote the space variable. We shall use bold letters for representing vector fields such as $\boldsymbol{u}=\left(u_{1}, u_{2}\right)$ for the displacement field in a elastic body or $\boldsymbol{v}=\left(v_{1}, v_{2}\right)$ for the velocity field $\left(\boldsymbol{v}=\partial_{t} \boldsymbol{u}\right)$. Ordinary letters will be used for scalar fields such as the components of the vector fields or the forthcoming potentials to be introduced. Finally, underlined bold letters will be used for $2 \times 2$ tensor fields such as the deformation or strain tensor $\underline{\varepsilon}(\boldsymbol{u})=\left(\left(\varepsilon_{i j}(\boldsymbol{u})\right)\right)$

where $1 \leq i, j \leq 2$ or the stress tensor $\underline{\boldsymbol{\sigma}}=\left(\left(\sigma_{i j}\right)\right)$ that represents the internal efforts inside the body. 
Mathematical model. Let us briefly recap the 2D elastodynamics equations. First, the time variation of the displacement field $\boldsymbol{u}$ is governed by the fundamental law in mechanics

$$
\rho \partial_{t}^{2} \boldsymbol{u}-\operatorname{div} \underline{\boldsymbol{\sigma}}=\boldsymbol{f},
$$

where $\operatorname{div} \underline{\boldsymbol{\sigma}}$ is the vector field defined by

$$
(\boldsymbol{d i v} \underline{\boldsymbol{\sigma}})_{i}=\partial_{j} \sigma_{i j}(\boldsymbol{u}),
$$

(with Einstein's convention for summation over the repeated indices) $\rho=\rho(\boldsymbol{x}) \geq$ $\rho_{0}>0$ is the density of the body that might depend on the $\boldsymbol{x}$ variable for heterogeneous media and the source term $\boldsymbol{f} \in L_{\mathrm{loc}}^{1}\left(\mathbb{R}^{+},\left(L^{2}(\Omega)\right)^{2}\right)$. Equation (2) must be completed by constitutive laws that relates the displacement field to the stress tensor. In an isotropic medium this is given by Hooke's law which involves the (non negative) Lamé parameters $\lambda(\boldsymbol{x})$ and $\mu(\boldsymbol{x})$

$$
\underline{\boldsymbol{\sigma}}=\underline{\boldsymbol{\sigma}}(\boldsymbol{u}):=\lambda \operatorname{div} \boldsymbol{u} \underline{\boldsymbol{I}}+2 \mu \underline{\boldsymbol{\varepsilon}}(\boldsymbol{u}),
$$

where $\underline{\boldsymbol{I}}$ is the $2 \times 2$ identity matrix and (we use again Einstein's convention)

$$
\operatorname{div} \boldsymbol{u}=\partial_{j} u_{j}, \quad \varepsilon_{i j}(\boldsymbol{u})=\frac{1}{2}\left(\partial_{i} u_{j}+\partial_{j} u_{i}\right), 1 \leq i, j \leq 2 .
$$

One can eliminate the unknown $\underline{\sigma}(\boldsymbol{u})$ by substituting (3) in (2) and obtain a second order system in $\boldsymbol{u}$. In the homogeneous case, i.e. when $\lambda, \mu$ and $\rho$ are constant, we easily compute that

$$
\operatorname{div}(\underline{\boldsymbol{\sigma}}(\boldsymbol{u}))=(\lambda+2 \mu) \nabla \operatorname{div} \boldsymbol{u}-\mu \operatorname{curl}(\operatorname{curl} \boldsymbol{u}),
$$

so that the equations can be written as follows (see [8, 9, for instance)

$$
\rho \partial_{t}^{2} \boldsymbol{u}-(\lambda+2 \mu) \nabla \operatorname{div} \boldsymbol{u}+\mu \operatorname{curl}(\operatorname{curl} \boldsymbol{u})=\boldsymbol{f},
$$

where we have introduced the two curl operators in $2 \mathrm{D}$ defined by

$$
\begin{array}{ll}
\operatorname{curl} \boldsymbol{u}:=\partial_{1} u_{2}-\partial_{2} u_{1}, & \text { for the scalar curl of a vector field } \boldsymbol{u}, \\
\operatorname{curl} \varphi:=\left(\partial_{2} \varphi,-\partial_{1} \varphi\right), & \text { for the vector curl of a scalar field } \varphi .
\end{array}
$$

Equation (5) is completed, in the presence of boundaries, with boundary conditions (see later) and, for the sake of simplicity, vanishing initial conditions

$$
\boldsymbol{u}(t=0)=\mathbf{0}, \quad \partial_{t} \boldsymbol{u}(t=0)=\mathbf{0} .
$$

Decomposition into potentials. We are now going to introduce two scalar potentials $\varphi_{P}$ and $\varphi_{S}$ that realize a Helmholtz decomposition, when there is no source term, of the velocity field $\boldsymbol{v}=\partial_{t} \boldsymbol{u}$,

$$
\rho \partial_{t} \varphi_{P}=(\lambda+2 \mu) \operatorname{div} \boldsymbol{u}, \quad \rho \partial_{t} \varphi_{S}=-\mu \operatorname{curl} \boldsymbol{u} .
$$


Substituting (8) into (5) leads, to $\partial_{t}\left(\boldsymbol{v}-\nabla \varphi_{P}-\operatorname{curl} \varphi_{S}-\boldsymbol{g}\right)=0$ where

$$
\boldsymbol{g}(t)=\frac{1}{\rho} \int_{0}^{t} \boldsymbol{f}(s) d s
$$

Imposing vanishing initial conditions for the potentials

$$
\varphi_{P}(t=0)=0, \quad \varphi_{S}(t=0)=0,
$$

we get (since $\boldsymbol{v}(t=0)=\mathbf{0})$

$$
\boldsymbol{v}=\nabla \varphi_{P}+\operatorname{curl} \varphi_{S}+\boldsymbol{g}
$$

which provides a Helmholtz decomposition 10 of the vector field $\boldsymbol{v}$ when $\boldsymbol{g}$ vanishes. To obtain the equations satisfied by the potentials we simply substitute (11) into the two equations in (8) differentiated in time to get two scalar wave equations for $\varphi_{P}$ and $\varphi_{S}$

$$
\frac{1}{V_{P}^{2}} \partial_{t}^{2} \varphi_{P}-\Delta \varphi_{P}=\operatorname{div} \boldsymbol{g}, \quad \frac{1}{V_{S}^{2}} \partial_{t}^{2} \varphi_{S}-\Delta \varphi_{S}=-\operatorname{curl} \boldsymbol{g},
$$

with $V_{P}\left(\right.$ resp. $\left.V_{S}\right)$ the velocity of the P-waves (resp. $S$-waves) defined by

$$
V_{P}=\sqrt{\frac{\lambda+2 \mu}{\rho}}, \quad \mathrm{P} \text { waves velocity, } \quad V_{S}=\sqrt{\frac{\mu}{\rho}}, \quad \mathrm{S} \text { waves velocity. }
$$

From (8) we obtain the initial conditions for the time derivative of the potentials

$$
\partial_{t} \varphi_{P}(t=0)=0, \quad \partial_{t} \varphi_{S}(t=0)=0 .
$$

Note that in the free space (in absence of any boundary), the two wave equations in (12) are fully decoupled.

\subsection{Decomposition into potentials for a clamped domain}

We now consider a $2 \mathrm{D}$ homogeneous isotropic propagation domain $\Omega \varsubsetneqq \mathbb{R}^{2}$, for instance, $\Omega$ bounded, with boundary $\Gamma=\partial \Omega$ that we assume to be clamped which means that equations (5) are completed with the boundary condition

$$
\boldsymbol{v}=\mathbf{0}, \quad \text { in } \Gamma .
$$

Proceeding as in the previous section, we introduce $\varphi_{P}$ and $\varphi_{S}$ via equations (8) so that, inside $\Omega$, the Helmholtz decomposition (11) holds and the potentials satisfy the scalar wave equations in $(12)$. These equations must be completed by boundary conditions traducing 15 . We assume in the following that $\Gamma$ is a finite union of piecewise $C^{1}$ closed curves and thus admits almost everywhere a unit normal outward vector $\boldsymbol{n}$ and unit tangent vector $\boldsymbol{\tau}$ in such a way that the frame $(\boldsymbol{\tau}, \boldsymbol{n})$ is a direct frame so that, if $\boldsymbol{n}=\left(n_{1}, n_{2}\right)$, then $\boldsymbol{\tau}=\left(n_{2},-n_{1}\right)$. For 
any sufficiently smooth scalar field $\varphi$ we have the following identities for traces in $\Gamma$

$$
\operatorname{curl} \varphi \cdot \boldsymbol{n}=-\partial_{\boldsymbol{\tau}} \varphi, \quad \operatorname{curl} \varphi \cdot \boldsymbol{\tau}=\partial_{\boldsymbol{n}} \varphi
$$

where as usual $\partial_{\boldsymbol{n}} \varphi=\nabla \varphi \cdot \boldsymbol{n}$ and $\partial_{\boldsymbol{\tau}} \varphi=\nabla \varphi \cdot \boldsymbol{\tau}$. Thus, writing that $\boldsymbol{v}=\mathbf{0}$ is equivalent to writing $\boldsymbol{v} \cdot \boldsymbol{n}=0$ and $\boldsymbol{v} \cdot \boldsymbol{\tau}=0$, which leads, according to (11), to the following boundary conditions for $\varphi_{P}$ and $\varphi_{S}$

$$
\partial_{\boldsymbol{n}} \varphi_{P}=\partial_{\boldsymbol{\tau}} \varphi_{S}-\boldsymbol{g} \cdot \boldsymbol{n}, \quad \partial_{\boldsymbol{n}} \varphi_{S}=-\partial_{\boldsymbol{\tau}} \varphi_{P}-\boldsymbol{g} \cdot \boldsymbol{\tau}
$$

Note that the two essential conditions (15) for the displacement formulation become two natural conditions that couple the two potentials $\varphi_{P}$ and $\varphi_{S}$.

\subsection{A numerical approach for the Dirichlet problem: A recap}

Variational formulation. We first recall how to establish a weak formulation for the boundary value problem (12, 17). Assuming that the solution is sufficiently smooth, we can multiply the equations 12 by test functions $\psi_{P}$ and $\psi_{S}$ in $H^{1}(\Omega)$, integrate by parts and use $(17)$ to replace the normal derivatives of the potentials by tangential derivatives. After summation of the two resulting equations we can propose a first abstract variational formulation of the problem. To do so we introduce $\boldsymbol{\varphi}=\left(\varphi_{P}, \varphi_{S}\right)$ and $\boldsymbol{\psi}=\left(\psi_{P}, \psi_{S}\right)$ to get

$$
\left\{\begin{array}{l}
\text { Find } \boldsymbol{\varphi}(t): \mathbb{R}^{+} \longrightarrow H^{1}(\Omega)^{2} \text { such that }\left(\boldsymbol{\varphi}, \partial_{t} \boldsymbol{\varphi}\right)(t=0)=(\mathbf{0}, \mathbf{0}) \text { and } \\
\frac{\mathrm{d}^{2}}{\mathrm{~d} t^{2}} m_{\Omega}(\boldsymbol{\varphi}(t), \boldsymbol{\psi})+a(\boldsymbol{\varphi}(t), \boldsymbol{\psi})=l(t, \boldsymbol{\psi}), \quad \forall \boldsymbol{\psi} \in H^{1}(\Omega)^{2},
\end{array}\right.
$$

where the linear form $l(t, \cdot)$ is given by

$$
l(t, \boldsymbol{\psi})=-\int_{\Omega} \boldsymbol{g} \cdot\left(\nabla \psi_{P}+\operatorname{curl} \psi_{S}\right) \mathrm{d} \boldsymbol{x},
$$

and the mass bilinear form $m_{\Omega}(\cdot, \cdot)$ decouples $\varphi_{P}$ and $\varphi_{S}$

$$
\left\{\begin{array}{l}
m_{\Omega}(\boldsymbol{\varphi}, \boldsymbol{\psi})=m_{P}\left(\varphi_{P}, \psi_{P}\right)+m_{S}\left(\varphi_{S}, \psi_{S}\right) \\
m_{Q}\left(\varphi_{Q}, \psi_{Q}\right)=\frac{1}{V_{Q}^{2}} \int_{\Omega} \varphi_{Q} \psi_{Q} \mathrm{~d} \boldsymbol{x}, \quad Q \in\{P, S\}
\end{array}\right.
$$

The stiffness bilinear form $a(\cdot, \cdot)$ is given by

$$
a(\boldsymbol{\varphi}, \boldsymbol{\psi})=a_{\Omega}(\boldsymbol{\varphi}, \boldsymbol{\psi})+a_{\Gamma}(\boldsymbol{\varphi}, \boldsymbol{\psi})
$$

where the volumic bilinear form $a_{\Omega}(\cdot, \cdot)$ decouples in the same way as $m_{\Omega}(\cdot, \cdot)$

$$
\left\{\begin{array}{l}
a_{\Omega}(\boldsymbol{\varphi}, \boldsymbol{\psi})=a_{P}\left(\varphi_{P}, \psi_{P}\right)+a_{S}\left(\varphi_{S}, \psi_{S}\right) \\
a_{Q}\left(\varphi_{Q}, \psi_{Q}\right)=\int_{\Omega} \nabla \varphi_{Q} \cdot \nabla \psi_{Q} \mathrm{~d} \boldsymbol{x}, \quad Q \in\{P, S\}
\end{array}\right.
$$


and the coupling surface bilinear form $a_{\Gamma}(\cdot, \cdot)$ is defined by

$$
a_{\Gamma}(\boldsymbol{\varphi}, \boldsymbol{\psi})=\int_{\Gamma}\left(\partial_{\boldsymbol{\tau}} \varphi_{P} \psi_{S}-\partial_{\boldsymbol{\tau}} \varphi_{S} \psi_{P}\right) \mathrm{d} \boldsymbol{\gamma}
$$

where the integrals in the boundary should be interpreted as duality products between elements in $H^{\frac{1}{2}}(\Gamma)$ and its dual $H^{-\frac{1}{2}}(\Gamma)$. All the above bilinear forms are symmetric (for $a_{\Gamma}(\cdot, \cdot)$ use integration by parts along the boundary), however in order that (18) fits the classical theory of second order partial differential equations [11, some adequate positivity / coercivity for the forms $m_{\Omega}(\cdot, \cdot)$ and $a(\cdot, \cdot)$ need to be checked. The positivity of $m_{\Omega}(\cdot, \cdot)$ is clear but the positivity of the $a(\cdot, \cdot)$ is not obvious from (21) but relies on the following lemma

Lemma 2.1. One has the identity: for any $\boldsymbol{\varphi}, \boldsymbol{\psi} \in H^{1}(\Omega)^{2} \times H^{1}(\Omega)^{2}$

$$
a(\boldsymbol{\varphi}, \boldsymbol{\psi})=\int_{\Omega}\left(\nabla \varphi_{P}+\operatorname{curl} \varphi_{S}\right) \cdot\left(\nabla \psi_{P}+\operatorname{curl} \psi_{S}\right) d \boldsymbol{x} .
$$

Proof. Let us denote $\tilde{a}(\boldsymbol{\varphi}, \boldsymbol{\psi})$ the right hand side of $(24)$. We obtain after expansion, using $\operatorname{curl} \varphi_{S} \cdot \operatorname{curl} \psi_{S}=\nabla \varphi_{S} \cdot \nabla \psi_{S}$ and $(22)$

$$
\tilde{a}(\boldsymbol{\varphi}, \boldsymbol{\psi})=a_{\Omega}(\boldsymbol{\varphi}, \boldsymbol{\psi})+\int_{\Omega} \nabla \varphi_{P} \cdot \operatorname{curl} \psi_{S} \mathrm{~d} \boldsymbol{x}+\int_{\Omega} \nabla \psi_{P} \cdot \operatorname{curl} \varphi_{S} \mathrm{~d} \boldsymbol{x}
$$

Next we observe that (Green's formula and div curl $=0$ )

$$
\int_{\Omega} \nabla \psi_{P} \cdot \operatorname{curl} \varphi_{S} \mathrm{~d} \boldsymbol{x}=\int_{\Gamma} \psi_{P} \operatorname{curl} \varphi_{S} \cdot \boldsymbol{n} \mathrm{d} \boldsymbol{\gamma}=-\int_{\Gamma} \psi_{P} \partial_{\boldsymbol{\tau}} \varphi_{S} \mathrm{~d} \boldsymbol{\gamma}
$$

In the same way

$$
\int_{\Omega} \nabla \varphi_{P} \cdot \operatorname{curl} \psi_{S} \mathrm{~d} \boldsymbol{x}=-\int_{\Gamma} \varphi_{P} \partial_{\boldsymbol{\tau}} \psi_{S} \mathrm{~d} \boldsymbol{\gamma}=\int_{\Gamma} \partial_{\boldsymbol{\tau}} \varphi_{P} \psi_{S} \mathrm{~d} \boldsymbol{\gamma}
$$

after integration by parts along the boundary. To conclude we add (25) and (26) to infer that by definition of $a_{\Gamma}(\cdot, \cdot)$ (see (23))

$$
\tilde{a}(\boldsymbol{\varphi}, \boldsymbol{\psi})=a_{\Omega}(\boldsymbol{\varphi}, \boldsymbol{\psi})+a_{\Gamma}(\boldsymbol{\varphi}, \boldsymbol{\psi})=a(\boldsymbol{\varphi}, \boldsymbol{\psi})
$$

according to the definition 21 .

This lemma proves that the bilinear form $a(\cdot, \cdot)$ is positive, but also suggests that $H^{1}(\Omega)^{2}$ is not the appropriate variational space for the weak formulation because of the coercivity requirement. That is why we introduce the space

$$
\boldsymbol{V}:=\left\{\boldsymbol{\varphi}=\left(\varphi_{P}, \varphi_{S}\right) \in L^{2}(\Omega)^{2} \text { such that } \nabla \varphi_{P}+\operatorname{curl} \varphi_{S} \in L^{2}(\Omega)^{2}\right\} .
$$

Interpreting $\varphi$ as a vector field whose first component is $\varphi_{P}$ and its second component is $\varphi_{S}$, one notices that

$$
\nabla \varphi_{P}+\operatorname{curl} \varphi_{S}=\left(\begin{array}{c}
\operatorname{div} \varphi \\
-\operatorname{curl} \varphi
\end{array}\right)
$$


in such a way that the space $\boldsymbol{V}$ can be alternatively characterized as

$$
\boldsymbol{V}=H(\operatorname{div}, \Omega) \cap H(\operatorname{curl}, \Omega),
$$

a well known space from the theory of $\mathrm{M}$

axwell's equations [10, 12]. This space striclty contains $H^{1}(\Omega)^{2}$ (see also Remark 2.2. . However it is known [13] that

$$
\text { the space } \left.\mathcal{D}(\bar{\Omega})^{2} \text { (and thus, the space } H^{1}(\Omega)^{2}\right) \text { is dense in } \boldsymbol{V} \text {. }
$$

Lemma 2.1 allows us to extend continuously the bilinear form $a(\cdot, \cdot)$ to $\boldsymbol{V}$

$$
\forall(\boldsymbol{\varphi}, \boldsymbol{\psi}) \in \boldsymbol{V} \times \boldsymbol{V}, \quad a(\boldsymbol{\varphi}, \boldsymbol{\psi})=\int_{\Omega}\left(\nabla \varphi_{P}+\operatorname{curl} \varphi_{S}\right) \cdot\left(\nabla \psi_{P}+\operatorname{curl} \psi_{S}\right) \mathrm{d} \boldsymbol{x},
$$

the expression 21 being only valid when $(\boldsymbol{\varphi}, \boldsymbol{\psi}) \in H^{1}(\Omega)^{2} \times H^{1}(\Omega)^{2}$. Note that thanks to $(28)$ an alternative formula for the bilinear form $a(\cdot, \cdot)$ is

$$
\forall(\boldsymbol{\varphi}, \boldsymbol{\psi}) \in \boldsymbol{V} \times \boldsymbol{V}, \quad a(\boldsymbol{\varphi}, \boldsymbol{\psi})=\int_{\Omega}(\operatorname{div} \boldsymbol{\varphi} \operatorname{div} \boldsymbol{\psi}+\operatorname{curl} \boldsymbol{\varphi} \operatorname{curl} \boldsymbol{\psi}) \mathrm{d} \boldsymbol{x} .
$$

Finally, the correct variational formulation of our problem is

$$
\left\{\begin{array}{l}
\text { Find } \boldsymbol{\varphi}(t): \mathbb{R}^{+} \longrightarrow \boldsymbol{V} \text { such that }\left(\boldsymbol{\varphi}, \partial_{t} \boldsymbol{\varphi}\right)(t=0)=(\mathbf{0}, \mathbf{0}) \text { and } \\
\frac{\mathrm{d}^{2}}{\mathrm{~d} t^{2}} m_{\Omega}(\boldsymbol{\varphi}(t), \boldsymbol{\psi})+a(\boldsymbol{\varphi}(t), \boldsymbol{\psi})=l(t, \boldsymbol{\psi}), \quad \forall \boldsymbol{\psi} \in \boldsymbol{V} .
\end{array}\right.
$$

Remark 2.2. It is worthwhile emphasizing that functions in $H^{1}(\Omega)^{2}$ and $\boldsymbol{V}$ only differ close to the boundary; each function in $\boldsymbol{V}$ has $H^{1}(\omega)^{2}$-regularity for any open set $\omega$ such that $\bar{\omega} \subset \Omega$.

Energy considerations. As it is classical, the energy naturally associated with (33), which is conserved as soon as the right hand side vanishes, is

$$
E_{D}(t)=\frac{1}{2}\left[m_{\Omega}\left(\partial_{t} \varphi(t), \partial_{t} \varphi(t)\right)+a(\varphi(t), \varphi(t))\right] .
$$

Equivalently it can be written as

$$
E_{D}(t)=\frac{1}{2 V_{P}^{2}} \int_{\Omega}\left|\partial_{t} \varphi_{P}\right|^{2} \mathrm{~d} \boldsymbol{x}+\frac{1}{2 V_{S}^{2}} \int_{\Omega}\left|\partial_{t} \varphi_{S}\right|^{2} \mathrm{~d} \boldsymbol{x}+\frac{1}{2} \int_{\Omega}\left|\nabla \varphi_{P}+\operatorname{curl} \varphi_{S}\right|^{2} \mathrm{~d} \boldsymbol{x} .
$$

The first remark is that $E_{D}(t)$ differs from $E_{P}(t)+E_{S}(t)$ where $E_{P}(t)$ and $E_{S}(t)$ are the energies usually associated to each of the wave equation $(12)$, namely

$$
E_{Q}(t)=\frac{1}{2 V_{Q}^{2}} \int_{\Omega}\left|\partial_{t} \varphi_{Q}\right|^{2} \mathrm{~d} \boldsymbol{x}+\frac{1}{2} \int_{\Omega}\left|\nabla \varphi_{Q}\right|^{2} \mathrm{~d} \boldsymbol{x}, \quad Q \in\{P, S\} .
$$


More precisely, the two quantities differ by a boundary term since, assuming that $\varphi_{P}$ and $\varphi_{S}$ are smooth enough, one computes that

$$
E_{D}(t)=E_{P}(t)+E_{S}(t)+\frac{1}{2} \int_{\Gamma}\left(\partial_{\boldsymbol{\tau}} \varphi_{P} \varphi_{S}-\varphi_{P} \partial_{\boldsymbol{\tau}} \varphi_{S}\right) \mathrm{d} \boldsymbol{\gamma} .
$$

It is also interesting (and this will be even important for the Neumann problem) to relate this energy to the classical elastic energy

$$
E_{e l}(t)=E_{c}(t)+E_{p}(t),
$$

where the kinetic and potential energies $E_{c}(t)$ and $E_{p}(t)$ are given by

$$
E_{c}(t)=\frac{\rho}{2} \int_{\Omega}|\boldsymbol{v}|^{2} \mathrm{~d} \boldsymbol{x}, \quad E_{p}(t)=\frac{1}{2} \int_{\Omega} \underline{\boldsymbol{\sigma}}(\boldsymbol{u}): \underline{\boldsymbol{\varepsilon}}(\boldsymbol{u}) \mathrm{d} \boldsymbol{x},
$$

where $\underline{\boldsymbol{\sigma}}(\boldsymbol{u})=\lambda \operatorname{div} \boldsymbol{u} \underline{\boldsymbol{I}}+2 \mu \underline{\boldsymbol{\varepsilon}}(\boldsymbol{u})$ and, using Einstein's convention,

$$
\underline{\sigma}: \underline{\varepsilon}=\sigma_{i j} \varepsilon_{i j}
$$

is the tensor product between two tensors. Using (11) we see that, in the absence of source term

$$
E_{c}(t)=\frac{\rho}{2} \int_{\Omega}\left|\nabla \varphi_{P}+\operatorname{curl} \varphi_{S}\right|^{2} \mathrm{~d} \boldsymbol{x}=\frac{\rho}{2} a(\boldsymbol{\varphi}, \boldsymbol{\varphi}) .
$$

On the other hand, thanks to Hooke's law (3)

$$
E_{p}(t)=\frac{\lambda}{2} \int_{\Omega}|\operatorname{div} \boldsymbol{u}|^{2} \mathrm{~d} \boldsymbol{x}+\mu \int_{\Omega}|\underline{\varepsilon}(\boldsymbol{u})|^{2} \mathrm{~d} \boldsymbol{x} .
$$

Next we use the following lemma which is closely related to the first Korn's inequality and whose proof will be provided below for completeness.

Lemma 2.3. For all $\boldsymbol{u} \in H^{1}(\Omega)^{2}$ one has

$$
\int_{\Omega}|\underline{\varepsilon}(\boldsymbol{u})|^{2} d \boldsymbol{x}=\int_{\Omega}|\operatorname{div} \boldsymbol{u}|^{2} d \boldsymbol{x}+\frac{1}{2} \int_{\Omega}|\operatorname{curl} \boldsymbol{u}|^{2} d \boldsymbol{x}-2 \int_{\Gamma} u_{2} \partial_{\boldsymbol{\tau}} u_{1} d \boldsymbol{\gamma} .
$$

Proof. It is based on the following algebraic manipulations :

$$
\begin{aligned}
|\underline{\varepsilon}(\boldsymbol{u})|^{2} & =\left|\partial_{1} u_{1}\right|^{2}+\left|\partial_{2} u_{2}\right|^{2}+\frac{1}{2}\left|\partial_{1} u_{2}+\partial_{2} u_{1}\right|^{2} \\
& =\left|\partial_{1} u_{1}+\partial_{2} u_{2}\right|^{2}-2 \partial_{1} u_{1} \partial_{2} u_{2}+\frac{1}{2}\left|\partial_{1} u_{2}-\partial_{2} u_{1}\right|^{2}+2 \partial_{1} u_{2} \partial_{2} u_{1} \\
& =|\operatorname{div} \boldsymbol{u}|^{2}+\frac{1}{2}|\operatorname{curl} \boldsymbol{u}|^{2}+2 \operatorname{curl} u_{1} \cdot \nabla u_{2}
\end{aligned}
$$

One obtains 38 by integration of the above equality over $\Omega$ since, by an integration by parts

$$
\int_{\Omega} \operatorname{curl} u_{1} \cdot \nabla u_{2} \mathrm{~d} \boldsymbol{x}=-\int_{\Gamma} u_{2} \partial_{\boldsymbol{\tau}} u_{1} \mathrm{~d} \boldsymbol{\gamma}
$$


We can use this lemma to rewrite the potential energy as

$$
E_{p}(t)=\frac{\lambda+2 \mu}{2} \int_{\Omega}|\operatorname{div} \boldsymbol{u}|^{2} \mathrm{~d} \boldsymbol{x}+\frac{\mu}{2} \int_{\Omega}|\operatorname{curl} \boldsymbol{u}|^{2} \mathrm{~d} \boldsymbol{x}-2 \mu \int_{\Gamma} u_{2} \partial_{\boldsymbol{\tau}} u_{1} \mathrm{~d} \boldsymbol{\gamma}
$$

Then considering the Dirichlet boundary condition and using definition of potentials (8) one gets

$$
E_{p}(t)=\frac{\rho}{2} m_{\Omega}\left(\partial_{t} \varphi, \partial_{t} \varphi\right) .
$$

Finally, joining (36) and 40 we observe that the elastic energy is related to $E_{D}(t)$ as follows

$$
E_{e l}(t)=\rho E_{D}(t)
$$

Finite element approximation. The space discretization of $(33)$ relies on the construction of a finite dimensional approximation of the space $\boldsymbol{V}$ that will be denoted by $\boldsymbol{V}_{h}$. Owing to Remark 2.2, such $\boldsymbol{V}_{h}$ will be naturally sought in the form

$$
\boldsymbol{V}_{h}=V_{P, h} \times V_{S, h},
$$

where $V_{P, h}$ and $V_{S, h}$ are standard Lagrange finite element approximations for $H^{1}(\Omega)$ (see for example [14]), the density result in $(30)$ ensuring that appropriate approximation properties of $\boldsymbol{V}$ by $\boldsymbol{V}_{h}$ are preserved. However the important property is that the spaces $V_{P, h}$ and $V_{S, h}$ can be constructed in different meshes and that is why this method gives us the flexibility for adapting each space discretization to each type of wave. The semi-discrete problem writes

$$
\left\{\begin{array}{l}
\text { Find } \boldsymbol{\varphi}_{h}(t): \mathbb{R}^{+} \longrightarrow \boldsymbol{V}_{h} \text { such that }\left(\boldsymbol{\varphi}_{h}, \partial_{t} \boldsymbol{\varphi}_{h}\right)(t=0)=(\mathbf{0}, \mathbf{0}) \text { and } \\
\frac{\mathrm{d}^{2}}{\mathrm{~d} t^{2}} m_{\Omega}\left(\boldsymbol{\varphi}_{h}(t), \boldsymbol{\psi}_{h}\right)+a\left(\boldsymbol{\varphi}_{h}(t), \boldsymbol{\psi}_{h}\right)=l\left(t, \boldsymbol{\psi}_{h}\right), \quad \forall \boldsymbol{\psi}_{h} \in \boldsymbol{V}_{h} .
\end{array}\right.
$$

In practice, the embedding $\boldsymbol{V}_{h} \subset H^{1}(\Omega)^{2}$ authorizes us to use the formula (21) to evaluate $a(\cdot, \cdot)$ and that is why after decomposition of the discrete unknowns $\left(\varphi_{P, h}, \varphi_{S, h}\right)$ in the respective Lagrange elements, the formulation takes the following form

$$
\mathbb{M}_{h}^{\Omega} \frac{\mathrm{d}^{2} \boldsymbol{\Phi}_{h}}{\mathrm{~d} t^{2}}+\mathbb{A}_{h}^{\Omega} \boldsymbol{\Phi}_{h}+\mathbb{A}_{h}^{\Gamma} \boldsymbol{\Phi}_{h}=\boldsymbol{F}_{h}
$$

where $\boldsymbol{\Phi}_{h}^{t}=\left(\boldsymbol{\Phi}_{P, h}, \boldsymbol{\Phi}_{S, h}\right)^{t}$ are the vectors of the Lagrange degrees of freedom of $\varphi_{P, h}$ and $\varphi_{S, h}$ and whose dimensions are not necessarily the same. The symmetric matrices $\mathbb{M}_{h}^{\Omega}$ and $\mathbb{A}_{h}^{\Omega}$ are block diagonal

$$
\mathbb{M}_{h}^{\Omega}=\left(\begin{array}{cc}
\mathbb{M}_{P, h}^{\Omega} & \mathbb{O} \\
\mathbb{O} & \mathbb{M}_{S, h}^{\Omega}
\end{array}\right), \quad \mathbb{A}_{h}^{\Omega}=\left(\begin{array}{cc}
\mathbb{A}_{P, h}^{\Omega} & \mathbb{O} \\
\mathbb{O} & \mathbb{A}_{S, h}^{\Omega}
\end{array}\right)
$$

while the coupling matrix $\mathbb{A}_{h}^{\Gamma}$ has the following structure

$$
\mathbb{A}_{h}^{\Gamma}=\left(\begin{array}{cc}
\mathbb{O} & \mathbb{C}_{h}^{\Gamma} \\
\left(\mathbb{C}_{h}^{\Gamma}\right)^{t} & \mathbb{O}
\end{array}\right)
$$


where the matrix $\mathbb{C}_{h}^{\Gamma}$ is very sparse since it only couples neighboring degrees of freedom that are located along the boundary. Moreover, for efficiency considerations, we shall assume that integrals are computed by specific quadrature formulas that achieve mass lumping, that is to say that $\mathbb{M}_{h}^{\Omega}$ is diagonal. Many techniques can be used to achieve this goal (see [15], 16], 17] and the references therein).

Time discretization. In [6], our goal was to propose a scheme that should be as close as possible to standard explicit leap-frog discretization of each wave equation, one constraint being that the coupling terms should not have any influence on the resulting CFL stability condition. This led us to propose a semi-implicit method consisting in treating in a explicit way the terms associated to the volumic terms and implicitly those related to the coupling through the boundary (using the so called trapezoidal rule). This provided the following scheme we show below. Let us consider a constant time discretization step $\Delta t$ and let us denote by $\varphi_{h}^{n}$ the approximation of $\varphi_{h}(n \Delta t)$. The total discretization of the problem reads as follows

$$
\left\{\begin{array}{l}
\text { Find } \boldsymbol{\varphi}_{h}^{n+1} \in \boldsymbol{V}_{h} \text { such that for } n \geq 1 \\
m_{\Omega}\left(\frac{\boldsymbol{\varphi}_{h}^{n+1}-2 \boldsymbol{\varphi}_{h}^{n}+\boldsymbol{\varphi}_{h}^{n-1}}{\Delta t^{2}}, \boldsymbol{\psi}_{h}\right)+a_{\Omega}\left(\boldsymbol{\varphi}_{h}^{n}, \boldsymbol{\psi}_{h}\right)+ \\
a_{\Gamma}\left(\frac{\boldsymbol{\varphi}_{h}^{n+1}+2 \boldsymbol{\varphi}_{h}^{n}+\boldsymbol{\varphi}_{h}^{n-1}}{4}, \boldsymbol{\psi}_{h}\right)=l\left(t^{n}, \boldsymbol{\psi}_{h}\right), \quad \forall \boldsymbol{\psi}_{h} \in \boldsymbol{V}_{h},
\end{array}\right.
$$

where $\left(\varphi_{h}^{0}, \varphi_{h}^{1}\right)$, the approximations of $(\varphi(t=0), \boldsymbol{\varphi}(t=\Delta t))$ are assumed to vanish. Its algebraic representation is given by

$$
\mathbb{M}_{h}^{\Omega} \frac{\boldsymbol{\Phi}_{h}^{n+1}-2 \boldsymbol{\Phi}_{h}^{n}+\boldsymbol{\Phi}_{h}^{n-1}}{\Delta t^{2}}+\mathbb{A}_{h}^{\Omega} \boldsymbol{\Phi}_{h}^{n}+\mathbb{A}_{h}^{\Gamma} \frac{\boldsymbol{\Phi}_{h}^{n+1}+2 \boldsymbol{\Phi}_{h}^{n}+\boldsymbol{\Phi}_{h}^{n-1}}{4}=\boldsymbol{F}_{h}^{n},
$$

that is a discrete version of (33). The stability analysis of (44) relies on discrete energy identity which can be reduced by linearity to the case of a zero right hand side (we omit here the details which are classical). The main lemma is the following

Lemma 2.4. Assuming $l\left(t^{n}, \psi_{h}\right)=0$ for $n \geq n^{*}$, any solution of (44) satisfies

$$
\forall n \geq n^{*}, \quad E_{h}^{n+\frac{1}{2}}=E_{h}^{\frac{1}{2}},
$$

where the discrete energy $E_{h}^{n+\frac{1}{2}}$ is defined by

$$
\begin{aligned}
E_{h}^{n+\frac{1}{2}}:= & \frac{1}{2}\left(m_{\Omega}-\frac{\Delta t^{2}}{4} a_{\Omega}\right)\left(\frac{\varphi_{h}^{n+1}-\varphi_{h}^{n}}{\Delta t}, \frac{\varphi_{h}^{n+1}-\varphi_{h}^{n}}{\Delta t}\right)+ \\
& \frac{1}{2} a\left(\frac{\varphi_{h}^{n+1}+\varphi_{h}^{n}}{2}, \frac{\varphi_{h}^{n+1}+\varphi_{h}^{n}}{2}\right) .
\end{aligned}
$$


Proof. (Sketch) The approach is classical. The key idea is to write

$$
\varphi_{h}^{n}=\frac{\varphi_{h}^{n+1}+2 \varphi_{h}^{n}+\varphi_{h}^{n-1}}{4}-\frac{\Delta t^{2}}{4} \frac{\varphi_{h}^{n+1}-2 \varphi_{h}^{n}+\varphi_{h}^{n-1}}{\Delta t^{2}}
$$

inside $a_{\Omega}\left(\boldsymbol{\varphi}_{h}^{n}, \boldsymbol{\psi}_{h}\right)$ to rewrite 44 as a modification of the fully implicit scheme

$$
\begin{aligned}
& \left(m_{\Omega}-\frac{\Delta t^{2}}{4} a_{\Omega}\right)\left(\frac{\boldsymbol{\varphi}_{h}^{n+1}-2 \boldsymbol{\varphi}_{h}^{n}+\boldsymbol{\varphi}_{h}^{n-1}}{\Delta t^{2}}, \boldsymbol{\psi}_{h}\right)+ \\
& \quad a\left(\frac{\boldsymbol{\varphi}_{h}^{n+1}+2 \boldsymbol{\varphi}_{h}^{n}+\boldsymbol{\varphi}_{h}^{n-1}}{4}, \boldsymbol{\psi}_{h}\right)=0, \quad \forall \boldsymbol{\psi}_{h} \in \boldsymbol{V}_{h} .
\end{aligned}
$$

Then it suffices to take

$$
\psi_{h}=\frac{\varphi_{h}^{n+1}-\varphi_{h}^{n-1}}{2 \Delta t}
$$

into the formulation and apply usual manipulations about differences of squares to obtain (the symmetry of the various bilinear forms is fundamental)

$$
\frac{1}{2 \Delta t}\left[E_{h}^{n+\frac{1}{2}}-E_{h}^{n-\frac{1}{2}}\right]=0,
$$

which leads to the result.

Using this lemma we can write now the main stability theorem for which we need to introduce the quantities

$$
c_{Q}(h)=\max _{\varphi_{Q, h} \in V_{Q, h}} \frac{a_{Q}\left(\varphi_{Q, h}, \varphi_{Q, h}\right)}{m_{Q}\left(\varphi_{Q, h}, \varphi_{Q, h}\right)}, Q \in\{P, S\} .
$$

Theorem 2.5. The numerical scheme (44) is well-posed and stable under the CFL stability condition

$$
\frac{\Delta t^{2}}{4} \max \left[c_{P}(h), c_{S}(h)\right] \leq 1
$$

Proof. (Sketch) As it is classical, the stability follows from the positivity of the discrete energy. Since the quadratic form $a(\cdot, \cdot)$ is positive (see Lemma2.1), this reduces to the positivity of the quadratic form associated to

$$
\left(m_{\Omega}-\frac{\Delta t^{2}}{4} a_{\Omega}\right)(\cdot, \cdot)
$$

over the space $V_{h}$, that is to say (see $(20)$ and $(22)$ ), to the positivity of the quadratic form associated to

$$
\left(m_{P}-\frac{\Delta t^{2}}{4} a_{P}\right)(\cdot, \cdot) \text { and }\left(m_{S}-\frac{\Delta t^{2}}{4} a_{S}\right)(\cdot, \cdot)
$$

over the spaces $V_{P, h}$ and $V_{S, h}$. It is then easy to conclude. 
Remark 2.6. It can be shown that the condition (48) is also a necessary stability condition. Indeed for sources with compact support, for a certain time we simply solve two decoupled scalar wave equations (the boundary conditions do not matter) and we need to satisfy the CFL condition associated to each corresponding scheme which is nothing but (48).

It is worthwhile to make the following comments:

- Considering the particular example of $\mathbb{P}_{k}$ or $\mathbb{Q}_{k}$ finite elements and uniform meshes of respective sizes $h_{P}$ and $h_{S}$, it is well-known that, at least asymptotically, when $h_{P}$ and $h_{S}$ approaches to zero

$$
c_{P}(h) \approx C_{k}^{2} \frac{V_{P}^{2}}{h_{P}^{2}} \text { and } c_{S}(h) \approx C_{k}^{2} \frac{V_{S}^{2}}{h_{S}^{2}} .
$$

where the constant $C_{k}$ only depends on $k$. On the other hand, adapting each space step to the corresponding wavelength (that is proportional to the associated velocity) leads to choose

$$
\frac{h_{P}}{V_{P}}=\frac{h_{S}}{V_{S}} .
$$

Indeed, denoting $T_{\star}$ the typical time scale of the problem (of the source term for instance) we can define two associated wavelengths (typical space scales for $P$ and $S$ waves respectively) by

$$
\lambda_{P}=V_{P} T_{\star}, \quad \lambda_{S}=V_{S} T_{\star} .
$$

Let $N_{P}$ and $N_{S}$ the number of mesh points per P-wavelength and Swavelength respectively that is to say

$$
N_{P}:=\frac{\lambda_{P}}{h_{P}}=\frac{V_{P}}{h_{P}} T_{\star}, \quad N_{S}:=\frac{\lambda_{S}}{h_{S}}=\frac{V_{S}}{h_{S}} T_{\star},
$$

so that (50) implies $N:=N_{P}=N_{S}$. Then, consirering (49) as equalities, the stability condition (48) reduces to

$$
\frac{\Delta t^{2}}{T_{\star}^{2}} \leq \frac{4}{N^{2} C_{k}^{2}},
$$

which means that the choice of the time step is not affected by the ratio $V_{P} / V_{S}$. Thus, the global cost computed as in (1) is also independent on the ratio between $V_{P}$ and $V_{S}$. Similar conclusions can be obtained when using different polynomial degrees instead of different meshes.

- From the computational point of view one has to invert at each time step the matrix

$$
\mathbb{M}_{h}^{\Omega}+\frac{\Delta t^{2}}{4} \mathbb{A}_{h}^{\Gamma}
$$

(see (45)). Considering the sparsity pattern of $\mathbb{A}_{h}^{\Gamma}$ and that $\mathbb{M}_{h}^{\Omega}$ is diagonal (by mass lumping), the evaluation of interior degrees of freedom is completely explicit while the computation of the boundary degrees of freedom amounts to invert a sparse linear system, the invertibility of which is guaranteed by the stability theorem. 


\section{The case of a free surface boundary condition}

In this section, we consider the case of a free surface boundary condition (the corresponding problem will be referred to as the Neumann problem in the sequel), that is, written in terms of the unknow $\boldsymbol{v}$

$$
\underline{\boldsymbol{\sigma}}(\boldsymbol{v}) \boldsymbol{n} \equiv \lambda \operatorname{div} \boldsymbol{v} \boldsymbol{n}+2 \mu \underline{\boldsymbol{\varepsilon}}(\boldsymbol{v}) \boldsymbol{n}=\mathbf{0}, \quad \text { on } \Gamma .
$$

In Section 3.1 we will recap some preliminary results on the Neumann problem. As it will be shown in Section 3.2, a naive extension of the technique explained in the previous section leads to an unstable variational formulation. The functional space in which it is set appears to be too large. To overcome this problem, in Section 3.3 this space is constrained in such a way that the new formulation is stabilized and suitable for finite element approximations.

For the sake of simplicity (the reader will easily convince himself that this is not restrictive), we shall assume that $\Omega$ is bounded and simply connected, thus that $\Gamma$ is a closed curve (see Figure 1). We shall also assume that $\Gamma$ is parameterized by $\boldsymbol{x}(s) \in W^{1, \infty}(0, L)$ where $s$ is the curvilinear abscissa along $\Gamma$ and $L$ is the total length of $\Gamma$. Finally, we shall use the following notation for denoting a particular primitive of a function defined on $\Gamma$ (where we arbitrarily particularize the point associated to $s=0$ but this choice has no influence)

$$
\forall \eta \in L^{2}(\Gamma), \quad \mathcal{I} \eta(s):=\int_{0}^{s} \eta(\sigma) \mathrm{d} \sigma \in H^{1}(\Gamma) .
$$

It is clear that $\mathcal{I}$ can be extended as a linear continuous operator

$$
\mathcal{I} \in \mathcal{L}\left(H^{-1 / 2}(\Gamma), H^{1 / 2}(\Gamma)\right)
$$

\subsection{Preliminary recaps on the Neumann problem}

In this section we are interested in the problem

$$
\begin{cases}\rho \partial_{t}^{2} \boldsymbol{u}-\operatorname{div} \underline{\boldsymbol{\sigma}}(\boldsymbol{u})=\boldsymbol{f}, & \text { in } \Omega, \\ \underline{\boldsymbol{\sigma}}(\boldsymbol{u}) \boldsymbol{n}=\mathbf{0}, & \text { on } \Gamma,\end{cases}
$$

completed with the initial conditions (7). In this problem, a particular role is played by the 3 dimensional space of the so-called rigid displacements

$$
\begin{aligned}
\boldsymbol{R}(\Omega) & =\left\{\boldsymbol{w}_{R} \in L^{2}(\Omega)^{2} / \underline{\boldsymbol{\varepsilon}}\left(\boldsymbol{w}_{R}\right)=\underline{\mathbf{0}}\right\} \\
& =\left\{a\left(x_{2},-x_{1}\right)^{t}+\left(b_{1}, b_{2}\right)^{t},\left(a,\left(b_{1}, b_{2}\right)\right) \in \mathbb{R} \times \mathbb{R}^{2}\right\}
\end{aligned}
$$

We introduce the spaces

$$
\begin{aligned}
\boldsymbol{L}_{R}^{2}(\Omega) & =\left\{\boldsymbol{w} \in L^{2}(\Omega)^{2} / \int_{\Omega} \boldsymbol{w} \cdot \boldsymbol{w}_{R} \mathrm{~d} \boldsymbol{x}=0, \forall \boldsymbol{w}_{R} \in \boldsymbol{R}(\Omega)\right\} \\
\boldsymbol{H}_{R}^{1}(\Omega) & =H^{1}(\Omega)^{2} \cap \boldsymbol{L}_{R}^{2}(\Omega) .
\end{aligned}
$$


so that we have the direct sums

$$
L^{2}(\Omega)^{2}=\boldsymbol{L}_{R}^{2}(\Omega) \oplus \boldsymbol{R}(\Omega), \quad H^{1}(\Omega)^{2}=\boldsymbol{H}_{R}^{1}(\Omega) \oplus \boldsymbol{R}(\Omega),
$$

which are orthogonal in $L^{2}(\Omega)^{2}$. A classical but important property of problem (54) is provided in the following lemma.

Lemma 3.1. If $\boldsymbol{f}(\cdot, t) \in \boldsymbol{L}_{R}^{2}(\Omega), \forall t \geq 0$, then

$$
\forall t \geq 0, \quad \boldsymbol{u}(\cdot, t) \in \boldsymbol{H}_{R}^{1}(\Omega) .
$$

Proof. Multiply $(54)$ by $\boldsymbol{w}_{R}$ and integrate over $\Omega$. Using Green's formula,

$$
\frac{\mathrm{d}^{2}}{\mathrm{~d} t^{2}}\left(\rho \int_{\Omega} \boldsymbol{u}(\cdot, t) \cdot \boldsymbol{w}_{R} \mathrm{~d} \boldsymbol{x}\right)=0 \quad \forall \boldsymbol{w}_{R} \in \boldsymbol{R}(\Omega) .
$$

One concludes using the initial conditions.

In the sequel of this section we restrict ourselves to source terms satisfying

$$
\boldsymbol{f}(\cdot, t) \in \boldsymbol{L}_{R}^{2}(\Omega), \forall t \geq 0
$$

This is not restrictive due to the following remark.

Remark 3.2. For a general source term $\boldsymbol{f}=\boldsymbol{f}_{R}+\boldsymbol{f}_{R}^{\perp}$, with $\boldsymbol{f}_{R}(\cdot, t) \in \boldsymbol{L}_{R}^{2}(\Omega)$ and $\boldsymbol{f}_{R}^{\perp}(\cdot, t) \in \boldsymbol{R}(\Omega)$, it is easy to see that the solution $\boldsymbol{u}$ of $(54)$ can be decomposed as $\boldsymbol{u}=\boldsymbol{u}_{R}+\boldsymbol{u}_{R}^{\perp}$, where $\boldsymbol{u}_{R}$ is the solution of (54) with source term given by $\boldsymbol{f}_{R}$ and $\boldsymbol{u}_{R}^{\perp}$ is given by

$$
\boldsymbol{u}_{R}^{\perp}=\frac{1}{\rho} \int_{0}^{t}(t-s) \boldsymbol{f}_{R}^{\perp}(\cdot, s) \mathrm{d} s .
$$

Another important property is Korn's inequality in $\boldsymbol{H}_{R}^{1}(\Omega)$ (see [18]):

Proposition 3.3. There exists a constant $\mathcal{C}_{\Omega}>0$ such that

$$
\forall \boldsymbol{w} \in \boldsymbol{H}_{R}^{1}(\Omega), \quad\|\boldsymbol{w}\|_{H^{1}(\Omega)}^{2} \leq \mathcal{C}_{\Omega} \int_{\Omega}|\underline{\underline{\varepsilon}}(\boldsymbol{w})|^{2} \mathrm{~d} \boldsymbol{x} .
$$

\subsection{The naive approach. Stability issues}

\subsubsection{The free boundary condition with potentials}

Of course, the first step consists in rewriting the free boundary condition (51) in terms of the potentials defined by (8) and (10) (as we did in section 2.2 by transforming the Dirichlet condition (15) into (17)). However, from (11), we see that, a priori, the condition (51) leads to an equation involving the second order space derivatives of the potentials, thus not well adapted for a finite element formulation. To overcome this, let us assume for a while that we know the value $\boldsymbol{v}_{\Gamma}$ of the velocity field on the boundary, or, in other words, that we want 
to treat a non homogeneous Dirichlet boundary condition with $\boldsymbol{v}=\boldsymbol{v}_{\Gamma}$ on the boundary. Then, proceeding as in section 2.2 for obtaining (17), we would use the non homogeneous boundary condition to obtain

$$
\partial_{\boldsymbol{n}} \varphi_{P}=\partial_{\boldsymbol{\tau}} \varphi_{S}-\boldsymbol{g} \cdot \boldsymbol{n}+\boldsymbol{v}_{\Gamma} \cdot \boldsymbol{n}, \quad \partial_{\boldsymbol{n}} \varphi_{S}=-\partial_{\boldsymbol{\tau}} \varphi_{P}-\boldsymbol{g} \cdot \boldsymbol{\tau}+\boldsymbol{v}_{\Gamma} \cdot \boldsymbol{\tau} .
$$

Then, since we do not know $\boldsymbol{v}_{\Gamma}$, we would like to compute it as a function of $\boldsymbol{\varphi}$ using the free boundary condition (51). To do so, we first remark that

$$
\underline{\boldsymbol{\varepsilon}}(\boldsymbol{v})=2 \operatorname{div} \boldsymbol{v} \underline{\boldsymbol{I}}-\operatorname{curl} \boldsymbol{v} \underline{\boldsymbol{J}}+\underline{\boldsymbol{H}}(\boldsymbol{v}),
$$

where $\quad \underline{\boldsymbol{J}}=\left(\begin{array}{cc}0 & 1 \\ -1 & 0\end{array}\right), \quad \underline{\boldsymbol{H}}(\boldsymbol{v})=\left(\begin{array}{cc}-\partial_{2} v_{2} & \partial_{1} v_{2} \\ \partial_{2} v_{1} & -\partial_{1} v_{1}\end{array}\right)$,

so that the free boundary condition (51) can be rewritten

$$
(\lambda+2 \mu) \operatorname{div} \boldsymbol{v} \boldsymbol{n}-\mu \operatorname{curl} \boldsymbol{v} \boldsymbol{\tau}+2 \mu \underline{\boldsymbol{H}}(\boldsymbol{v}) \boldsymbol{n}=0, \quad \text { on } \Gamma .
$$

We deduce from $(8)$, differentiating in time, that

$$
\rho \partial_{t}^{2} \varphi_{P}=(\lambda+2 \mu) \operatorname{div} \boldsymbol{v}, \quad \rho \partial_{t}^{2} \varphi_{S}=-\mu \operatorname{curl} \boldsymbol{v}, \quad \text { in } \Omega,
$$

so that, assuming sufficient smoothness, we have on the boundary

$$
(\lambda+2 \mu) \operatorname{div} \boldsymbol{v} \boldsymbol{n}-\mu \operatorname{curl} \boldsymbol{v} \boldsymbol{\tau}=\rho \partial_{t}^{2} \varphi_{P} \boldsymbol{n}+\rho \partial_{t}^{2} \varphi_{S} \boldsymbol{\tau}, \quad \text { on } \Gamma .
$$

After projection on coordinate axes, using $\boldsymbol{\tau}=\left(n_{2},-n_{1}\right)$, this can be rewritten

$$
(\lambda+2 \mu) \operatorname{div} \boldsymbol{v} \boldsymbol{n}-\mu \operatorname{curl} \boldsymbol{v} \boldsymbol{n}=\rho\left(\begin{array}{c}
\partial_{t}^{2} \varphi_{P} n_{1}+\partial_{t}^{2} \varphi_{S} n_{2} \\
\partial_{t}^{2} \varphi_{P} \tau_{1}+\partial_{t}^{2} \varphi_{S} \tau_{2}
\end{array}\right) \equiv \rho\left(\begin{array}{c}
\partial_{t}^{2} \boldsymbol{\varphi} \cdot \boldsymbol{n} \\
\partial_{t}^{2} \boldsymbol{\varphi} \cdot \boldsymbol{\tau}
\end{array}\right) .
$$

In the same way, one computes that

$$
\left.\underline{\boldsymbol{H}}(\boldsymbol{v}) \boldsymbol{n}\right|_{\Gamma}=\left(\begin{array}{c}
\left.\partial_{\tau} v_{2}\right|_{\Gamma} \\
-\left.\partial_{\tau} v_{1}\right|_{\Gamma}
\end{array}\right) \equiv\left(\begin{array}{c}
\partial_{\tau} v_{\Gamma, 2} \\
-\partial_{\tau} v_{\Gamma, 1}
\end{array}\right) \quad \text { on } \Gamma .
$$

Thus, recalling that $\mu=\rho V_{S}^{2}$, the boundary condition 65 can be rewritten as

$$
\partial_{\tau} v_{\Gamma, 1}=\frac{1}{2 V_{S}^{2}} \partial_{t}^{2} \boldsymbol{\varphi} \cdot \boldsymbol{\tau}, \quad \partial_{\tau} v_{\Gamma, 2}=-\frac{1}{2 V_{S}^{2}} \partial_{t}^{2} \boldsymbol{\varphi} \cdot \boldsymbol{n}, \quad \text { on } \Gamma .
$$

This allows us, as desired, to compute $\boldsymbol{v}_{\Gamma}$ in terms of $\left(\varphi_{P}, \varphi_{S}\right)$, up to an additive constant, using the operator $\mathcal{I}$ (see (52)). More precisely, $\mathbb{P}_{0}(\Gamma)$ denoting the space of constant functions on $\Gamma$, using the fact that $\Gamma$ is a closed curve and the initial conditions (14), equations 67 are easily seen to be equivalent to

$$
\left\{\begin{array}{rlrl}
v_{\Gamma, 2}+\frac{1}{2 V_{S}^{2}} \mathcal{I}\left(\partial_{t}^{2} \boldsymbol{\varphi} \cdot \boldsymbol{n}\right) & \in \mathbb{P}_{0}(\Gamma), & & \int_{\Gamma} \boldsymbol{\varphi} \cdot \boldsymbol{n}=0 \\
v_{\Gamma, 1}-\frac{1}{2 V_{S}^{2}} \mathcal{I}\left(\partial_{t}^{2} \boldsymbol{\varphi} \cdot \boldsymbol{\tau}\right) & \in \mathbb{P}_{0}(\Gamma), & \int_{\Gamma} \boldsymbol{\varphi} \cdot \boldsymbol{\tau}=0 .
\end{array}\right.
$$


In the sequel, the last two columns of (68) will be referred to as gauge conditions.

To summarize this section, we have shown that

$\boldsymbol{v}$ satisfies $(51) \Leftrightarrow$ there exists $\left(\boldsymbol{v}_{\Gamma}, \boldsymbol{\varphi}\right)$ such that $\left.\begin{array}{|l|l|}62 & 68\end{array}\right)$ are satisfied.

The form 62,68 of the free boundary condition is the one that is useful for establishing the variational formulation of the problem (see section 3.2.2).

Remark 3.4. In more general situations where the boundary has $N_{c}>1$ connected components (each of them being smooth enough and closed) we would introduce one operator such as the one in $(52)$ per component. In consequence, the potentials should satisfy $2 N_{c}$ gauge conditions similar to those of the last column of (68).

\subsubsection{Naive variational formulation}

According to section 3.2.1 (and also section 2), the problem we want to solve is

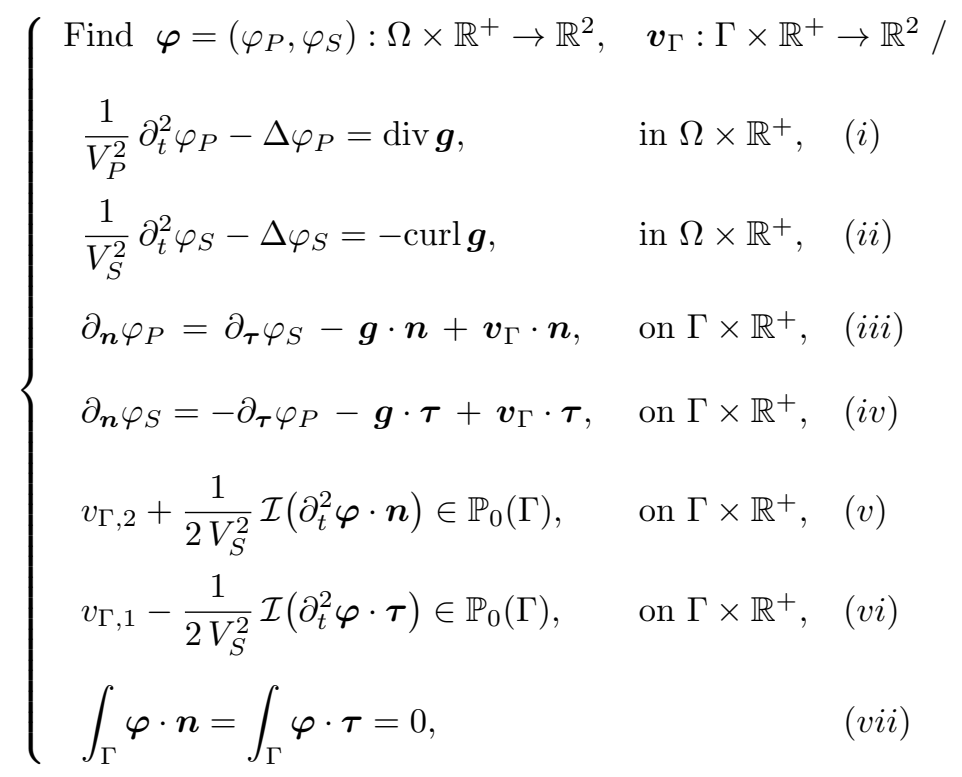

completed with the initial conditions

$$
\boldsymbol{\varphi}(\cdot, 0)=\mathbf{0}, \quad \partial_{t} \boldsymbol{\varphi}(\cdot, 0)=\mathbf{0} .
$$

We are going to provide a variational formulation of 70 , 71) which naturally eliminates $\boldsymbol{v}_{\Gamma}$ and provides a problem in $\boldsymbol{\varphi}$ only. We first take into account the last equation 70 (vii) by seeking $\varphi(\cdot, t)$ in $\boldsymbol{V}_{0}$, where $\boldsymbol{V}_{0}$ is defined by

$$
V_{0}:=\left\{\varphi \in V \text { s.t. } \int_{\Gamma} \boldsymbol{\varphi} \cdot \boldsymbol{n}=\int_{\Gamma} \boldsymbol{\varphi} \cdot \boldsymbol{\tau}=0\right\}
$$


Next, we consider test functions $\boldsymbol{\psi}=\left(\psi_{P}, \psi_{S}\right)$ in $\boldsymbol{V}_{0}$ and proceed as in Section 2.3 to obtain a variational formulation of the problem. The main difference comes from the additional boundary terms in the integration by parts due to the presence of $\boldsymbol{v}_{\Gamma}$ in the equations 770 (iii) and (iv). We obtain

$$
\frac{\mathrm{d}^{2}}{\mathrm{~d} t^{2}} m_{\Omega}(\boldsymbol{\varphi}(t), \boldsymbol{\psi})-\int_{\Gamma}\left(\boldsymbol{v}_{\Gamma} \cdot \boldsymbol{n} \psi_{P}+\boldsymbol{v}_{\Gamma} \cdot \boldsymbol{\tau} \psi_{S}\right) \mathrm{d} \sigma+a(\boldsymbol{\varphi}(t), \boldsymbol{\psi})=l(t, \boldsymbol{\psi})
$$

Next, we apply the following identity (tricky but straightforward, its verification is left to the reader)

$$
\boldsymbol{v}_{\Gamma} \cdot \boldsymbol{n} \psi_{P}+\boldsymbol{v}_{\Gamma} \cdot \boldsymbol{\tau} \psi_{S}=v_{\Gamma, 1} \boldsymbol{\psi} \cdot \boldsymbol{n}+v_{\Gamma, 2} \boldsymbol{\psi} \cdot \boldsymbol{\tau},
$$

so that, by using (70)(v) and (vi), we can eliminate $\boldsymbol{v}_{\Gamma}$ thanks to the fact that $\boldsymbol{\psi} \in \boldsymbol{V}_{0}$. More precisely

$$
-\int_{\Gamma}\left(\boldsymbol{v}_{\Gamma} \cdot \boldsymbol{n} \psi_{P}+\boldsymbol{v}_{\Gamma} \cdot \boldsymbol{\tau} \psi_{S}\right) \mathrm{d} \sigma=m_{\Gamma}\left(\partial_{t}^{2} \boldsymbol{\varphi}, \boldsymbol{\psi}\right),
$$

where we define the bilinear form

$$
m_{\Gamma}(\boldsymbol{\varphi}, \boldsymbol{\psi}):=\frac{1}{2 V_{S}^{2}} \int_{\Gamma}(\mathcal{I}(\boldsymbol{\varphi} \cdot \boldsymbol{n}) \boldsymbol{\psi} \cdot \boldsymbol{\tau}-\mathcal{I}(\boldsymbol{\varphi} \cdot \boldsymbol{\tau}) \boldsymbol{\psi} \cdot \boldsymbol{n}) \mathrm{d} \boldsymbol{\gamma}
$$

Finally substituting 75 into $(73)$, we see that $\varphi$ is solution of the following variational problem

$$
\left\{\begin{array}{l}
\text { Find } \boldsymbol{\varphi}(t): \mathbb{R}^{+} \longrightarrow \boldsymbol{V}_{0}, \text { satisfying }(71) \text { and such that } \\
\frac{\mathrm{d}^{2}}{\mathrm{~d} t^{2}} m(\boldsymbol{\varphi}(t), \boldsymbol{\psi})+a(\boldsymbol{\varphi}(t), \boldsymbol{\psi})=l(t, \boldsymbol{\psi}), \quad \forall \boldsymbol{\psi} \in \boldsymbol{V}_{0},
\end{array}\right.
$$

where the new mass bilinear form $m(\cdot, \cdot)$ is defined by

$$
m(\boldsymbol{\varphi}, \boldsymbol{\psi})=m_{\Omega}(\boldsymbol{\varphi}, \boldsymbol{\psi})+m_{\Gamma}(\boldsymbol{\varphi}, \boldsymbol{\psi}) .
$$

\subsubsection{Well-posedness issues}

At a first glance, the variational problem (77) looks like a nice hyperbolic variational problem in the sense of theory of Lions-Magenes [11. We already saw that the bilinear form $a(\cdot, \cdot)$ is continuous and coercive in $\boldsymbol{V}$. Another good point is that the bilinear form $m_{\Gamma}(\cdot, \cdot)$ is symmetric (so $m(\cdot, \cdot)$ is too) due to the observation that, using integration by parts along $\Gamma$, we can write, for all $(\boldsymbol{\varphi}, \boldsymbol{\psi}) \in \boldsymbol{V}_{0} \times \boldsymbol{V}_{0}$

$$
m_{\Gamma}(\boldsymbol{\varphi}, \boldsymbol{\psi})=\frac{1}{2 V_{S}^{2}} \int_{\Gamma}(\mathcal{I}(\boldsymbol{\varphi} \cdot \boldsymbol{n}) \boldsymbol{\psi} \cdot \boldsymbol{\tau}+\mathcal{I}(\boldsymbol{\psi} \cdot \boldsymbol{n}) \boldsymbol{\varphi} \cdot \boldsymbol{\tau}) \mathrm{d} \boldsymbol{\gamma}
$$

In addition one observes that $m_{\Gamma}(\cdot, \cdot)$ is continuous in $\boldsymbol{V}_{0}$ because the operator $\mathcal{I}$ maps continuously $H^{-1 / 2}(\Gamma)$ into $H^{1 / 2}(\Gamma)$. Another nice property of $m(\cdot, \cdot)$ is given by the following lemma. 
Lemma 3.5. We have the injectivity result

$$
\text { (i) } m(\boldsymbol{\varphi}, \boldsymbol{\psi})=0 \quad \forall \boldsymbol{\psi} \in \boldsymbol{V}_{0} \quad \Longrightarrow \quad \text { (ii) } \quad \boldsymbol{\varphi}=0 .
$$

Proof. Let $\boldsymbol{\varphi}$ satisfying $(i)$, since $\mathcal{D}(\Omega)^{2} \subset \boldsymbol{V}_{0}$, in particular

$$
\forall \boldsymbol{\psi} \in \mathcal{D}(\Omega)^{2}, \quad m(\boldsymbol{\varphi}, \boldsymbol{\psi})=m_{\Omega}(\boldsymbol{\varphi}, \boldsymbol{\psi})=0
$$

thus $\varphi=0$ by density of $\mathcal{D}(\Omega)^{2}$ in $L^{2}(\Omega)^{2}$.

However, all these properties are not sufficient to fit Lions-Magenes theory which also requires the positivity of $m(\cdot, \cdot)$. Unfortunately, this fails to be true:

Theorem 3.6. Assume that there is a part of the boundary $\Gamma$ that is of class $C^{2}$. Then, therere exists $\boldsymbol{\psi} \in \boldsymbol{V}_{0}$ such that

$$
m(\boldsymbol{\psi}, \boldsymbol{\psi})<0 .
$$

Proof. Assume that the function $\boldsymbol{x}(s)$ that parametrizes $\Gamma$ (see the begining of section 3p satisfies

$$
\boldsymbol{x}(s) \in C^{2}(a, b) \text {, for some }[a, b] \subset[0, L] .
$$

Let $\boldsymbol{n}(s)$ be the unit normal vector to $\Gamma$ at point $\boldsymbol{x}(s)$, outgoing with respect to $\Omega$, and $c(s)$ be the curvature of $\Gamma$ at this point. Let us define $\nu^{+}$such that

$$
\nu^{+}=\frac{1}{2} \sup _{s \in(a, b)} \frac{1}{|c(s)|} .
$$

By elementary differential geometry it is well known that the map

$$
(s, \nu) \in(a, b) \times\left(0, \nu^{+}\right) \rightarrow \boldsymbol{x}(s)-\nu \boldsymbol{n}(s) \in \mathbb{R}^{2}
$$

is injective. Moreover, there exists $0<\nu^{*} \leq \nu^{+}$such that

$$
\Omega_{a, b}^{*}:=\left\{\boldsymbol{x}(s)-\nu \boldsymbol{n}(s), s \in(a, b), \nu \in\left(0, \nu^{*}\right)\right\} \subset \Omega
$$

and that $(s, \nu) \rightarrow \boldsymbol{x}(s)+\nu \boldsymbol{n}(s)$ defines a change of variable from $(a, b) \times\left(0, \nu^{*}\right)$ into $\Omega_{a, b}^{*}$ with jacobian $J(s, \nu):=1-\nu c(s)$ that is uniformly bounded by $J^{*}$ on $(a, b) \times\left(0, \nu^{*}\right)$. Let $\theta \in \mathcal{D}(a, b)$ and $\theta \neq 0$ such that

$$
\int_{a}^{b} \theta(s) d s=0
$$

and $\chi \in C^{\infty}\left(\mathbb{R}^{+}\right)$such that supp $\chi \subset[0,1]$ and $\chi(0)=1$. Let $0<\delta<\nu^{*}$ be a small parameter devoted to tend to 0 , we define $\psi_{\delta} \in C^{1}(\Omega)^{2}$ as

$$
\boldsymbol{\psi}_{\delta}(\boldsymbol{x})= \begin{cases}\left(\theta(s) \boldsymbol{\tau}(s)-\theta^{\prime}(s) \boldsymbol{n}(s)\right) \chi(\nu / \delta), & \text { if } \boldsymbol{x}=\boldsymbol{x}(s)-\nu \boldsymbol{n}(s) \in \Omega_{a, b}^{*}, \\ 0, & \text { else }\end{cases}
$$


Thanks to the assumption on $\theta, \boldsymbol{\psi}_{\delta}$ belongs to $\boldsymbol{V}_{0}$. On the one hand

$$
m_{\Omega}\left(\boldsymbol{\psi}_{\delta}, \boldsymbol{\psi}_{\delta}\right) \leq \delta\left(J^{*} / V_{S}^{2}\right)\|\chi\|_{L^{2}\left(\mathbb{R}^{+}\right)}^{2}\|\theta\|_{H^{1}(a, b)}^{2} .
$$

On the other hand, along $\Gamma, \boldsymbol{\psi}_{\delta} \cdot \boldsymbol{\tau}=\theta(s)$ for $s \in(a, b)$ and 0 otherwise. In the same way $\boldsymbol{\psi}_{\delta} \cdot \boldsymbol{n}=-\theta^{\prime}(s)$ for $s \in(a, b)$ and 0 otherwise, so that $\mathcal{I}\left(\boldsymbol{\psi}_{\delta} \cdot \boldsymbol{n}\right)=$ $-\theta(s)$ for $s \in(a, b)$ and 0 otherwise. Therefore

$$
m_{\Gamma}\left(\boldsymbol{\psi}_{\delta}, \boldsymbol{\psi}_{\delta}\right)=-\|\theta\|_{L^{2}(a, b)}^{2} / V_{S}^{2} .
$$

Comparing (82) and 83 it is clear that $m\left(\boldsymbol{\psi}_{\delta}, \boldsymbol{\psi}_{\delta}\right)$ is strictly negative for $\delta$ small enough.

Remark 3.7. The technical assumption on Theorem 3.6 about the local regularity of $\Gamma$ is most likely unnecessary (and at the same time not very restrictive in practice) but needed for the proof above.
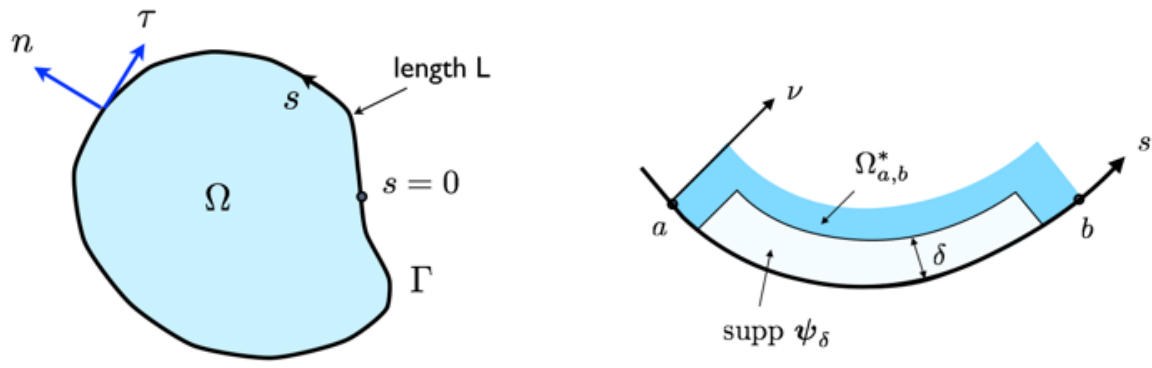

Figure 1: Left: Definition of the parametrization of the boundary. Right: Definition of the curvilinear coordinates and notations for the construction of the function $\psi_{\delta}$ in the proof of Theorem 3.6 .

As one can expect, the property of Theorem 3.6 is the cause of severe instabilities for any finite element approximation of the variational formulation (77). This will be put in evidence in the next section.

\subsubsection{Numerical instabilities of Galerkin discretizations}

Introduction. We introduce a finite dimensional approximation of the space $\boldsymbol{V}_{0}$ that will be denoted by $\boldsymbol{V}_{0 h}$ that is constructed as follows

$$
\boldsymbol{V}_{0 h}=\boldsymbol{V}_{h} \cap \boldsymbol{V}_{0}
$$

where $\boldsymbol{V}_{h}=V_{P, h} \times V_{S, h}$ is a Galerkin approximation of the space $H^{1}(\Omega)^{2}$. The problem to solve is

$$
\left\{\begin{array}{l}
\text { Find } \boldsymbol{\varphi}_{h}(t): \mathbb{R}^{+} \longrightarrow \boldsymbol{V}_{0 h} \text { such that }\left(\boldsymbol{\varphi}_{h}, \partial_{t} \boldsymbol{\varphi}_{h}\right)(t=0)=(\mathbf{0}, \mathbf{0}) \text { and } \\
\frac{\mathrm{d}^{2}}{\mathrm{~d} t^{2}} m_{h}\left(\boldsymbol{\varphi}_{h}(t), \boldsymbol{\psi}_{h}\right)+a\left(\boldsymbol{\varphi}_{h}(t), \boldsymbol{\psi}_{h}\right)=l\left(t, \boldsymbol{\psi}_{h}\right), \quad \forall \boldsymbol{\psi}_{h} \in \boldsymbol{V}_{0 h},
\end{array}\right.
$$


where $m_{h}(\cdot, \cdot)$ is an approximation of the bilinear form $m(\cdot, \cdot)$ (for instance it can be computed using quadrature formulae). At the algebraic level the formulation takes the following form

$$
\mathbb{M}_{h} \frac{\mathrm{d}^{2} \boldsymbol{\Phi}_{h}}{\mathrm{~d} t^{2}}+\mathbb{A}_{h} \boldsymbol{\Phi}_{h}=\boldsymbol{F}_{h}, \quad \mathbb{M}_{h}:=\mathbb{M}_{h}^{\Omega}+\mathbb{M}_{h}^{\Gamma}, \quad \mathbb{A}_{h}:=\mathbb{A}_{h}^{\Omega}+\mathbb{A}_{h}^{\Gamma}
$$

where as in 43$), \boldsymbol{\Phi}_{h}^{t}=\left(\boldsymbol{\Phi}_{P, h}, \boldsymbol{\Phi}_{S, h}\right)^{t}$ is the vectors of degrees of freedom of $\varphi_{P, h}$ and $\varphi_{S, h}$. If one thinks of continuous Lagrange finite elements for instance, the matrix $\mathbb{M}_{h}^{\Gamma}$ has the following structure

$$
\mathbb{M}_{h}^{\Gamma}=\left(\begin{array}{cc}
\mathbb{M}_{h}^{\Gamma, P} & \mathbb{M}_{h}^{\Gamma, P S} \\
\left(\mathbb{M}_{h}^{\Gamma, P S}\right)^{t} & \mathbb{M}_{h}^{\Gamma, S}
\end{array}\right)
$$

where the only non-zero values entries of the matrices $\left(\mathbb{M}_{h}^{\Gamma, P}, \mathbb{M}_{h}^{\Gamma, S}, \mathbb{M}_{h}^{\Gamma, P S}\right)$ correspond to the degrees of freedom located on the boundary $\Gamma$ (note that due to the double integral on the boundary, each degree of freedom on the boundary is coupled with all the other degrees of freedom on the boundary).

Despite of the injectivity property 80 , it is not clear that the matrix $\mathbb{M}_{h}$ is invertible (which is a necessary property if one wants to use an explicit scheme in time). Indeed the proof was done at the continuous level using density properties of smooth compactly supported functions. At the discrete level, i.e. in finite dimensional space, such an argument can not be used any more.

Moreover, even in the case where $\mathbb{M}_{h}$ is invertible, it is most likely that, because of the result of Theorem 3.6. the solution of the semi-discrete evolution problem, which is given by

$$
\boldsymbol{\Phi}_{h}(t)=\int_{0}^{t}\left(\mathbb{M}_{h}^{-1} \mathbb{A}_{h}\right)^{-\frac{1}{2}} \sin \left(\left(\mathbb{M}_{h}^{-1} \mathbb{A}_{h}\right)^{\frac{1}{2}}(t-s)\right) \mathbb{M}_{h}^{-1} \boldsymbol{F}_{h}(s) d s,
$$

will blow up exponentially in time (i.e. the semi-discrete scheme (86) is unstable). Such a phenomenen is linked to the existence of strictly negative eigenvalues for the following symmetric eigenvalue problem (note that, as soon as $\mathbb{M}_{h}$ is invertible, such eigenvalues are necessarily real)

$$
\text { Find } \boldsymbol{\Psi}_{h} \neq 0 \text { and } \lambda \in \mathbb{R} \text { such that } \mathbb{A}_{h} \boldsymbol{\Psi}_{h}=\lambda \mathbb{M}_{h} \boldsymbol{\Psi}_{h}
$$

Of course, the rate of exponential blow up will be, at least, given by the most negative eigenvalue $\lambda$ of $\mathbb{M}_{h}^{-1} \mathbb{A}_{h}$. More precisely, if $\boldsymbol{\sigma}_{h}$ denotes the spectrum of $\mathbb{M}_{h}^{-1} \mathbb{A}_{h}$ with minimum value $\lambda_{-}(h)<0$, then

$$
\left\|\left(\mathbb{M}_{h}^{-1} \mathbb{A}_{h}\right)^{-\frac{1}{2}} \sin \left(\left(\mathbb{M}_{h}^{-1} \mathbb{A}_{h}\right)^{\frac{1}{2}} t\right)\right\| \geq C e^{t \sqrt{|\mathcal{C}(h)|}}
$$

where $\mathcal{C}(h) \in \mathbb{R}$ is such that $0<\mathcal{C}(h)<\left|\lambda_{-}(h)\right|$. In the following, we come back in more details on these invertibility and stability issues. In particular, in 
the next paragraph we consider a simplified toy problem associated to a simple geometry. In this case, the two issues (invertibility and lack of positivity) can be studied analytically. Then, in the last paragraph of this section we consider a more general finite element discretization and illustrate the instability of the scheme through numerical computations.

Remark 3.8. Note that because of the gauge condition (70) (vii) the two spaces are coupled at the boundary. In practice the gauge condition is imposed using a Lagrange multiplier as usually done when imposing zero average condition [19].

Study of a particular toy problem. We want to solve elastodynamic equations (2) on the cylinder

$$
\Omega=(0,+\infty) \times(-\pi, \pi)
$$

obtained when identifying the upper and lower boundaries, where $x_{1}=0$ is the free boundary. In consequence, we impose periodic boundary conditions

$$
\boldsymbol{u}\left(t, x_{1}, \pi\right)=\boldsymbol{u}\left(t, x_{1},-\pi\right), \partial_{2} \boldsymbol{u}\left(t, x_{1}, \pi\right)=\partial_{2} \boldsymbol{u}\left(t, x_{1},-\pi\right), \quad \forall x_{1} \in \mathbb{R}^{+},
$$

in such a way that the boundary of $\Omega$ can be identified to a circle (this is a closed curve). Note that this particular example does not completely fit the assumptions made on the domain's geometry since $\Omega$ is unbounded, however the reader will easily convince himself that this is not an essential issue. Next we discretize the space $H^{1}(\Omega)$ as follows. We denote $V_{h} \subset H^{1}\left(\mathbb{R}^{+}\right)$the uniform discretization of $\mathbb{R}^{+}$by $\mathbb{P}_{1}$ finite elements of length $h$, i.e.

$$
V_{h}:=\left\{\psi_{h} \in H^{1}\left(\mathbb{R}^{+}, \mathbb{C}\right) \text { s.t. } \psi_{h}\left(x_{1}\right)=\sum_{j=0}^{+\infty} \psi^{j} w_{j}\left(x_{1}\right)\right\}
$$

where the set $\left\{w_{j}\right\}$ are the piecewise affine functions that satisfy $w_{j}(k h)=\delta_{k j}$ (where $\delta$ is the Kronecker symbol) so that $\psi^{j}=\psi_{h}(j h)$. Note that this space is isomorphic to the space $\ell^{2}(\mathbb{N})$. In the direction $x_{2}$, we use a spectral method consisting in truncating the natural Fouirier series expansion of a function in $L^{2}(-\pi, \pi)$ at order $L>0$, where $2 \pi / L$ is thus the minimal oscillation length allowed in the approximate space. This corresponds to the following Galerkin approximation space $\boldsymbol{V}_{h}=V_{h}^{L} \times V_{h}^{L}$ with

$$
V_{h}^{L}:=\left\{\varphi_{h} \in H^{1}(\Omega, \mathbb{R}) \text { s.t. } \varphi_{h}=\sum_{\ell=-L}^{L} \varphi_{h}^{\ell}\left(x_{1}\right) e^{-i \ell x_{2}} \text { with } \varphi_{h}^{\ell} \in V_{h}\right\} .
$$

Note that the approximation parameter is the couple $(h, L)$ where $h$ is the space step in $x_{1}$, devoted to tend to 0 , and $L$ the truncation parameter in frequency, devoted to tend to $+\infty$ (see also remark 3.9 ). It is then immediate to see that the space $\boldsymbol{V}_{0 h}$ (see (84)) is nothing but

$$
\boldsymbol{V}_{0 h}=\left\{\boldsymbol{\varphi}_{h}=\left(\varphi_{P, h}, \varphi_{S, h}\right) \in \boldsymbol{V}_{h} \text { s.t. } \varphi_{P}^{0,0}=\varphi_{S}^{0,0}=0\right\} \subset \boldsymbol{V}_{0} .
$$


where $\varphi_{P}^{0,0}:=\varphi_{P, h}^{0}(0)$ and $\varphi_{S}^{0,0}:=\varphi_{S, h}^{0}(0)$, according to the notation introduced above.

Remark 3.9. If one makes an analogy with $\mathbb{Q}_{1}$ finite elements for instance, this would corresponds to discretise $\Omega$ uniformly with rectangular finite elements of length $h_{1}=h$ in the direction $x_{1}$ and $h_{2}=\pi / 2 L$ in the direction $x_{2}$.

Then we look for the solution of 85 with the following expression of the bilinear form $a(\cdot, \cdot)$ and $m(\cdot, \cdot)$ that take into account the fact that we deal with complex valued functions,

$$
\left\{\begin{aligned}
m_{h}\left(\boldsymbol{\varphi}_{h}, \boldsymbol{\psi}_{h}\right)= & \frac{1}{V_{P}^{2}} \int_{-\pi}^{\pi} \oint_{\mathbb{R}^{+}} \varphi_{P, h} \overline{\psi_{P, h}} d x_{1} d x_{2}+\frac{1}{V_{S}^{2}} \int_{-\pi}^{\pi} \oint_{\mathbb{R}^{+}} \varphi_{S, h} \overline{\psi_{S, h}} d x_{1} d x_{2} \\
& -\frac{1}{2 V_{S}^{2}} \int_{-\pi}^{\pi}\left[\left(\int_{-\pi}^{x_{2}} \varphi_{P, h} d s\right) \overline{\psi_{S, h}}+\left(\int_{-\pi}^{x_{2}} \overline{\psi_{P, h}} d s\right) \varphi_{S, h}\right] \mathrm{d} x_{2}, \\
a\left(\boldsymbol{\varphi}_{h}, \boldsymbol{\psi}_{h}\right)= & \int_{\Omega}\left(\nabla \varphi_{P, h}+\operatorname{curl} \varphi_{S, h}\right) \cdot\left(\nabla \overline{\psi_{P, h}}+\operatorname{curl} \overline{\psi_{S, h}}\right) \mathrm{d} \boldsymbol{x},
\end{aligned}\right.
$$

where the symbol $\oint$ is used to account for the use of the following quadrature formula

$$
\oint_{\mathbb{R}^{+}} \varphi_{h} \overline{\psi_{h}} d x_{1}:=\frac{h}{2} \varphi^{0} \overline{\psi^{0}}+h \sum_{j=1}^{+\infty} \varphi^{j} \overline{\psi^{j}}, \quad\left\|\varphi_{h}\right\|_{h}^{2}:=\oint_{\mathbb{R}^{+}}\left|\varphi_{h}\right|^{2} d x_{1},
$$

that allows us to get mass lumping. Since we have used a spectral approximation in $x_{2}$, the previous problem decouples as a family of $2 L+1$ problems in $1 D$ with respective unknowns $\left\{\boldsymbol{\varphi}_{h}^{\ell}:=\left(\varphi_{P, h}^{\ell}, \varphi_{S, h}^{\ell}\right)\right\}$. More precisely, choosing

$$
\boldsymbol{\psi}_{h}=e^{-i \ell x_{2}} \boldsymbol{\psi}_{h}^{\ell}\left(x_{1}\right) \quad \text { with } \quad \boldsymbol{\psi}_{h}^{\ell}:=\left(\psi_{P, h}^{\ell}, \psi_{S, h}^{\ell}\right)
$$

as test functions in (85) and using the orthogonality properties of trigonometric functions, we get that, for each $\ell \in\{-L, \ldots, L\}, \varphi_{h}^{\ell}(t): \mathbb{R}^{+} \mapsto V_{h} \times V_{h}$ satisfies the following 1D variational problem: for all $\boldsymbol{\psi}_{h}^{\ell} \in V_{h} \times V_{h}$,

$$
\frac{d^{2}}{d t^{2}} m_{\ell}\left(\boldsymbol{\varphi}_{h}^{\ell}, \boldsymbol{\psi}_{h}^{\ell}\right)+a_{\ell}\left(\boldsymbol{\varphi}_{h}^{\ell}, \boldsymbol{\psi}_{h}^{\ell}\right)=l_{\ell}\left(t, \boldsymbol{\psi}_{h}^{\ell}\right),
$$


where $m_{\ell}(\cdot, \cdot)=m_{\ell, \Omega}(\cdot, \cdot)+m_{\ell, \Gamma}(\cdot, \cdot)$ and for $l \in\{-L, \ldots, L\}$

$$
\left\{\begin{aligned}
m_{\ell, \Omega}\left(\boldsymbol{\varphi}_{h}^{\ell}, \boldsymbol{\psi}_{h}^{\ell}\right) & =\frac{1}{V_{P}^{2}} \oint_{\mathbb{R}^{+}} \varphi_{P, h}^{\ell} \overline{\psi_{P, h}^{\ell}} d x_{1}+\frac{1}{V_{S}^{2}} \oint_{\mathbb{R}^{+}} \varphi_{S, h}^{\ell} \overline{\psi_{S, h}^{\ell}} d x_{1}, \\
m_{\ell, \Gamma}\left(\boldsymbol{\varphi}_{h}^{\ell}, \boldsymbol{\psi}_{h}^{\ell}\right)= & \frac{i}{2 V_{S}^{2} \ell} \varphi_{S}^{\ell, 0} \overline{\psi_{P}^{\ell, 0}}-\frac{i}{2 V_{S}^{2} \ell} \varphi_{P}^{\ell, 0} \overline{\psi_{S}^{\ell, 0}}, \quad \forall l \neq 0, \\
a_{\ell}\left(\boldsymbol{\varphi}_{h}^{\ell}, \boldsymbol{\psi}_{h}\right)= & \int_{\mathbb{R}^{+}}\left(\partial_{1} \varphi_{P, h}^{\ell}-i \ell \varphi_{S, h}^{\ell}\right)\left(\partial_{1} \overline{\psi_{P, h}^{\ell}}+i \ell \overline{\psi_{S, h}^{\ell}}\right) d x_{1} \\
& +\int_{\mathbb{R}^{+}}\left(\partial_{1} \varphi_{S, h}^{\ell}+i \ell \varphi_{P, h}^{\ell}\right)\left(\partial_{1} \overline{\psi_{S, h}^{\ell}}-i \ell \overline{\psi_{P, h}^{\ell}}\right) d x_{1}, \\
l_{\ell}\left(t, \boldsymbol{\psi}_{h}\right)= & -\int_{\mathbb{R}^{+}} g_{l}^{1}\left(\partial_{1} \overline{\bar{\psi}_{P, h}^{\ell}}+i \ell \overline{\bar{\psi}_{S, h}^{\ell}}\right) d x_{1} \\
& +\int_{\mathbb{R}^{+}} g_{l}^{2}\left(\partial_{1} \overline{\psi_{S, h}^{\ell}}-i \ell \overline{\psi_{P, h}^{\ell}}\right) d x_{1},
\end{aligned}\right.
$$

with

$$
\boldsymbol{g}=\left(g^{1}, g^{2}\right)^{t}=\left(\sum_{\ell=-L}^{L} g_{\ell}^{1}\left(x_{1}\right) e^{-i \ell x_{2}}, \sum_{\ell=-L}^{L} g_{\ell}^{2}\left(x_{1}\right) e^{-i \ell x_{2}}\right)^{t} .
$$

Note that, in (86), $\mathbb{M}_{h}$ and $\mathbb{A}_{h}$ are no longer matrices but operators that have an orthogonal decomposition of the form

$$
\mathbb{M}_{h}=\bigoplus_{\ell=-L}^{L} \mathbb{M}_{h, \ell}, \quad \mathbb{A}_{h}=\bigoplus_{\ell=-L}^{L} \mathbb{A}_{h, \ell}
$$

where each $\mathbb{M}_{h, \ell}$ or $\mathbb{A}_{h, \ell} \in \mathcal{L}\left(V_{h} \times V_{h}\right)$ is represented by an infinite matrix.

First, we study the invertiblity of $\mathbb{M}_{h}$. We have the following results:

- For some choice of the approximation parameters $(h, L)$ (see 100 below), the operator $\mathbb{M}_{h}$ may be not invertible.

- Under the following condition on the parameters $(h, L)$

$$
h^{2} L^{2}<V_{P}^{2} / V_{S}^{2},
$$

the operator $\mathbb{M}_{h}$ is invertible. Note that the condition (97) can be interpreted as a non degeneracy condition about the computational mesh. Indeed, according to the analogy of remark 3.9, the condition (97) means $h_{1} / h_{2}$ small enough, i.e. that the rectangle $\left[0, h_{1}\right] \times\left[0, h_{2}\right]$ is not too flattened in the $x_{2}$ direction.

Remark 3.10. Since $V_{P}^{2} \geq 2 V_{S}^{2}$ the condition $h<\frac{\sqrt{2}}{L}$ always ensures and is thus a sufficient conditon for the ivertibility of $M_{h}$ for any elastic material. 
In order to prove the assertions above, we note that, from $(96), \mathbb{M}_{h}$ is not invertible if and only if one of the $\mathbb{M}_{h, \ell}$ is not invertible, or equivalently if, for some $-L \leq \ell \leq L$ there exists $\varphi_{h}^{\ell} \neq 0$ such that

$$
m_{\ell}\left(\boldsymbol{\varphi}_{h}^{\ell}, \boldsymbol{\psi}_{h}\right)=0, \quad \forall \boldsymbol{\psi}_{h} \in V_{h} \times V_{h}
$$

Let $\varphi_{h}^{\ell}$ satisfying 98 . If $\ell=0$, thanks to the use of the quadrature rule $(93)$, the infinite matrix $\mathbb{M}_{h, 0}$ is purely diagonal with non zero diagonal entries. Therefore $(98)$ implies $\varphi_{h}^{0}=0$. For $\ell \neq 0,98$ is equivalent to

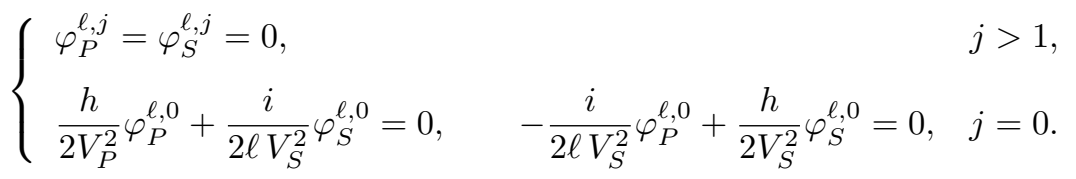

As a consequence, the invertibility of $\mathbb{M}_{h, \ell}$ is equivalent to the invertibility of the $2 \times 2$ system of the second line of $(99)$. From the inspection of this system, we see that

$$
\mathbb{M}_{h} \text { is not invertible } \Leftrightarrow \exists 1 \leq \ell \leq L \text { such that } \ell^{2} h^{2}=V_{P}^{2} / V_{S}^{2},
$$

and that the condition (97) thus ensures the invertibility of $\mathbb{M}_{h}$.

Let us now investigate the lack of positivity of the bilinear form $m_{h}(\cdot, \cdot)$, which is clearly directly related to the instability of the differential equation 86 . We will prove the result for each $\mathbb{M}_{h, l}$. We proceed in a constructive way as in the proof of Theorem 3.6 by considering a discrete field

$$
\phi_{h}^{\ell}:=\left(\phi_{P, h}^{\ell}, \phi_{S, h}^{\ell}\right) \in V_{h} \times V_{h}
$$

which is as concentrated as possible to $x_{1}=0$, which correponds to $\left(w_{0}(\cdot)\right.$ being the $\mathbb{P}_{1}$ Lagrange basis function associated to the first node of the discretization in $x_{1}$ )

$$
\phi_{P, h}^{\ell}\left(x_{1}\right):=w_{0}\left(x_{1}\right), \quad \phi_{S, h}^{\ell}\left(x_{1}\right):=i w_{0}\left(x_{1}\right),
$$

where the factor $i$ for $\phi_{S, h}^{\ell}\left(x_{1}\right)$ is chosen on purpose to give $m_{\ell, \Gamma}\left(\phi_{h}^{\ell}, \phi_{h}^{\ell}\right)<0$. Indeed, according to 95 ,

$$
m_{\ell, \Gamma}\left(\phi_{h}, \phi_{h}\right)=-1 /\left(\ell V_{S}^{2}\right)
$$

and adding $m_{\ell, \Omega}\left(\phi_{h}, \phi_{h}\right)$, one easily obtains, setting $h_{*}:=\frac{2 V_{P}^{2}}{V_{P}^{2}+V_{S}^{2}} \in\left[\frac{4}{3}, 2\right]$,

$$
m_{\ell}\left(\phi_{h}, \phi_{h}\right)=\frac{h}{2 V_{P}^{2}}+\frac{h}{2 V_{S}^{2}}-\frac{1}{\ell V_{S}^{2}}=\frac{h}{2}\left(\frac{1}{V_{P}^{2}}+\frac{1}{V_{S}^{2}}\right)\left(1-\frac{h_{*}}{\ell h}\right) .
$$

Then, as soon as $h$ is small enough, namely $h<h^{*}$, the set of indices $\ell$ for which $m_{\ell}\left(\phi_{h}, \phi_{h}\right)<0$ is non empty, which shows in particular that $\mathbb{M}_{h}$ is no longer positive. More precisely

$$
\left\{1 \leq \ell \leq L / m_{\ell}\left(\phi_{h}, \phi_{h}\right)<0\right\}=\{1, \cdots, L(h)\},
$$


where

$$
L(h):=\min \left\{L,\left\lceil h_{*} / h\right\rceil-1\right\} .
$$

Then, assuming that $h<h_{*}$, we can investigate the exponential blow up of the solution of (86). Let us first notice that, if $\boldsymbol{\sigma}_{\ell, h}$ denotes the spectrum of the operator $\mathbb{M}_{h, \ell}^{-1} \mathbb{A}_{h, \ell}$, we deduce from (104) that

$$
\forall 1 \leq \ell \leq L(h), \quad \sigma_{\ell, h} \cap(-\infty, 0) \neq \emptyset .
$$

In view of formula (95), it is clear that the quadratic form $m_{\ell, \Gamma}\left(\varphi_{h}, \varphi_{h}\right)$ cannot remain negative on a two dimensional subspace of $V_{h} \times V_{h}$. As a consequence, for $1 \leq \ell \leq L(h), \sigma_{\ell, h} \cap(-\infty, 0)$ is made of one single eigenvalue denoted $\lambda_{\ell}^{-}(h)$ As a consequence, the lowerbound 88 holds true with

$$
\lambda_{-}(h)=\min _{1 \leq \ell \leq L(h)} \lambda_{\ell}^{-}(h) .
$$

For classical self-adjoint problem it is well known that eigenvalues are related to Rayleigh quotients and it is tempting to compute the Rayleigh quotients of each of the test field $\phi_{h}^{\ell}$ defined by 101,102 .

One computes that $a_{\ell}\left(\phi_{h}^{\ell}, \phi_{h}^{\ell}\right)=\frac{2}{h}\left(1-\ell h+\frac{\ell^{2} h^{2}}{3}\right)$, so that

$$
\frac{a_{\ell}\left(\phi_{h}^{\ell}, \phi_{h}^{\ell}\right)}{m_{\ell}\left(\phi_{h}^{\ell}, \phi_{h}^{\ell}\right)}=\frac{4}{h^{2}}\left(1-\ell h+\frac{\ell^{2} h^{2}}{3}\right)\left(\frac{1}{V_{P}^{2}}+\frac{1}{V_{S}^{2}}\right)^{-1}\left(1-\frac{h_{*}}{\ell h}\right)^{-1} .
$$

We see that for constant $\ell h$ the above Rayleigh quotient blows up (in absolute value) as $C / h^{2}$ which makes us conjecture that $\lambda_{-}(h)$ is negative and decreases when $h$ decreases .

Remark 3.11. The fact that the quadratic form $m(\cdot, \cdot)$ has no sign prevent us from using the well-known standard min-max principle for classical self-adjoint spectral problems.

Let us illustrate the blow-up phenomenon via numerical simulations. For these simulations we used the following parameters

$$
\lambda=20, \quad \mu=4, \quad \rho=1, \quad L=60, \quad h=\frac{\pi}{200}
$$

and a source located close to the boundary. We have taken the leap frog scheme for the time discretization, which ensures a finite propagation velocity in the direction $x_{1}$ for the numerical solution. Of course, we have chosen a bounded domain in the direction $x_{1}$ but truncated this domain at $x_{1}=x_{1, \max }$ in such a way that the numerical solution does not reach the boundary $x_{1}=x_{1, \max }$ before the final time $T$ of the computation. In Figure 2, we plot snapshots of the solution obtained at three different simulation times. These results illustrate 
the bad behavior of the semi-discrete problem since one can observe blow-up of the solution that is initiated, as expected, close to the boundary but propagates inside the computational domain as $t$ increases (the color scale is saturated at the boundary).
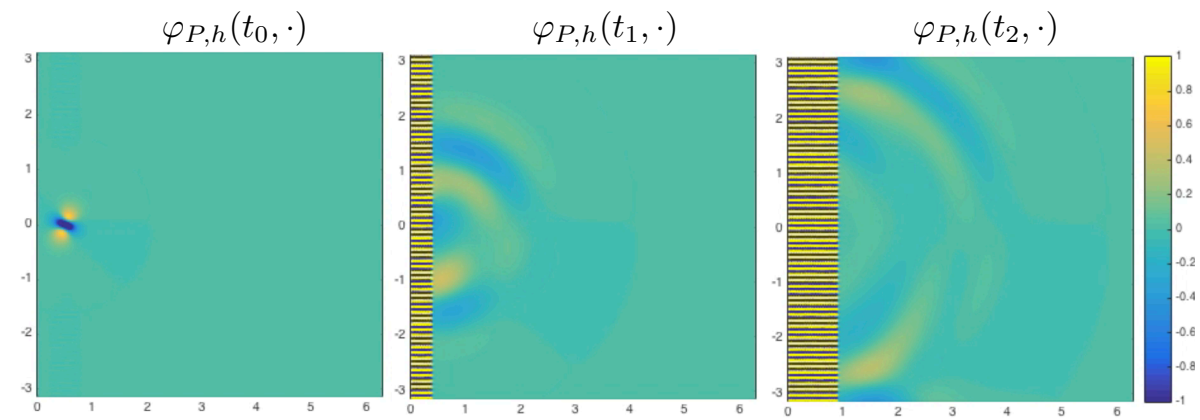

Figure 2: Snapshots at three different times $t_{0}<t_{1}<t_{2}$ of the solution of (85) using a spectral approximation in $x_{2}$ and $\mathbb{P}_{1}$ finite elements in $x_{1}$.

The case of $\mathbb{P}_{1}$ finite element discretizations. We now consider the case of full $\mathbb{P}_{1}$ finite element discretization on triangular meshes of stepsize $h$ of a polygonal domain $\Omega$ and investigate the same questions as in the previous paragraph by means of numerical experiments, allowing us to conjecture that the observations made on the toy problem are still valid in more general situations.

More precisely, we investigate the case where $\Omega$ is the square $[0,1] \times[0,1]$ and the physical parameters are $\lambda=20, \mu=15, \rho=1$. Five different quasiregular meshes have been considered, corresponding approximately to (see also the pictures in Figure 3 and Figure 4

Mesh $1: h \simeq 1 / 3, \quad$ Mesh $2: h \simeq 1 / 7, \quad$ Mesh $3: h \simeq 1 / 15$,

$$
\text { Mesh } 4: h \simeq 1 / 30, \quad \text { Mesh } 5: h \simeq 1 / 60 .
$$

For all meshes, the invertibility of the matrix $\mathbb{M}_{h}$ has been observed experimentally. On the other hand, to check the instability of the semi-discrete problem, we solved numerically (using MATLAB) the eigenvalue problem (87), i.e. computed the spectrum $\boldsymbol{\sigma}_{h}$ of the matrix, which allowed us to check that

- $\lambda_{-}(h)$ is negative and decreases when $h$ decreases (see Figure 5 ) as it was conjectured to be the case for the toy problem. This results into imaginary values for the set $\left\{ \pm \sqrt{\lambda}, \lambda \in \sigma_{h}\right\}$ as illustrated for Meshes 1 and 2 in Figure 3 This set contains more and more imaginary values as soon as $h$ decreases.

- The eigenmode corresponding to the smallest eigenvalue $\lambda_{-}(h)$ is concentrated close to the boundary (the finer the mesh, the more concentrated at 
the boundary). At the same time, we also observe that the eigenmode oscillates more and more along the boundary, this phenomena is represented for Meshes 3, 4 and 5 in Figure 4.

To complement these observations, we have computed an approximation of the solution $\boldsymbol{\Phi}_{h}(t)$ of (86) using finite differences in time. To dispel the idea that the observed instability could be due to the time discretization, we have used the following fully implicit scheme

$$
\mathbb{M}_{h} \frac{\boldsymbol{\Phi}_{h}^{n+1}-2 \boldsymbol{\Phi}_{h}^{n}+\boldsymbol{\Phi}_{h}^{n-1}}{\Delta t^{2}}+\mathbb{A}_{h} \frac{\boldsymbol{\Phi}_{h}^{n+1}+2 \boldsymbol{\Phi}_{h}^{n}+\boldsymbol{\Phi}_{h}^{n-1}}{4}=\boldsymbol{F}_{h}^{n},
$$

In each case, we observed the exponential blow-up of the solution that we can quantify by representing the variations of the sum of the energies associated to the scalar wave equations (see (34)), namely: $t^{n} \mapsto E_{P}\left(t^{n}\right)+E_{S}\left(t^{n}\right)$, computed with $\Delta t=0.005$ for the three meshes, in log-log scale (see Figure 5). The source term is a space/time pulse centered close to the boundary. This confirms that the rate of blow-up increases in when $h$ decreases.
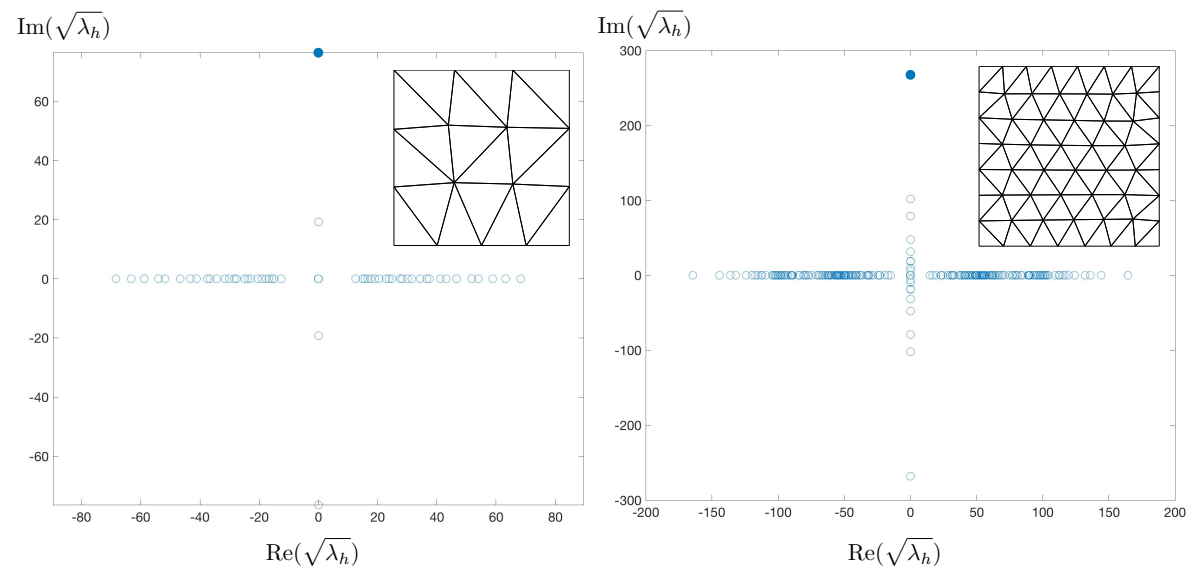

Figure 3: Eigenvalues of (87) in the complex plane for Meshes 1 and 2.

\subsection{The new variational framework}

The diagnosis of the analysis in the previous section is that the space $\boldsymbol{V}_{0}$ is probably too large in the sense that it allows for the appearance of unstable modes after space discretization, this being linked to the non positivity of the bilinear form $m(\cdot, \cdot)$. The idea for circumventing this problem is to identify an adequate subspace $\boldsymbol{V}_{N}$ of $\boldsymbol{V}_{0}$ (where $N$ holds for Neumann and refers to the free boundary condition) in such a way that 


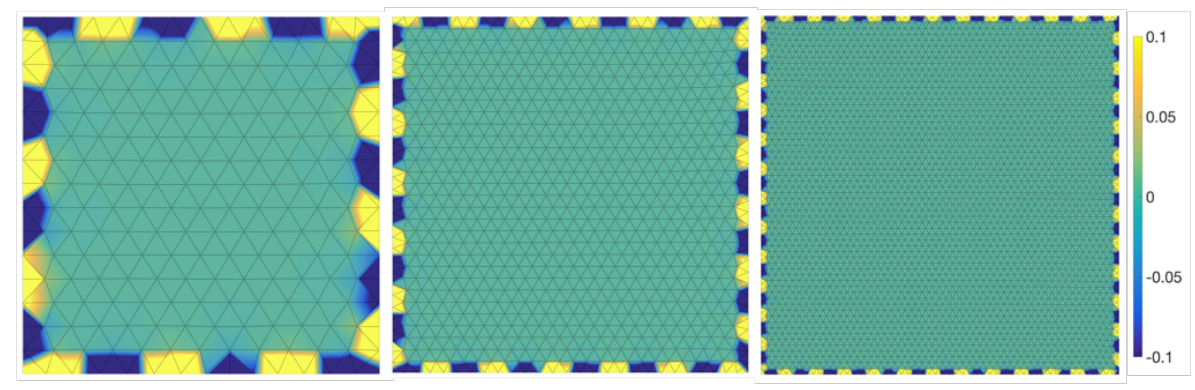

Figure 4: Eigenvector corresponding to the eigenvalue of (87) with largest imaginary part for Meshes 3, 4 and 5 (only the solution corresponding to $\varphi_{P}$ is represented).
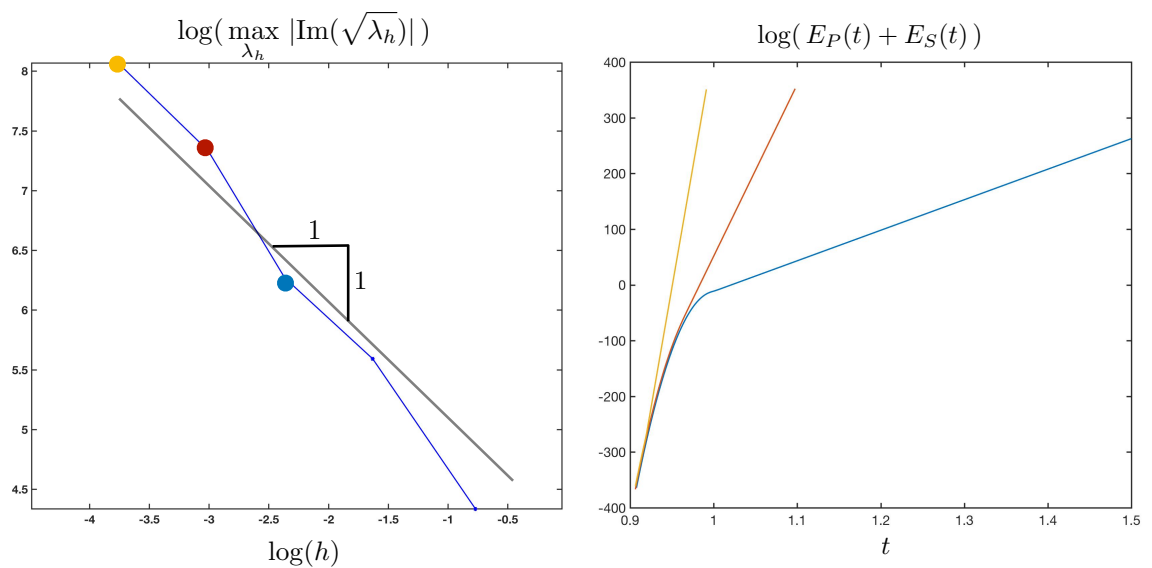

Figure 5: Left: Evolution of the largest imaginary part of the eigenvalues of problem (87) with respect to $h$ (five meshes were considered). Right: Evolution of the norm of the solution with respect to time and for Meshes 3 (in blue), 4 (in red) and 5 (in yellow).

(i) the unknown $\varphi \equiv\left(\varphi_{P}, \varphi_{S}\right)$ defined by (8)-(10) is solution of the variational problem 77 , but set in $\boldsymbol{V}_{N}$, instead of $\boldsymbol{V}_{0}$, i.e.

$$
\left\{\begin{array}{l}
\text { Find } \boldsymbol{\varphi}(t): \mathbb{R}^{+} \longrightarrow \boldsymbol{V}_{N} \text { satisfying }(71) \text { and such that } \\
\frac{\mathrm{d}^{2}}{\mathrm{~d} t^{2}} m(\boldsymbol{\varphi}(t), \boldsymbol{\psi})+a(\boldsymbol{\varphi}(t), \boldsymbol{\psi})=l(t, \boldsymbol{\psi}), \quad \forall \boldsymbol{\psi} \in \boldsymbol{V}_{N} .
\end{array}\right.
$$

(ii) the bilinear form $m(\cdot, \cdot)$ restricted to the space $\boldsymbol{V}_{N}$ is positive definite

$$
\forall \boldsymbol{\psi} \in \boldsymbol{V}_{N} \backslash\{0\}, \quad m(\boldsymbol{\psi}, \boldsymbol{\psi})>0 .
$$


The item (ii) will guide the construction of $\boldsymbol{V}_{N}$ and is expected to guarantee the well-posedness of (108) and the stability of its Galerkin approximation.

\subsubsection{Construction of the new variational space}

The energy naturally associated with (77), which is conserved as soon as the right hand side vanishes, is

$$
\begin{aligned}
E_{N}(t) & :=E_{D}(t)+\frac{1}{2} m_{\Gamma}\left(\partial_{t} \boldsymbol{\varphi}(t), \partial_{t} \boldsymbol{\varphi}(t)\right) \\
& =\frac{1}{2}\left[m\left(\partial_{t} \boldsymbol{\varphi}(t), \partial_{t} \boldsymbol{\varphi}(t)\right)+a(\boldsymbol{\varphi}(t), \boldsymbol{\varphi}(t))\right] .
\end{aligned}
$$

The positivity of this energy is obviously related to the positivity of the bilinear form $m(\cdot, \cdot)$ on a space to which $\varphi$ belongs. For the Dirichlet problem, the (obvious) positivity of the energy $E_{D}(t)$ was confirmed by the identities

$$
\text { (i) } \frac{\rho}{2} m_{\Omega}\left(\partial_{t} \boldsymbol{\varphi}, \partial_{t} \boldsymbol{\varphi}\right)=E_{p}(t) \quad \text { and } \quad(i i) \quad \frac{\rho}{2} a(\boldsymbol{\varphi}, \boldsymbol{\varphi})=E_{c}(t),
$$

where the potential energy $E_{p}(\cdot)$ (resp. kinetic energy $E_{c}(\cdot)$ ) are defined by (37) (resp. (36)). We expect similar identities for the solution of the free boundary problem. In fact, it is clear that the identity (111)(ii) still holds for the solution of the Neumann problem because its proof does not refer to the Dirichlet condition: it only uses (11) which is valid independently of the boundary condition. We just have to obtain an equivalent of 1111)(i) with $m(\cdot, \cdot)$ instead of $m_{\Omega}(\cdot, \cdot)$, i.e.,

$$
\frac{\rho}{2} m\left(\partial_{t} \varphi, \partial_{t} \boldsymbol{\varphi}\right)=E_{p}(t) .
$$

This is related to the identity (38) in Lemma 2.3 which yields

$$
E_{p}(t)=\frac{\rho}{2} m_{\Omega}\left(\partial_{t} \boldsymbol{\varphi}, \partial_{t} \boldsymbol{\varphi}\right)-2 \mu \int_{\Gamma} u_{2} \partial_{\tau} u_{1} \mathrm{~d} \boldsymbol{\gamma},
$$

so that we have to check that, since $2 \mu=2 V_{S}^{2} \rho$,

$$
m_{\Gamma}\left(\partial_{t} \boldsymbol{\varphi}, \partial_{t} \boldsymbol{\varphi}\right)=-4 V_{S}^{2} \int_{\Gamma} u_{2} \partial_{\tau} u_{1} \mathrm{~d} \boldsymbol{\gamma}
$$

Here is where we are going to use the fact that $\boldsymbol{u}$ satisfies the free boundary condition. More precisely, integrating in time (67), we have

$$
\partial_{\tau} u_{1}=\frac{1}{2 V_{S}^{2}} \partial_{t} \boldsymbol{\varphi} \cdot \boldsymbol{\tau}, \quad u_{2}+\frac{1}{2 V_{S}^{2}} \mathcal{I}\left(\partial_{t} \boldsymbol{\varphi} \cdot \boldsymbol{n}\right) \in \mathbb{P}_{0}(\Gamma)
$$

Then, since $\boldsymbol{\varphi}(t)$ belongs to $\boldsymbol{V}_{0},(114)$ follows. The reader should notice that which was important for obtaining (112) is that we could write (see 88 )

$$
\partial_{t} \varphi_{P}=V_{P}^{2} \operatorname{div} \boldsymbol{u}, \quad \partial_{t} \varphi_{S}=-V_{S}^{2} \operatorname{curl} \boldsymbol{u}
$$


with $\boldsymbol{u}$ a smooth enough function satisfying the free boundary condition (51). The above observation gives the idea of the construction of the space $\boldsymbol{V}_{N}$. Let us first introduce the space

$$
\begin{aligned}
\boldsymbol{D} & :=\left\{\boldsymbol{w} \in H^{1}(\Omega)^{2} \text { such that } \operatorname{div} \underline{\boldsymbol{\sigma}}(\boldsymbol{w}) \in L^{2}(\Omega)^{2}\right\} \\
& \equiv\left\{\boldsymbol{w} \in H^{1}(\Omega)^{2} /-V_{P}^{2} \nabla(\operatorname{div} \boldsymbol{w})+V_{S}^{2} \operatorname{curl}(\operatorname{curl} \boldsymbol{w}) \in L^{2}(\Omega)^{2}\right\},
\end{aligned}
$$

where the second line comes from using (4). This space is a Hilbert space for the norm:

$$
\|\boldsymbol{w}\|_{\boldsymbol{D}}^{2}=\|\boldsymbol{w}\|_{H^{1}(\Omega)}^{2}+\|\operatorname{div} \underline{\boldsymbol{\sigma}}(\boldsymbol{w})\|_{L^{2}(\Omega)}^{2} .
$$

Note that, by construction of $\boldsymbol{D}$ and definition of $\boldsymbol{V}$,

$$
\forall \boldsymbol{u} \in \boldsymbol{D}, \quad \mathcal{F} \boldsymbol{u}:=\left(V_{P}^{2} \operatorname{div} \boldsymbol{u},-V_{S}^{2} \operatorname{curl} \boldsymbol{u}\right) \in \boldsymbol{V}
$$

Next, we consider the closed subspace of $\boldsymbol{D}$ of vector fields that are orthogonal to the rigid displacements and satisfy the free boundary condition

$$
\boldsymbol{D}_{N}:=\left\{\boldsymbol{w} \in \boldsymbol{D} \cap \boldsymbol{H}_{R}^{1}(\Omega) \text { such that } \underline{\boldsymbol{\sigma}}(\boldsymbol{w}) \boldsymbol{n}=0 \text { on } \Gamma\right\},
$$

and finally the space

$$
\boldsymbol{V}_{N}:=\mathcal{F}\left(\boldsymbol{D}_{N}\right) \equiv\left\{\left(V_{P}^{2} \operatorname{div} \boldsymbol{w},-V_{S}^{2} \operatorname{curl} \boldsymbol{w}\right), \quad \boldsymbol{w} \in \boldsymbol{D}_{N}\right\}
$$

A first remarkable property of this space is given by the lemma

Lemma 3.12. The space $\boldsymbol{V}_{N}$ is a subspace of $\boldsymbol{V}_{0}$.

Proof. By (118) we already know that $\boldsymbol{V}_{N} \subset \boldsymbol{V}$. So we simply have to check the gauge conditions in $(72)$. Let $\boldsymbol{\psi} \in \boldsymbol{V}_{N}$, i.e.,

$$
\boldsymbol{\psi}=\left(V_{P}^{2} \operatorname{div} \boldsymbol{w},-V_{S}^{2} \operatorname{curl} \boldsymbol{w}\right), \quad \boldsymbol{w} \in \boldsymbol{D}_{N}
$$

The proof is essentially a matter of reproducing the computation in section 3.2 for proving (67), with $\boldsymbol{\psi}$ instead of $\partial_{t}^{2} \boldsymbol{\varphi}$ and $\boldsymbol{w}$ instead of $\boldsymbol{v}$. Simply note that $\underline{\boldsymbol{\sigma}}(\boldsymbol{w}) \boldsymbol{n}=\mathbf{0}$ on $\Gamma$ follows from $\boldsymbol{w} \in \boldsymbol{D}_{N}$ while the equivalent of $(66)$ is nothing but (121). Then we obtain the equivalent of (67), namely,

$$
\partial_{\tau} w_{1}=\frac{1}{2 V_{S}^{2}} \boldsymbol{\psi} \cdot \boldsymbol{\tau}, \quad \partial_{\tau} w_{2}=-\frac{1}{2 V_{S}^{2}} \boldsymbol{\psi} \cdot \boldsymbol{n}, \quad \text { on } \Gamma
$$

Finally, the gauge conditions are simply obtained by integrating the above equalities on $\Gamma$.

We shall use later another nice property of the space $\boldsymbol{V}_{N}$ :

Lemma 3.13. For any $\boldsymbol{\psi}=\left(\psi_{P}, \psi_{S}\right) \in \boldsymbol{V}_{N}, \nabla \psi_{P}+\operatorname{curl} \psi_{S} \in \boldsymbol{L}_{R}^{2}(\Omega)$. 
Proof. Let $\boldsymbol{\psi} \in \boldsymbol{V}_{N}$. Then, $\boldsymbol{\psi}=\left(V_{P}^{2} \operatorname{div} \boldsymbol{w},-V_{S}^{2} \operatorname{curl} \boldsymbol{w}\right)$ with $\boldsymbol{w} \in \boldsymbol{D}_{N}$. By (4), $\operatorname{div} \underline{\boldsymbol{\sigma}}(\boldsymbol{w})=\rho\left(\nabla \psi_{P}+\operatorname{curl} \psi_{S}\right)$, that we multiply by any $\boldsymbol{w}_{R} \in \boldsymbol{R}(\Omega)$ and integrate over $\Omega$ to obtain, using Green's formula

$\int_{\Omega} \underline{\boldsymbol{\sigma}}(\boldsymbol{w}): \underline{\varepsilon}\left(\boldsymbol{w}_{R}\right) \mathrm{d} \boldsymbol{x}+\int_{\Gamma} \underline{\boldsymbol{\sigma}}(\boldsymbol{w}) \boldsymbol{n} \cdot \boldsymbol{w}_{R} \mathrm{~d} \gamma=-\rho \int_{\Omega}\left(\nabla \psi_{P}+\operatorname{curl} \psi_{S}\right) \cdot \boldsymbol{w}_{R} \mathrm{~d} \boldsymbol{x}=0$,

since $\underline{\varepsilon}\left(\boldsymbol{w}_{R}\right)=0$ and $\underline{\boldsymbol{\sigma}}(\boldsymbol{w}) \boldsymbol{n}=0$ for $\boldsymbol{w} \in \boldsymbol{D}_{N}$.

Now, we remark that, thanks to the property (58) (see Lemma 3.1) and Lemma 3.12, the vector of potentials $\varphi(\cdot, t)$ belongs to $\boldsymbol{V}_{N}$ for all $t \geq 0$. This implies that the space $\boldsymbol{V}_{N}$ satisfies the requirement (108). Next we prove that the space $\boldsymbol{V}_{N}$ satisfies the requirement (109).

Theorem 3.14. The bilinear form $m(\cdot, \cdot)$ is positive definite in the space $\boldsymbol{V}_{N}$. Furthermore, there exists $\mathcal{C}>0$, only depending on $\Omega, \lambda, \mu$ and $\rho$ such that

$$
m(\boldsymbol{\psi}, \boldsymbol{\psi}) \geq C \int_{\Omega}|\boldsymbol{\psi}|^{2} d \boldsymbol{x}, \quad \forall \boldsymbol{\psi} \in \boldsymbol{V}_{N} .
$$

Proof. Let $\boldsymbol{\psi}=\left(\psi_{P}, \psi_{S}\right) \in \boldsymbol{V}_{N}$, i.e., $\boldsymbol{\psi}=\left(V_{P}^{2} \operatorname{div} \boldsymbol{w},-V_{S}^{2} \operatorname{curl} \boldsymbol{w}\right), \boldsymbol{w} \in \boldsymbol{D}_{N}$. Applying the identity (38)

$$
\int_{\Omega}|\underline{\varepsilon}(\boldsymbol{w})|^{2} \mathrm{~d} \boldsymbol{x}=\int_{\Omega}|\operatorname{div} \boldsymbol{w}|^{2} \mathrm{~d} \boldsymbol{x}+\frac{1}{2} \int_{\Omega}|\operatorname{curl} \boldsymbol{w}|^{2} \mathrm{~d} \boldsymbol{x}-2 \int_{\Gamma} w_{2} \partial_{\boldsymbol{\tau}} w_{1} \mathrm{~d} \boldsymbol{\gamma}
$$

so that we deduce from (3) that

$$
\begin{aligned}
\int_{\Omega} \underline{\boldsymbol{\sigma}}(\boldsymbol{w}): \underline{\boldsymbol{\varepsilon}}(\boldsymbol{w}) \mathrm{d} \boldsymbol{x} & =(\lambda+2 \mu) \int_{\Omega}|\operatorname{div} \boldsymbol{w}|^{2} \mathrm{~d} \boldsymbol{x}+\mu \int_{\Omega}|\operatorname{curl} \boldsymbol{w}|^{2} \mathrm{~d} \boldsymbol{x} \\
& -4 \mu \int_{\Gamma} w_{2} \partial_{\boldsymbol{\tau}} w_{1} \mathrm{~d} \boldsymbol{\gamma} .
\end{aligned}
$$

Since $\lambda+2 \mu=\rho V_{P}^{2}$ and $\mu=\rho V_{S}^{2}$, we compute from the definition of $\boldsymbol{\psi}$ that

$$
(\lambda+2 \mu) \int_{\Omega}|\operatorname{div} \boldsymbol{w}|^{2} \mathrm{~d} \boldsymbol{x}+\mu \int_{\Omega}|\operatorname{curl} \boldsymbol{w}|^{2} \mathrm{~d} \boldsymbol{x}=\rho m_{\Omega}(\boldsymbol{\psi}, \boldsymbol{\psi}) .
$$

On the other hand, using 122 (see the proof of Lemma 3.12 we obtain

$$
-4 \mu \int_{\Gamma} w_{2} \partial_{\boldsymbol{\tau}} w_{1} \mathrm{~d} \boldsymbol{\gamma}=\rho m_{\Gamma}(\boldsymbol{\psi}, \boldsymbol{\psi})
$$

which, combined to the two previous equalities, gives

$$
m(\boldsymbol{\psi}, \boldsymbol{\psi})=\frac{1}{\rho} \int_{\Omega} \underline{\varepsilon}(\boldsymbol{w}): \underline{\boldsymbol{\sigma}}(\boldsymbol{w}) \mathrm{d} \boldsymbol{x} .
$$

Therefore, since, by (3) again,

$$
\underline{\boldsymbol{\sigma}}(\boldsymbol{w}): \underline{\boldsymbol{\varepsilon}}(\boldsymbol{w})=\lambda \operatorname{div}(\boldsymbol{w})^{2}+2 \mu|\underline{\boldsymbol{\varepsilon}}(\boldsymbol{w})|^{2} \geq 2 \mu|\underline{\boldsymbol{\varepsilon}}(\boldsymbol{w})|^{2},
$$


we get

$$
m(\boldsymbol{\psi}, \boldsymbol{\psi}) \geq 2 V_{S}^{2} \int_{\Omega}|\underline{\varepsilon}(\boldsymbol{w})|^{2} \mathrm{~d} \boldsymbol{x} .
$$

In the space $\boldsymbol{D}_{N} \subset \boldsymbol{H}_{R}^{1}(\Omega)$, we can use the Korn's inequality 61 to obtain

$$
\int_{\Omega}|\underline{\boldsymbol{\varepsilon}}(\boldsymbol{w})|^{2} \mathrm{~d} \boldsymbol{x} \geq 2 \mathcal{C}_{\Omega} V_{S}^{2} \int_{\Omega}|\nabla \boldsymbol{w}|^{2} \mathrm{~d} \boldsymbol{x} .
$$

This allows us to conclude since, obviously, from the definition of $\boldsymbol{\psi}$,

$$
\int_{\Omega}|\nabla \boldsymbol{w}|^{2} \mathrm{~d} \boldsymbol{x} \geq \widetilde{\mathcal{C}} \int_{\Omega}|\boldsymbol{\psi}|^{2} \mathrm{~d} \boldsymbol{x}
$$

with $\widetilde{\mathcal{C}}$ only depending on $V_{P}$ and $V_{S}$.

At this level, we have identified a good space $\boldsymbol{V}_{N}$ satisfying the requirements at the beginning of this section. However, the definition of this space $(120)$ is quite theoretical and implicit and thus rather hard to use it numerically: in particular, it refers to displacement fields, which we want precisely to avoid. The goal of the next section is to give a more suitable characterization of $\boldsymbol{V}_{N}$.

\subsubsection{A characterization of the new space}

Let $\Pi_{R}$ be the $L^{2}(\Omega)^{2}$ orthogonal projection onto the space $\boldsymbol{L}_{R}^{2}(\Omega) \equiv \boldsymbol{R}(\Omega)^{\perp}$. Given $\boldsymbol{\psi} \in \boldsymbol{V}$, let us denote $\boldsymbol{w}_{\star}:=\mathcal{S}_{N} \boldsymbol{\psi}$ the solution of the following elastostatic problem (whose well-posedness follows from Fredholm alternative)

$$
\left\{\begin{array}{rr}
-\operatorname{div} \underline{\boldsymbol{\sigma}}\left(\boldsymbol{w}_{\star}\right)=-\rho \Pi_{R}\left(\nabla \psi_{P}+\operatorname{curl} \psi_{S}\right), & \text { in } \Omega, \\
\boldsymbol{w}_{\star} \in \boldsymbol{H}_{R}^{1}(\Omega), \quad \underline{\boldsymbol{\sigma}}\left(\boldsymbol{w}_{\star}\right) \boldsymbol{n}=\mathbf{0}, & \text { on } \Gamma .
\end{array}\right.
$$

The reader will easily verify that, by construction,

$$
\mathcal{S}_{N} \in \mathcal{L}(\boldsymbol{V} ; \boldsymbol{D}) \text { and } \mathcal{I} m\left(\mathcal{S}_{N}\right) \subset \boldsymbol{D}_{N}
$$

Even more we have the following result:

Lemma 3.15. The operator $\mathcal{S}_{N}$ is a left inverse of $\mathcal{F}$ restricted to the space $\boldsymbol{D}_{N}$. More precisely,

$$
\forall \boldsymbol{w} \in \boldsymbol{D}_{N}, \quad \boldsymbol{w}=\mathcal{S}_{N} \mathcal{F} \boldsymbol{w}
$$

As a consequence, the image of $\mathcal{S}_{N}$ coincides with the space $\boldsymbol{D}_{N}$.

Proof. Let $\boldsymbol{w} \in \boldsymbol{D}_{N} . \operatorname{As}-\operatorname{div} \underline{\boldsymbol{\sigma}}(\boldsymbol{w})=-\rho\left(\nabla \psi_{P}+\operatorname{curl} \psi_{S}\right)$ with $\boldsymbol{\psi}=\mathcal{F} \boldsymbol{w}$ (cf. the proof of Lemma 3.13), we deduce from Lemma 3.13 that

$$
\operatorname{div} \underline{\boldsymbol{\sigma}}(\boldsymbol{w}) \in \boldsymbol{L}_{R}^{2}(\Omega) .
$$

Then $\operatorname{div} \underline{\boldsymbol{\sigma}}(\boldsymbol{w})$ coincides with its own projection onto $\boldsymbol{L}_{R}^{2}(\Omega)$ and we can write

$$
\operatorname{div} \underline{\boldsymbol{\sigma}}(\boldsymbol{w})=\rho \Pi_{R}\left(\nabla \psi_{P}+\operatorname{curl} \psi_{S}\right) .
$$


Moreover, since $\boldsymbol{w} \in \boldsymbol{D}_{N}$ we have

$$
\boldsymbol{w} \in \boldsymbol{H}_{R}^{1}(\Omega), \quad \underline{\boldsymbol{\sigma}}(\boldsymbol{w}) \boldsymbol{n}=\mathbf{0} \quad \text { on } \Gamma .
$$

Finally, (125) and 124 prove that $\boldsymbol{w}=\mathcal{S}_{N} \boldsymbol{\psi}$, i.e., $\boldsymbol{w}=\mathcal{S}_{N} \mathcal{F} \boldsymbol{w}$. Of course, writing $\boldsymbol{w}=\mathcal{S}_{N} \mathcal{F} \boldsymbol{w}$ for any $\boldsymbol{w} \in \boldsymbol{D}_{N}$, proves that $\boldsymbol{D}_{N} \subset \mathcal{I} m \mathcal{S}_{N}$.

Since $\mathcal{F} \in \mathcal{L}(\boldsymbol{D}, \boldsymbol{V})$ we can define

$$
\mathcal{T}:=\mathcal{F} \circ \mathcal{S}_{N} \in \mathcal{L}(\boldsymbol{V}), \quad \text { and } \quad \mathcal{I} m \mathcal{T}=\boldsymbol{V}_{N},
$$

where the equality derives from the definition of $\boldsymbol{V}_{N}$ and Lemma 3.15. We summarize in Figure 6 the images and preimages of the operator introduced so far, i.e. $\mathcal{F}, \mathcal{S}_{N}$ and $\mathcal{T}$.

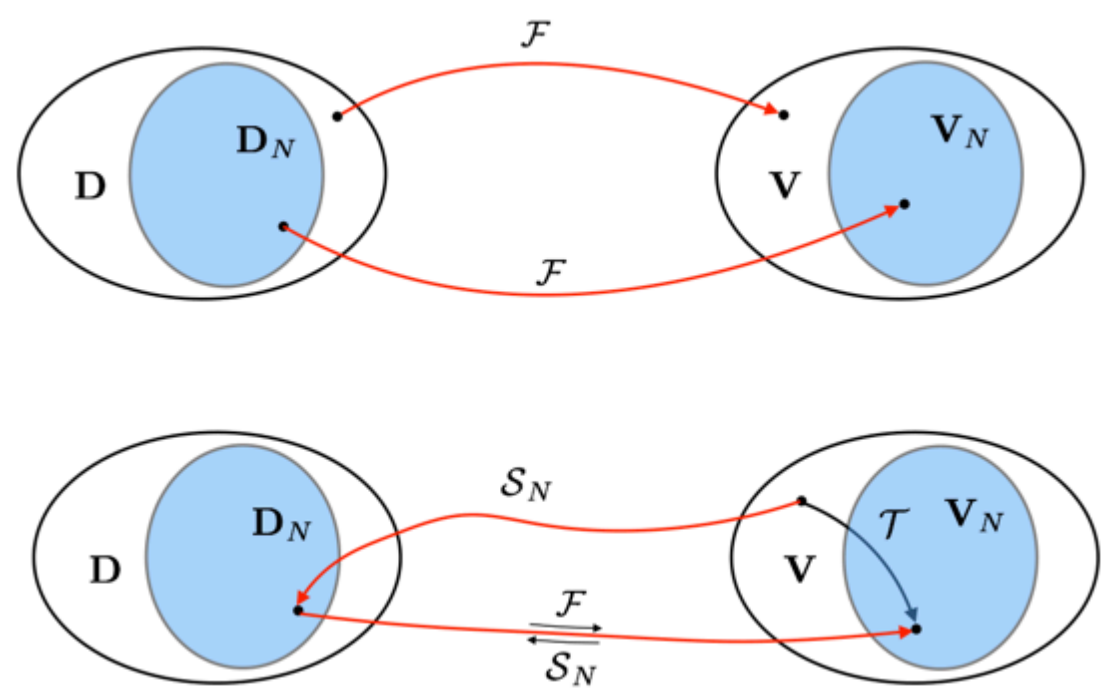

Figure 6: Representation of the images and pre-images of the operator $\mathcal{F}, \mathcal{S}_{N}$ and $\mathcal{T}$.

Note that, by definition of $\mathcal{S}_{N}$ (see 123) and $\mathcal{F}$ (118)

$$
\mathcal{T} \boldsymbol{\psi}=\left(V_{P}^{2} \operatorname{div} \boldsymbol{w}_{\star},-V_{S}^{2} \operatorname{curl} \boldsymbol{w}_{\star}\right) \text { where } \boldsymbol{w}_{\star} \text { is the solution of } 123 \text {. }
$$

A straighforward, but important, consequence of Lemma 3.15 is that $\mathcal{T}$ is a projector into $\boldsymbol{V}_{N}$. Indeed,

$$
\forall \boldsymbol{\psi} \in \boldsymbol{V}, \quad \mathcal{T}^{2} \boldsymbol{\psi}=\left(\mathcal{F} \mathcal{S}_{N}\right)\left(\mathcal{F} \mathcal{S}_{N}\right) \boldsymbol{\psi}=\mathcal{F}\left(\mathcal{S}_{N} \mathcal{F}\right) \mathcal{S}_{N} \psi
$$

Since $\mathcal{S}_{N} \boldsymbol{\psi} \in \boldsymbol{D}_{N}$, by Lemma $3.15 .\left(\mathcal{S}_{N} \mathcal{F}\right) \mathcal{S}_{N} \boldsymbol{\psi}=\mathcal{S}_{N} \boldsymbol{\psi}$ and thus

$$
\forall \boldsymbol{\psi} \in \boldsymbol{V}, \quad \mathcal{T}^{2} \boldsymbol{\psi}=\mathcal{F} \mathcal{S}_{N} \boldsymbol{\psi}=\mathcal{T} \boldsymbol{\psi} \quad \Longleftrightarrow \quad \mathcal{T}=\mathcal{T}^{2} .
$$


As for any projector, we can write the direct sum

$$
\boldsymbol{V}=\operatorname{ker} \mathcal{T} \oplus \mathcal{I} m \mathcal{T}=\operatorname{ker} \mathcal{T} \oplus \boldsymbol{V}_{N}
$$

Contrary to $\boldsymbol{V}$ or $\boldsymbol{V}_{N}$, it is possible to give an explicit description of the space ker $\mathcal{T}$ which is completely independent of the spaces $\boldsymbol{D}$ or $\boldsymbol{D}_{N}$. More precisely,

Lemma 3.16. The kernel of the operator $\mathcal{T}$ is characterized by

$$
\operatorname{ker} \mathcal{T}=\left\{\boldsymbol{\psi}^{o}=\left(\psi_{P}^{o}, \psi_{S}^{o}\right) \in \boldsymbol{V} / \nabla \psi_{P}^{o}+\operatorname{curl} \psi_{S}^{o} \in \boldsymbol{R}(\Omega)\right\} .
$$

Proof. Let $\varphi \in \operatorname{ker} \mathcal{T}$, and $\boldsymbol{w}_{\star}=\mathcal{S}_{N} \varphi$, solution of 123). Then $\mathcal{T} \varphi=0$ means that $\operatorname{div} \boldsymbol{w}_{\star}=\operatorname{curl} \boldsymbol{w}_{\star}=0$, thus $\operatorname{div} \boldsymbol{\sigma}\left(\boldsymbol{w}_{\star}\right)=\mathbf{0}$ by (4). In consequence of (123), $\Pi_{R}\left(\nabla \varphi_{P}+\operatorname{curl} \varphi_{S}\right)=\mathbf{0}$. The reverse implication is trivial.

The decomposition in 130 is not orthogonal in the classical sense but orthogonal with respect to $m(\cdot, \cdot)$ in the sense of the following theorem.

Theorem 3.17. We have $\boldsymbol{V}_{N}=(\operatorname{ker} \mathcal{T})^{\perp, m}$ where

$$
(\operatorname{ker} \mathcal{T})^{\perp, m}:=\left\{\boldsymbol{\psi} \in \boldsymbol{V} / \forall \boldsymbol{\psi}^{o} \in \operatorname{ker} \mathcal{T}, m\left(\boldsymbol{\psi}, \boldsymbol{\psi}^{o}\right)=0\right\}
$$

Proof. Step 1: $\boldsymbol{V}_{N} \subset(\operatorname{ker} \mathcal{T})^{\perp, m}$.

Let $\boldsymbol{\varphi}=\left(\varphi_{P}, \varphi_{S}\right) \in \boldsymbol{V}_{N}$ and $\boldsymbol{\psi}^{o}=\left(\psi_{P}^{o}, \psi_{S}^{o}\right) \in \operatorname{ker} \mathcal{T}$. We know that, by definition of $\boldsymbol{V}_{N}, \boldsymbol{\varphi}=\left(V_{P}^{2} \operatorname{div} \boldsymbol{w},-V_{S}^{2} \operatorname{curl} \boldsymbol{w}\right)$ with $\boldsymbol{w} \in \boldsymbol{D}_{N}$. Let us compute $m(\boldsymbol{\varphi}, \boldsymbol{\psi})=m_{\Omega}\left(\boldsymbol{\varphi}, \boldsymbol{\psi}^{o}\right)+m_{\Gamma}\left(\boldsymbol{\varphi}, \boldsymbol{\psi}^{o}\right)$. We have, by Green's formulas,

$$
\begin{aligned}
& \frac{1}{V_{P}^{2}} \int_{\Omega} \varphi_{P} \psi_{P}^{o} \mathrm{~d} \boldsymbol{x}=\int_{\Omega} \operatorname{div} \boldsymbol{w} \psi_{P}^{o} \mathrm{~d} \boldsymbol{x}=-\int_{\Omega} \boldsymbol{w} \cdot \nabla \psi_{P}^{o} \mathrm{~d} \boldsymbol{x}+\int_{\Gamma}(\boldsymbol{w} \cdot \boldsymbol{n}) \psi_{P}^{o} \mathrm{~d} \gamma, \\
& \frac{1}{V_{S}^{2}} \int_{\Omega} \varphi_{S} \psi_{S}^{o} \mathrm{~d} \boldsymbol{x}=-\int_{\Omega} \operatorname{curl} \boldsymbol{w} \psi_{S}^{o} \mathrm{~d} \boldsymbol{x}=-\int_{\Omega} \boldsymbol{w} \cdot \operatorname{curl} \psi_{S}^{o} \mathrm{~d} \boldsymbol{x}+\int_{\Gamma}(\boldsymbol{w} \cdot \boldsymbol{\tau}) \psi_{S}^{o} \mathrm{~d} \gamma .
\end{aligned}
$$

Let us add the two equalities. Since by Lemma $3.16 \nabla \psi_{P}^{o}+\operatorname{curl} \psi_{S}^{o} \in \boldsymbol{R}(\Omega)$, while $\boldsymbol{w} \in \mathbf{D}_{N} \subset \mathbf{L}_{R}^{2}(\Omega)$, the volume integral vanishes and

$$
m_{\Omega}\left(\boldsymbol{\varphi}, \boldsymbol{\psi}^{o}\right)=\int_{\Gamma}\left((\boldsymbol{w} \cdot \boldsymbol{n}) \psi_{P}^{o}+(\boldsymbol{w} \cdot \boldsymbol{\tau}) \psi_{S}^{o}\right) \mathrm{d} \gamma
$$

According to the proof of Lemma 3.12 we can use 122 , with $\boldsymbol{\varphi}$ instead of $\boldsymbol{\psi}$, which gives, for come constants $C_{1}$ and $C_{2}$,

$$
\frac{1}{2 V_{S}^{2}} \mathcal{I}(\boldsymbol{\varphi} \cdot \boldsymbol{\tau})=w_{1}+\mathcal{C}_{1}, \quad-\frac{1}{2 V_{S}^{2}} \mathcal{I}(\boldsymbol{\varphi} \cdot \boldsymbol{n})=w_{2}+C_{2}, \quad \text { on } \Gamma .
$$

Substituting (133) into the expression (76) of $m_{\Gamma}(\cdot, \cdot)$, we get, using the gauge conditions for $\boldsymbol{\psi}^{\circ}$,

$$
m_{\Gamma}\left(\boldsymbol{\varphi}, \boldsymbol{\psi}^{o}\right)=-\int_{\Gamma}\left(w_{1}\left(\boldsymbol{\psi}^{o} \cdot \boldsymbol{\tau}\right)+w_{2}\left(\boldsymbol{\psi}^{o} \cdot \boldsymbol{n}\right)\right) \mathrm{d} \gamma .
$$


Finally, adding (132) and (134) gives $m\left(\boldsymbol{\varphi}, \boldsymbol{\psi}^{\circ}\right)=0$ thanks to the identity (74).

Step 2: $(\operatorname{ker} \mathcal{T})^{\perp, m} \subset \boldsymbol{V}_{N}$.

Let $\boldsymbol{\varphi} \in(\operatorname{ker} \mathcal{T})^{\perp, m}$, i.e. such that $m\left(\boldsymbol{\varphi}, \boldsymbol{\psi}^{o}\right)=0$ for all $\boldsymbol{\psi}^{o} \in \operatorname{ker} \mathcal{T}$. Let $\boldsymbol{\psi} \in \boldsymbol{V}$. Using 130, we decompose $\varphi, \psi$ as:

$$
\boldsymbol{\varphi}=\varphi^{o}+\boldsymbol{\varphi}_{N}, \quad \boldsymbol{\psi}=\boldsymbol{\psi}^{o}+\boldsymbol{\psi}_{N}, \quad\left(\boldsymbol{\varphi}^{o}, \boldsymbol{\psi}^{o}\right) \in(\operatorname{ker} \mathcal{T})^{2}, \quad\left(\boldsymbol{\varphi}_{N}, \boldsymbol{\psi}_{N}\right) \in \boldsymbol{V}_{N}^{2}
$$

Our goal is to prove that $\varphi^{o}=0$. Note that by step 1 ,

$$
m\left(\boldsymbol{\varphi}^{o}, \boldsymbol{\psi}_{N}\right)=m\left(\boldsymbol{\varphi}_{N}, \boldsymbol{\psi}^{o}\right)=0 .
$$

Therefore, we compute

$$
m\left(\boldsymbol{\varphi}^{o}, \boldsymbol{\psi}\right)=m\left(\boldsymbol{\varphi}^{o}, \boldsymbol{\psi}^{o}\right)+m\left(\boldsymbol{\varphi}^{o}, \boldsymbol{\psi}_{N}\right)=m\left(\boldsymbol{\varphi}^{o}, \boldsymbol{\psi}^{o}\right) .
$$

Next, since $\varphi^{o}=\boldsymbol{\varphi}-\boldsymbol{\varphi}_{N}$ and $\boldsymbol{\varphi} \in(\operatorname{ker} \mathcal{T})^{\perp, m}$,

$$
m\left(\boldsymbol{\varphi}^{o}, \boldsymbol{\psi}\right)=m\left(\boldsymbol{\varphi}, \boldsymbol{\psi}^{o}\right)-m\left(\boldsymbol{\varphi}_{N}, \boldsymbol{\psi}^{o}\right) \underset{(135)}{=} m\left(\boldsymbol{\varphi}, \boldsymbol{\psi}^{o}\right)=0 .
$$

Thus $m\left(\boldsymbol{\varphi}^{o}, \boldsymbol{\psi}\right)=0, \forall \boldsymbol{\psi} \in \boldsymbol{V}$. In particular, for $\boldsymbol{\psi} \in(\mathcal{D}(\Omega))^{2} \subset \boldsymbol{V}$,

$$
m_{\Omega}\left(\boldsymbol{\varphi}^{o}, \boldsymbol{\psi}\right)=m\left(\boldsymbol{\varphi}^{o}, \boldsymbol{\psi}\right)=0 .
$$

We conclude by density of $\mathcal{D}(\Omega)^{2}$ in $L^{2}(\Omega)^{2}$ that $\varphi^{o}=\mathbf{0}$, thus $\boldsymbol{\varphi} \in \boldsymbol{V}_{N}$.

\subsubsection{A first stabilized mixed formulation}

In this section, we are going to exploit Theorem 3.17 namely

$$
\boldsymbol{\varphi} \in \boldsymbol{V}_{N} \Longleftrightarrow \boldsymbol{\varphi} \in \boldsymbol{V} \text { and } m(\boldsymbol{\varphi}, \boldsymbol{\psi})=0, \quad \forall \boldsymbol{\psi} \in \operatorname{ker} \mathcal{T}
$$

by reinterpreting the last condition as an equality constraint on $\varphi$. As it is usual for treating equality constraints (see [19, 20, 21]), we are going to introduce a Lagrange multiplier in the space ker $\mathcal{T}$. This leads us to write the following mixed problem

$$
\begin{cases}\text { Find } \left.\left(\boldsymbol{\varphi}(t), \boldsymbol{\varphi}^{o}(t)\right): \mathbb{R}^{+} \longrightarrow \boldsymbol{V} \times \operatorname{ker} \mathcal{T} \text { satisfying } 71\right) & \text { and } \\
\begin{array}{ll}
\frac{\mathrm{d}^{2}}{\mathrm{~d} t^{2}} m(\boldsymbol{\varphi}(t), \boldsymbol{\psi})+a(\boldsymbol{\varphi}(t), \boldsymbol{\psi})+m\left(\boldsymbol{\varphi}^{o}(t), \boldsymbol{\psi}\right)=l(t, \boldsymbol{\psi}), & \forall \boldsymbol{\psi} \in \boldsymbol{V}, \\
m\left(\boldsymbol{\varphi}(t), \boldsymbol{\psi}^{o}\right)=0, & \forall \boldsymbol{\psi}^{o} \in \operatorname{ker} \mathcal{T} .
\end{array}\end{cases}
$$

Then we have the following equivalence theorem between the above mixed problem and the variational problem 108 posed in the space $\boldsymbol{V}_{N}$.

Theorem 3.18. The problem (136) admits a unique solution given by $(\boldsymbol{\varphi}(t), \mathbf{0})$ where $\varphi(t)$ is the solution of the problem (108). 
Proof. Let $\varphi(t)$ be the solution of (108). First, it is clear that it satisfies the second equation in 136 since $\boldsymbol{\varphi}(t) \in \boldsymbol{V}_{N}$ and $\boldsymbol{V}_{N}=(\operatorname{ker} \mathcal{T})^{\perp, m}$ (Theorem 3.17). This also implies that

$$
\frac{\mathrm{d}^{2}}{\mathrm{~d} t^{2}} m\left(\boldsymbol{\varphi}(t), \boldsymbol{\psi}^{o}\right)=0, \quad \forall \boldsymbol{\psi}^{o} \in \operatorname{ker} \mathcal{T} .
$$

On the other hand, by definition (31) of $a(\cdot, \cdot)$, we have

$$
a\left(\boldsymbol{\varphi}(t), \boldsymbol{\psi}^{o}\right)=\int_{\Omega}\left(\nabla \varphi_{P}(t)+\operatorname{curl} \varphi_{S}(t)\right) \cdot\left(\nabla \psi_{P}^{o}+\operatorname{curl} \psi_{S}^{o}\right) \mathrm{d} \boldsymbol{x} .
$$

By Lemma 3.16, we know that $\nabla \psi_{P}^{o}+\operatorname{curl} \psi_{S}^{o} \in \boldsymbol{R}(\Omega)$. Then, since $\boldsymbol{\varphi}(t) \in \boldsymbol{V}_{N}$ we deduce $a\left(\boldsymbol{\varphi}(t), \boldsymbol{\psi}^{o}\right)=0$ from Lemma 3.13 . Finally, thanks to the assumption (60) on the source term, defined by $\left(9,190\right.$, we have $l\left(t, \boldsymbol{\psi}^{o}\right)=0$. Thus, we conclude that

$$
\frac{\mathrm{d}^{2}}{\mathrm{~d} t^{2}} m\left(\boldsymbol{\varphi}(t), \boldsymbol{\psi}^{o}\right)+a\left(\boldsymbol{\varphi}(t), \boldsymbol{\psi}^{o}\right)=l\left(t, \boldsymbol{\psi}^{o}\right), \quad \forall \boldsymbol{\psi}^{o} \in \operatorname{ker} \mathcal{T}
$$

Since $\varphi$ is the solution 108 , we also have

$$
\frac{\mathrm{d}^{2}}{\mathrm{~d} t^{2}} m(\boldsymbol{\varphi}(t), \boldsymbol{\psi})+a(\boldsymbol{\varphi}(t), \boldsymbol{\psi})=l(t, \boldsymbol{\psi}), \quad \forall \boldsymbol{\psi} \in \boldsymbol{V}_{N},
$$

and then, by linearity and using the decomposition 130 of $\boldsymbol{V}$, we obtain the above equality also for all $\boldsymbol{\psi} \in \boldsymbol{V}$ which is nothing but the first equation in 136 with $\boldsymbol{\varphi}^{o}=\mathbf{0}$. We thus have proven that $(\boldsymbol{\varphi}(t), \mathbf{0})$ is solution of 136.

It remains to prove the uniqueness of solutions of $(136)$. Let $\left(\varphi, \varphi^{\circ}\right)$ be a solution of (136) with $l(\cdot, \cdot)=0$. Then, by restricting the test function $\boldsymbol{\psi}$ in the second line of (136) to $\boldsymbol{\psi} \in \boldsymbol{V}_{N}$, using again the m-orthogonality of $\boldsymbol{V}_{N}$ and $\operatorname{ker} \mathcal{T}$, we deduce that $\varphi$ is the solution of (108) with $l(\cdot, \cdot)=0$. Thus $\varphi=0$. We then deduce from the second line in (136) again that

$$
m\left(\boldsymbol{\varphi}^{o}(t), \boldsymbol{\psi}\right)=0, \quad \forall \boldsymbol{\psi} \in \boldsymbol{V},
$$

which leads to $\varphi^{\circ}=\mathbf{0}$ due to the injectivity property (80) (see Lemma 3.5).

The reader which is not familiar with mixed variational formulation could be surprised that we introduce an additionnal unknown that we know is $\mathbf{0}$. All the interest of this new formulation is when Galerkin discretization is concerned: after discretization we get a stable problem and the discrete approximation of the unknown $\varphi^{\circ}$ is no longer $\mathbf{0}$ (see Remark 3.23).

\subsubsection{Characterization of the multipliers space $\operatorname{ker} \mathcal{T}$}

Even though the mixed variational problem $(136)$ is nicer than 108$)$ in the sense that any reference to displacement fields is removed, it is still not completely 
satisfactory for finite element approximation since we need a priori to construct a Galerkin approximation space for $\operatorname{ker} \mathcal{T}$. In order to work around this problem we are going to characterize the space $\operatorname{ker} \mathcal{T}$ up to an explicit three dimensional subspace (see Lemma 3.19) as the image by an explicit mapping $\mathcal{E}$ of a space $M$ of functions along the boundary $\Gamma$ (see (143)). This is then satisfactory from the numerical point of view because it is easy to approximate the space $M$ with finite elements (defined on the boundary $\Gamma$ ) while the operator $\mathcal{E}$ is easy to approximate numerically. This will result in an alternative reformulation of the mixed problem which will be given in section 3.3.5. We first begin with a lemma.

Lemma 3.19. The space $\operatorname{ker} \mathcal{T}$ can be decomposed as the direct sum

$$
\operatorname{ker} \mathcal{T}=\boldsymbol{K}_{R} \oplus \boldsymbol{K}_{0}
$$

where $\boldsymbol{K}_{0}$ is the closed space of $\boldsymbol{V}$ of the so-called harmonic fields defined by

$$
\begin{aligned}
\boldsymbol{K}_{0} & =\left\{\boldsymbol{\psi}=\left(\psi_{P}, \psi_{S}\right) \in \boldsymbol{V} / \nabla \psi_{P}+\operatorname{curl} \psi_{S}=\mathbf{0}\right\} \\
& \equiv\{\boldsymbol{\psi} \in \boldsymbol{V} / \operatorname{div} \boldsymbol{\psi}=\operatorname{curl} \boldsymbol{\psi}=0\}
\end{aligned}
$$

and $\boldsymbol{K}_{R}$ is the 3 dimensional space $\boldsymbol{K}_{R}=\operatorname{span}\left\{\varphi_{1}, \varphi_{2}, \varphi_{3}\right\}$ where

$$
\boldsymbol{\varphi}_{1}=\frac{1}{2}\left(x_{1}, x_{2}\right)^{t}, \quad \boldsymbol{\varphi}_{2}=\frac{1}{2}\left(x_{2},-x_{1}\right)^{t}, \quad \boldsymbol{\varphi}_{3}=\frac{1}{2}\left(0, x_{1}^{2}+x_{2}^{2}\right)^{t} .
$$

Proof. First, we easily check that

$$
\left(\begin{array}{c}
\operatorname{div} \varphi_{1} \\
-\operatorname{curl} \varphi_{1}
\end{array}\right)=\left(\begin{array}{l}
1 \\
0
\end{array}\right), \quad\left(\begin{array}{c}
\operatorname{div} \varphi_{2} \\
-\operatorname{curl} \varphi_{2}
\end{array}\right)=\left(\begin{array}{l}
0 \\
1
\end{array}\right), \quad\left(\begin{array}{c}
\operatorname{div} \varphi_{3} \\
-\operatorname{curl} \varphi_{3}
\end{array}\right)=\left(\begin{array}{c}
x_{2} \\
-x_{1}
\end{array}\right)
$$

By (55), if $\boldsymbol{\psi} \in \operatorname{ker} \mathcal{T}$, there exist $\left(a_{1}, a_{2}, b\right) \in \mathbb{R}$ such that

$$
\left(\begin{array}{c}
\operatorname{div} \boldsymbol{\psi} \\
-\operatorname{curl} \boldsymbol{\psi}
\end{array}\right)=a_{1}\left(\begin{array}{l}
1 \\
0
\end{array}\right)+a_{2}\left(\begin{array}{l}
0 \\
1
\end{array}\right)+b\left(\begin{array}{c}
x_{2} \\
-x_{1}
\end{array}\right)
$$

Then $\boldsymbol{\psi}-\left(a_{1} \boldsymbol{\varphi}_{1}+a_{2} \boldsymbol{\varphi}_{2}+b \boldsymbol{\varphi}_{3}\right) \in \mathbf{K}_{0}$. To conclude is suffices to remark, using the formulae 140 , that $\mathbf{K}_{R} \cap \mathbf{K}_{0}=\emptyset$.

Next, we show that the space $\boldsymbol{K}_{0}$ can be identified with a space of functions defined on the boundary $\Gamma$. To do so, we introduce the classical normal trace map

$$
\gamma \in \mathcal{L}\left(H(\operatorname{div}, \Omega), H^{-\frac{1}{2}}(\Gamma)\right), \quad \gamma: \boldsymbol{\varphi} \longrightarrow \boldsymbol{\varphi} \cdot \boldsymbol{n}_{\mid \Gamma}
$$

Theorem 3.20. The map $\gamma$ is an isomorphism from $\boldsymbol{K}_{0}$ onto

$$
M:=\left\{\nu \in H^{-\frac{1}{2}}(\Gamma) / \int_{\Gamma} \nu \mathrm{d} \gamma=0\right\}
$$


Proof. The fact that $\gamma \boldsymbol{\varphi} \in M$ for $\boldsymbol{\varphi} \in \boldsymbol{K}_{0}$ follows from Green's formula

$$
\left.\int_{\Gamma} \boldsymbol{\varphi} \cdot \boldsymbol{n} \mathrm{d} \gamma=\int_{\Omega} \operatorname{div} \boldsymbol{\varphi} \mathrm{d} \gamma=0, \quad \text { since } \operatorname{div} \boldsymbol{\varphi}=0 \text { (second line of } 138\right) \text { ). }
$$

To conclude, it suffices to show that for any $\nu \in M$, there exists a unique $\boldsymbol{\varphi} \in \boldsymbol{K}_{0}$ such that $\gamma \boldsymbol{\varphi}=\nu$. For the existence, let $p$ be the unique solution of the Neumann problem (note that $\nu \in M$ yields the compatibility condition required for the unique solvability of this problem)

$$
\left\{\begin{aligned}
\text { Find } p & \in H^{1}(\Omega) / \mathbb{R} \text { such that } \\
-\Delta p & =0, \quad \text { in } \Omega \\
\partial_{\boldsymbol{n}} p & =\nu, \quad \text { in } \Gamma,
\end{aligned}\right.
$$

Then setting $\boldsymbol{\varphi}=\nabla p$ we have $\operatorname{div} \boldsymbol{\varphi}=\operatorname{curl} \boldsymbol{\varphi}=0$ and $\boldsymbol{\varphi} \cdot \boldsymbol{n}=\partial_{\boldsymbol{n}} p=\nu$ on $\Gamma$, hence $\varphi \in \boldsymbol{K}_{0}$.

For the uniqueness, we have simply to remark that $\operatorname{curl} \psi=0$ in $\Omega$ implies that $\boldsymbol{\psi}=\nabla q$, with $q \in H^{1}(\Omega) / \mathbb{R}$ (see Theorem 2.9 in [2]). Then if $\boldsymbol{\psi} \cdot \boldsymbol{n}=0$ on $\Gamma$ and $\operatorname{div} \boldsymbol{\psi}=0$ we have $\Delta q=0$ as well as $\partial_{\boldsymbol{n}} q=0$ therefore $q=0$ and $\boldsymbol{\psi}=\mathbf{0}$.

The above proof shows that the inverse of the map $\gamma$ is the lifting operator

$$
\mathcal{E} \in \mathcal{L}\left(M, \boldsymbol{K}_{0}\right),
$$

where $\mathcal{E} \mu:=\nabla p$, with $p$ the unique solution of 142. In other words, Theorem 3.20 can be rephrased as

$$
\boldsymbol{K}_{0}=\{\mathcal{E} \nu / \nu \in M\} .
$$

This characterization brings a new light on the absence of positivity of the bilinear form $m(\cdot, \cdot)$.

Corollary 3.21. For all $\boldsymbol{\psi} \in \boldsymbol{K}_{0}, m(\boldsymbol{\psi}, \boldsymbol{\psi}) \leq 0$.

Proof. From 143 we know that there exists $\nu$ such that $\boldsymbol{\psi}=\mathcal{E} \nu=\nabla p$ with $p$ the unique solution of 142 , then

$$
m(\nabla p, \nabla p)=\frac{1}{V_{P}^{2}} \int_{\Omega}\left|\partial_{1} p\right|^{2} \mathrm{~d} \boldsymbol{x}+\frac{1}{V_{S}^{2}} \int_{\Omega}\left|\partial_{2} p\right|^{2} \mathrm{~d} \boldsymbol{x}-\frac{1}{V_{S}^{2}} \int_{\Gamma} \mathcal{I}(\nabla p \cdot \boldsymbol{\tau}) \nabla p \cdot \boldsymbol{n} \mathrm{d} \boldsymbol{\gamma} .
$$

Since $\mathcal{I}(\nabla p \cdot \boldsymbol{\tau})=p+c$ for a constant $c$ and since $\nabla p \cdot \boldsymbol{n}=\nu$ has zero average along $\Gamma$

$$
\int_{\Gamma} \mathcal{I}(\nabla p \cdot \boldsymbol{\tau}) \nabla p \cdot \boldsymbol{n}=\int_{\Omega}|\nabla p|^{2} \mathrm{~d} \boldsymbol{x}+\int_{\Omega} p \Delta p \mathrm{~d} \boldsymbol{x}=\int_{\Omega}|\nabla p|^{2} \mathrm{~d} \boldsymbol{x} .
$$

Therefore combining the two previous equation we obtain

$$
m(\nabla p, \nabla p)=\left(\frac{1}{V_{P}^{2}}-\frac{1}{V_{S}^{2}}\right) \int_{\Omega}\left|\partial_{1} p\right|^{2} \mathrm{~d} \boldsymbol{x} \leq 0 .
$$


The result of Corollary 3.21 confirms that fact that the space $\boldsymbol{K}_{0}$ must be eliminated in the functional space in which the solution is sough.

Remark 3.22. Observe that if $\boldsymbol{\psi} \in \boldsymbol{K}_{0}$ then $m(\boldsymbol{\psi}, \boldsymbol{\psi})=0$ if and only if $\boldsymbol{\psi}=\nabla p$ with $\partial_{1} p=0$. Hence $p$ is a function of $x_{2}$ only. Since it is harmonic it can only be an affine function in $x_{2}$ and therefore $\boldsymbol{\psi}$ is proportional to $(0,1)^{t}$. It is also noticeable that the result of Corollary 3.21 uses explicitly the inequality $V_{P}>V_{S}$ which was not the case for Theorem 3.6.

\subsubsection{A second stabilized mixed formulation}

Using Lemma 3.19 and (143), we can now formulate a new mixed problem equivalent to (136), in which the Lagrange multiplier $\varphi^{\circ}$ is replaced by the $\left(\eta, \varphi_{R}\right)$ :

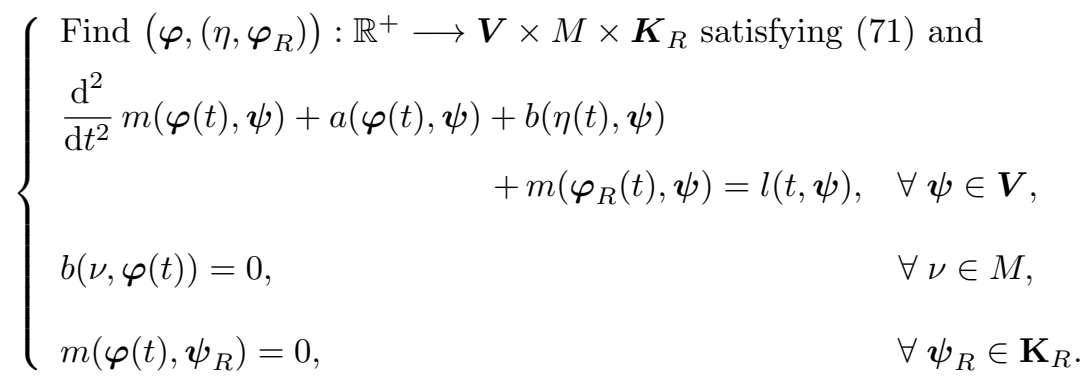

where the bilinear form $b(\cdot, \cdot)$ is defined by

$$
\forall(\nu, \boldsymbol{\psi}) \in M \times \boldsymbol{V}, \quad b(\nu, \boldsymbol{\psi}):=m(\mathcal{E} \nu, \boldsymbol{\psi}) .
$$

\subsubsection{Numerical approximation}

Introduction. The structure and notations of this section follows the structure of section 3.2.4 As previously explained, we consider as an approximation of $\boldsymbol{V}$ the space $\boldsymbol{V}_{h}=V_{P, h} \times V_{S, h}$ where $V_{P, h}$ and $V_{S, h}$ are approximations of $H^{1}(\Omega)$. The space $M$ is approximated by $M_{h}$ and $K_{R}$ is already finite dimensional and therefore no approximation is needed. The semi-discrete problem reads

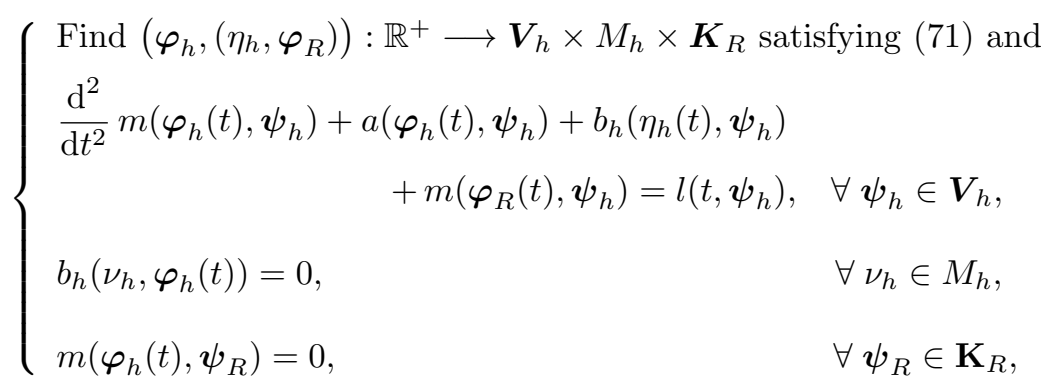

where $b_{h}(\cdot, \cdot):=m\left(\mathcal{E}_{h}(\cdot), \cdot\right)$ with $\mathcal{E}_{h}$ a discrete lifting operator that approximates $\mathcal{E}$ and which description is postponed to a forthcoming work. 
Remark 3.23. The last two equations of (146) show that it is equivalent to say that the solution $\varphi_{h}$ belongs to a subspace of $\boldsymbol{V}_{h}$ of co-dimension $\operatorname{dim} M_{h}+3$. This subspace is not a subspace of $\boldsymbol{V}_{N}$, in other words we realize a non-conform approximation of the space $\boldsymbol{V}_{N}$ and that is why the multiplier $\eta_{h}$ is not $\mathbf{0}$ (see remark 9.1 in [20] and [21] for similar situations).

The corresponding algebraic system of ODEs reads

$$
\left\{\begin{array}{l}
\mathbb{M}_{h} \frac{\mathrm{d}^{2} \boldsymbol{\Phi}_{h}}{\mathrm{~d} t^{2}}+\mathbb{A}_{h} \boldsymbol{\Phi}_{h}+\mathbb{B}_{h} \boldsymbol{H}_{h}+\mathbb{M}_{R, h} \boldsymbol{\Phi}_{R}=\boldsymbol{F}_{h}, \\
\mathbb{B}_{h}^{T} \boldsymbol{\Phi}_{h}=0 \\
\mathbb{M}_{R, h}^{T} \boldsymbol{\Phi}_{h}=0
\end{array}\right.
$$

where, $\boldsymbol{\Phi}_{h}, \boldsymbol{\Phi}_{R}$ and $\boldsymbol{H}_{h}$ are the vectors of degrees of freedom of $\boldsymbol{\varphi}_{h}, \boldsymbol{\varphi}_{R}, \eta_{h}$ respectively. Our objective being to show that the semi-discrete problem is stable we use the most simple time scheme one could use to solve (147), namely an explicit centered time discretization (i.e. the leap-frog scheme)

$$
\left\{\begin{array}{l}
\mathbb{M}_{h} \frac{\boldsymbol{\Phi}_{h}^{n+1}-2 \boldsymbol{\Phi}_{h}^{n}+\boldsymbol{\Phi}_{h}^{n-1}}{\Delta t^{2}}+\mathbb{A}_{h} \boldsymbol{\Phi}_{h}^{n}+\mathbb{B}_{h} \boldsymbol{H}_{h}^{n}+\mathbb{M}_{R, h} \boldsymbol{\Phi}_{R, h}^{n}=\boldsymbol{F}_{h}^{n}, \\
\mathbb{B}_{h}^{t} \boldsymbol{\Phi}_{h}^{n}=0 \\
\mathbb{M}_{R, h}^{t} \boldsymbol{\Phi}_{h}^{n}=0 .
\end{array}\right.
$$

At each iteration the matrix

$$
\left(\begin{array}{ccc}
\mathbb{M}_{h} / \Delta t^{2} & \mathbb{B}_{h} & \mathbb{M}_{R, h} \\
\mathbb{B}_{h}^{t} & 0 & 0 \\
\mathbb{M}_{R, h}^{t} & 0 & 0
\end{array}\right)
$$

has to be inverted. It is natural to think that a good choice of the approximation spaces $\boldsymbol{V}_{h}$ and $M_{h}$ would lead to an invertible matrix. The proof of such a result would rely on a discrete inf-sup condition. This is a fundamental question that we do not address in this paper, except in the next paragraph for the toy problem. In the more general configuration addressed at the end of this section, we will limit ourselves to describing the discretization spaces used in the implementation.

Going back to the toy problem. We come back to the domain 89). As already mentioned, this particular example does not fit completely in the theory developed since $\Omega$ is unbounded. A consequence of this is that no rigid motion exists in $L^{2}(\Omega)$ and the discrete variational formulation (147) is simplified. Some other minor modifications are involved. In particular, the space $M$ is defined as

$$
M:=\left\{\nu \in H^{-\frac{1}{2}}(-\pi, \pi) / \int_{-\pi}^{\pi} \nu\left(x_{2}\right) \mathrm{d} x_{2}=0\right\} .
$$


The lifting operator $\mathcal{E} \in \mathcal{L}(M, V)$ is defined as $\mathcal{E} \nu=\nabla p_{\nu}$ where $p_{\nu}$ is the unique solution of the variational problem

$$
\text { Find } p_{\nu} \in H_{p e r}^{1}(\Omega) \text { such that } \int_{\Omega} \nabla p_{\nu} \cdot \nabla q \mathrm{~d} \boldsymbol{x}=\int_{-\pi}^{\pi} \nu\left(x_{2}\right) q\left(x_{2}, 0\right) \mathrm{d} x_{2},
$$

where $H_{p e r}^{1}(\Omega)=\left\{p \in H^{1}(\Omega)\right.$ s.t. $\left.p\left(x_{1},-\pi\right)=p\left(x_{1}, \pi\right), x_{1} \in \mathbb{R}^{+}\right\}$. Both $p_{\nu}$ and $\mathcal{E} \nu$ and can be computed explicitly, using separation of variables and Fourier series in $x_{2}$. More precisely, assuming that

$$
\nu:=\sum_{\ell \neq 0} \nu_{\ell} e^{-i \ell x_{2}}
$$

one can easily show that

$$
p_{\nu}=\sum_{\ell \neq 0} \nu_{\ell} p^{\ell}\left(x_{1}\right) e^{-i \ell x_{2}} \quad \text { with } \quad p^{\ell}\left(x_{1}\right):=\frac{1}{|\ell|} e^{-|\ell| x_{1}}, \quad \forall \ell \neq 0,
$$

and, in consequence,

$$
\mathcal{E} \nu=\sum_{\ell \neq 0} \nu_{\ell} \mathcal{E}^{\ell}\left(x_{1}\right) e^{-i \ell x_{2}},
$$

with

$$
\mathcal{E}^{\ell}\left(x_{1}\right):=-\left(e^{-|\ell| x_{1}}, i \operatorname{sign}(\ell) e^{-|\ell| x_{1}}\right)^{t}, \quad \forall \ell \neq 0 .
$$

For the discretization, we keep the same space $\boldsymbol{V}_{h}$ as in section 3.2 .4 and we define

$$
M_{h} \equiv M_{L}:=\left\{\eta_{h}\left(x_{2}\right)=\sum_{\ell=-L}^{L} \eta^{\ell} e^{-i \ell x_{2}} \text { with } \eta^{0}=0\right\} .
$$

The mixed problem (146) can then be rewritten as a family of $2 L+1$ problems parametrized by $\ell$. For $\ell=0$, we simply solve (94) (see section 3.2.4) whereas, for $\ell \neq 0$, we have to solve the following problem (where the Lagrange multiplier is simply a scalar)

$$
\left\{\begin{array}{l}
\text { Find }\left(\boldsymbol{\varphi}_{h}^{\ell}, \eta^{\ell}\right): \mathbb{R}^{+} \longrightarrow \boldsymbol{V}_{h} \times \mathbb{R} \text { s.t. } \forall \boldsymbol{\psi}_{h}^{\ell} \in \boldsymbol{V}_{h}, \\
\frac{\mathrm{d}^{2}}{\mathrm{~d} t^{2}} m_{\ell}\left(\boldsymbol{\varphi}_{h}^{\ell}(t), \boldsymbol{\psi}_{h}^{\ell}\right)+a\left(\boldsymbol{\varphi}_{h}^{\ell}(t), \boldsymbol{\psi}_{h}^{\ell}\right)+\eta^{\ell} b_{\ell}\left(\boldsymbol{\psi}_{h}^{\ell}\right)=l_{\ell}\left(t, \boldsymbol{\psi}_{h}^{\ell}\right), \\
b_{\ell}\left(\boldsymbol{\varphi}_{h}^{\ell}\right)=0
\end{array}\right.
$$

where the linear form $b_{\ell}\left(\boldsymbol{\psi}_{h}^{\ell}\right):=m_{\ell}\left(\boldsymbol{\psi}_{h}^{\ell}, \mathcal{E}^{\ell}\right)$ is, according to 152 ,

$$
\begin{aligned}
b_{\ell}\left(\boldsymbol{\psi}_{h}^{\ell}\right) & =\frac{-1}{V_{P}^{2}} \oint_{\mathbb{R}^{+}} \psi_{P, h}^{\ell} \mathbf{e}_{\ell} \mathrm{d} x_{1}+\frac{i \operatorname{sign}(\ell)}{V_{S}^{2}} \oint_{\mathbb{R}^{+}} \psi_{S, h}^{\ell} \mathbf{e}_{\ell} \mathrm{d} x_{1} \\
& -\frac{i}{2 V_{S}^{2} \ell} \psi_{S, h}^{\ell}(0)+\frac{1}{2 V_{S}^{2}|\ell|} \psi_{P, h}^{\ell}(0),
\end{aligned}
$$


where we have set $\mathbf{e}_{\ell}\left(x_{1}\right)=e^{-|\ell| x_{1}}$.

Next we investigate the invertibility of the operator 149 (where the last column and line must not be taken into account since no rigid displacements exist in $L^{2}(\Omega)$ ). It is well known (see [22]) that it is sufficient to prove the inf-sup condition for the bilinear form $c_{\ell}\left(\eta, \varphi_{h}^{\ell}\right):=\eta b_{\ell}\left(\varphi_{h}^{\ell}\right)$ and the the coercivity of the bilinear form $m_{\ell}(\cdot, \cdot)$ on the kernel of $b_{\ell}(\cdot)$ (see Theorem 1.1 of [22]). The former being rather easy to be obtained in this case, we will focus on the latter. More precisely, we are going to prove that there exists $C>0$ independent of $h$ and $\ell$ such that for any $\varphi_{h}^{\ell}$ satisfying

$$
b_{\ell}\left(\varphi_{h}^{\ell}\right)=0 \quad \text { then } \quad m_{\ell}\left(\varphi_{h}^{\ell}, \boldsymbol{\varphi}_{h}^{\ell}\right)>C\left\|\varphi_{h}^{\ell}\right\|_{h}^{2} .
$$

This results is a discrete equivalent of the result of Theorem 3.14. It does not only show the invertibility of 149$)$ but also demonstrates the stability of the semi-discrete evolution problem (147). Indeed, this results from the control of the discrete energy

$$
E_{\ell}(t):=\frac{1}{2} m_{\ell}\left(\frac{\mathrm{d}}{\mathrm{d} t} \boldsymbol{\varphi}_{h}^{\ell}(t), \frac{\mathrm{d}}{\mathrm{d} t} \boldsymbol{\varphi}_{h}^{\ell}(t)\right)+\frac{1}{2} a_{l}\left(\boldsymbol{\varphi}_{h}^{\ell}(t), \boldsymbol{\varphi}_{h}^{\ell}(t)\right) .
$$

We are going to prove that 157 holds under some non degenerescence of the mesh, namely

$$
h L \leq 1 .
$$

Let us prove estimate (157). First of all, from 95 we obtain

$$
m_{\ell}\left(\boldsymbol{\varphi}_{h}^{\ell}, \boldsymbol{\varphi}_{h}^{\ell}\right)=\frac{1}{V_{P}^{2}}\left\|\varphi_{P, h}^{\ell}\right\|_{h}^{2}+\frac{1}{V_{S}^{2}}\left\|\varphi_{S, h}^{\ell}\right\|_{h}^{2}-\frac{1}{V_{S}^{2} \ell} \operatorname{Im}\left(\overline{\varphi_{P}^{\ell, 0}} \varphi_{S}^{\ell, 0}\right) .
$$

Next we obtain bounds for the last term on the right hand side. From the assumption in 157) we know that $i \overline{\varphi_{P}^{\ell, 0}} \cdot b_{\ell}\left(\varphi_{h}^{\ell}\right)=0$, i.e.,

$$
-\frac{\overline{\varphi_{P}^{\ell, 0}}}{V_{S}^{2} \ell} \varphi_{S}^{\ell, 0}=\frac{i}{V_{S}^{2}|\ell|}\left|\varphi_{P}^{\ell, 0}\right|^{2}-2 \overline{\varphi_{P}^{\ell, 0}} \oint_{\mathbb{R}^{+}}\left[\frac{i \varphi_{P, h}^{\ell}}{V_{P}^{2}}+\frac{\varphi_{S, h}^{\ell} \operatorname{sign}(\ell)}{V_{S}^{2}}\right] \mathbf{e}_{\ell} \mathrm{d} x_{1}
$$

which implies

$$
-\frac{1}{V_{S}^{2} \ell} \operatorname{Im}\left(\overline{\varphi_{P}^{\ell, 0}} \varphi_{S}^{\ell, 0}\right)=\frac{\left|\varphi_{P}^{\ell, 0}\right|^{2}}{V_{S}^{2}|\ell|}-2 \operatorname{Im}\left(\overline{\varphi_{P}^{\ell, 0}} \oint_{\mathbb{R}^{+}}\left[\frac{i \varphi_{P, h}^{\ell}}{V_{P}^{2}}+\frac{\varphi_{S, h}^{\ell} \operatorname{sign}(\ell)}{V_{S}^{2}}\right] \mathbf{e}_{\ell} \mathrm{d} x_{1}\right) .
$$

Thus, using Cauchy-Schwarz inequality and the definitions 93 , we get

$$
-\frac{1}{V_{S}^{2} \ell} \operatorname{Im}\left(\overline{\varphi_{P}^{\ell, 0}} \varphi_{S}^{\ell, 0}\right) \geq \frac{\left|\varphi_{P}^{\ell, 0}\right|^{2}}{V_{S}^{2}|\ell|}-2\left|\varphi_{P}^{\ell, 0}\right|\left(\frac{\left\|\varphi_{P, h}^{\ell}\right\|_{h}}{V_{P}^{2}}+\frac{\left\|\varphi_{S, h}^{\ell}\right\|_{h}}{V_{S}^{2}}\right)\left\|\mathbf{e}_{\ell}\right\|_{h} .
$$

Then by Young's inequality, we get that for any $\eta>0(Q \in\{P, S\})$

$$
2\left|\varphi_{P}^{\ell, 0}\right|\left\|\varphi_{Q, h}^{\ell}\right\|\left\|_{h}\right\| \mathbf{e}_{\ell}\left\|_{h} \leq \eta\left|\varphi_{P}^{\ell, 0}\right|^{2}\right\| \mathbf{e}_{\ell}\left\|_{h}^{2}+\frac{1}{\eta}\right\| \varphi_{Q, h}^{\ell} \|_{h}^{2}
$$


Using this inequality into 159 one gets

$$
\begin{aligned}
-\frac{1}{V_{S}^{2} \ell} \operatorname{Im}\left(\overline{\varphi_{P}^{\ell, 0}} \varphi_{S}^{\ell, 0}\right) \geq & \left|\varphi_{P}^{\ell, 0}\right|^{2}\left(\frac{1}{V_{S}^{2}|\ell|}-\eta\left(\frac{1}{V_{P}^{2}}+\frac{1}{V_{S}^{2}}\right)\left\|\mathbf{e}_{\ell}\right\|_{h}^{2}\right) \\
& -\frac{1}{\eta}\left(\frac{1}{V_{P}^{2}}\left\|\varphi_{P, h}^{\ell}\right\|_{h}^{2}+\frac{1}{V_{S}^{2}}\left\|\varphi_{S, h}^{\ell}\right\|_{h}^{2}\right) .
\end{aligned}
$$

In consequence, from (161) and (158) we finally get

$$
\begin{aligned}
m_{\ell}\left(\boldsymbol{\varphi}_{h}^{\ell}, \boldsymbol{\varphi}_{h}^{\ell}\right) \geq & \left|\varphi_{P}^{\ell, 0}\right|^{2}\left(\frac{1}{V_{S}^{2}|\ell|}-\eta\left(\frac{1}{V_{P}^{2}}+\frac{1}{V_{S}^{2}}\right)\left\|\mathbf{e}_{\ell}\right\|_{h}^{2}\right) \\
& +\left(1-\frac{1}{\eta}\right)\left(\frac{1}{V_{P}^{2}}\left\|\varphi_{P, h}^{\ell}\right\|_{h}^{2}+\frac{1}{V_{S}^{2}}\left\|\varphi_{S, h}^{\ell}\right\|_{h}^{2}\right) .
\end{aligned}
$$

To be able to conclude, i.e., to obtain (157), it suffices to show that we can find $\eta$ such that

$$
\eta>1 \quad \text { and } \quad 1 \geq \eta\left(1+V_{S}^{2} / V_{P}^{2}\right)|\ell|\left\|\mathbf{e}_{\ell}\right\|_{h}^{2},
$$

which amounts to check that $\left(1+V_{S}^{2} / V_{P}^{2}\right)|\ell|\left\|\mathbf{e}_{\ell}\right\|_{h}^{2}<1$. We compute

$$
|\ell|\left\|\mathbf{e}_{\ell}\right\|_{h}^{2}=\frac{|\ell| h}{2}+|\ell| h \sum_{j \geq 1}^{+\infty} e^{-2|\ell| h j}=\frac{|\ell| h}{2}+|\ell| h\left(e^{2|\ell| h}-1\right)^{-1}=: g(|\ell| h),
$$

which, as a function of $|\ell| h$, can be proven to be positive and monotonically increasing in $\mathbb{R}^{+}$. Now, assuming that $L h \leq 1$ (such condition is more restrictive than (97) but still reasonable as mentioned Remark 3.10) we have

$$
\begin{aligned}
\left(1+V_{S}^{2} / V_{P}^{2}\right)|\ell|\left\|\mathbf{e}_{\ell}\right\|_{h}^{2} & \leq\left(1+V_{S}^{2} / V_{P}^{2}\right) g(1) \\
& =\left(1+V_{S}^{2} / V_{P}^{2}\right) \underbrace{\left(\frac{1}{2}+\left(e^{2}-1\right)^{-1}\right)}_{\approx 0.6565}<1
\end{aligned}
$$

where we have used that $1+V_{S}^{2} / V_{P}^{2}<3 / 2$. In consequence, we can choose $\eta$ such that 163) and therefore (157) holds for $0<\ell \leq L$.

We plot Figure 7 some snapshots of numerical results obtained with the same parameters as in Section 3.2.4 but the stable semi-discrete problem 155 is solved for $\ell \neq 0$ instead of (94). No instability has been observed in time as predicted by our analysis.

The case of $\mathbb{P}_{1}$ finite element discretizations. In more general configurations, the space $\boldsymbol{V}_{h}$ is built with $\mathbb{P}_{1}$ finite elements on triangular meshes of stepsize $h$. Notice that different meshes could be used for each potential. The space $M_{h}$ is built using $\mathbb{P}_{1}$ continuous finite elements with discontinuities at the corners and with mean value zero. Its construction is based on a mesh of the boundary obtained by restricting to $\partial \Omega$ one of the meshes used for the potentials 

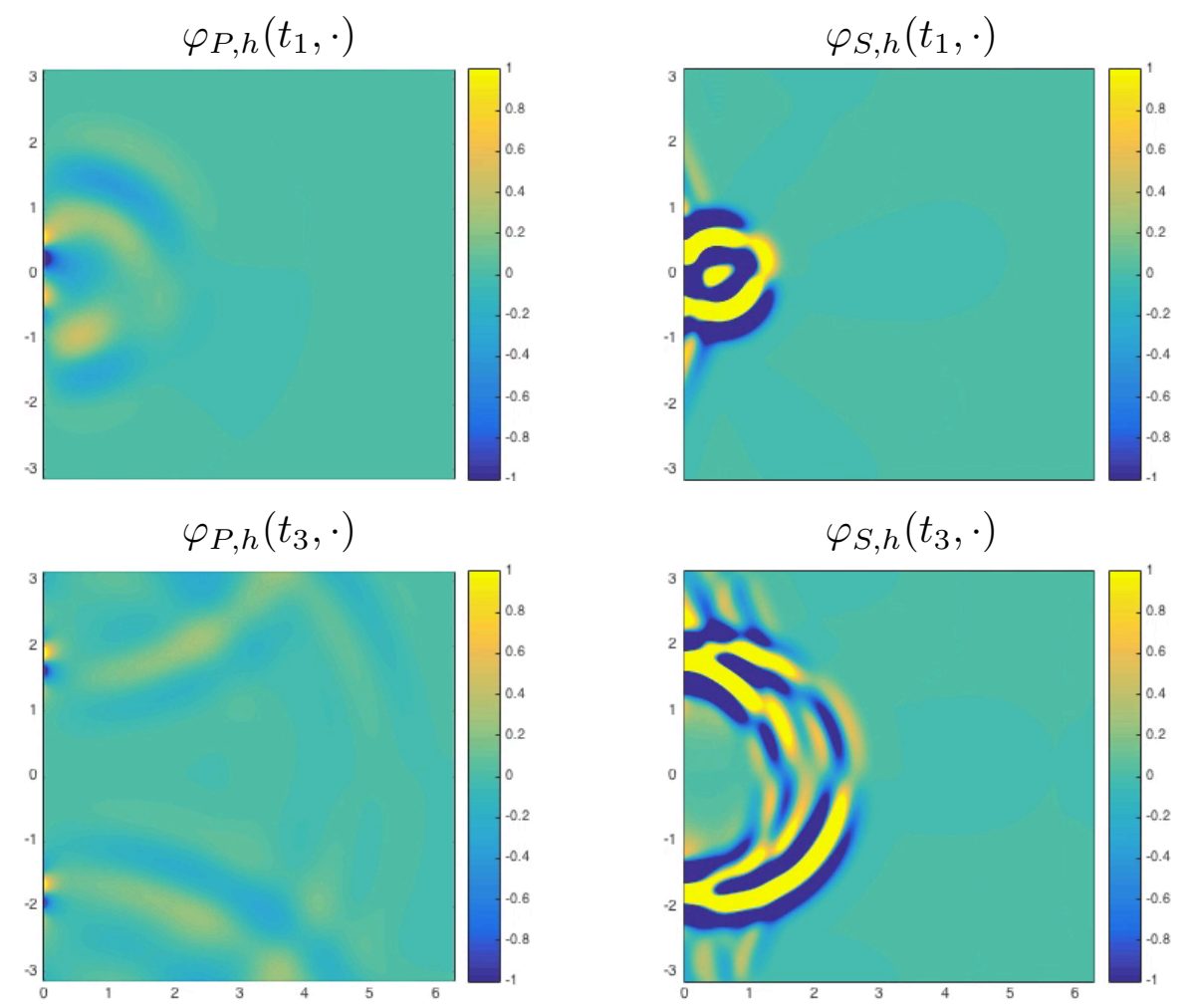

Figure 7: Snapshots at two different times $t_{1}<t_{3}$ of the solution of (146) using a spectral approximation in $x_{2}$ and $\mathbb{P}_{1}$ finite elements in $x_{1}$.

(better results are obtained when the finest one is considered). This space is generated as a Haar-like basis in which each function has a support along three adjacent segments of the boundary mesh. The discrete lifting operator $\mathcal{E}_{h}$ used in the definition of the bilinear form $b_{h}(\cdot, \cdot)$ is based in the numerical resolution of $(142)$. This is done once again using $\mathbb{P}_{1}$ finite elements. The matrices $\mathbb{M}_{R, h}$ and $\mathbb{B}_{h}$ are full rectangular matrices, $\mathbb{M}_{R, h}$ has only 3 columns, whereas the number of columns of $\mathbb{B}_{h}$ corresponds the dimension of the space $M_{h}$ and is equal to $N_{\Gamma}-1$ where $N_{\Gamma}$ is the number of nodes along the boundary. We consider that the domain of computation is $\Omega=[-5,5] \times[-5,5]$. The physical parameters are $\lambda=20, \mu=15, \rho=1$ and

$$
\boldsymbol{f}(\boldsymbol{x}, t)=\left(\begin{array}{l}
1 \\
1
\end{array}\right)\left(e^{-\left|\boldsymbol{x}-\boldsymbol{x}_{0}\right|^{2} / s_{x}}-e^{-\left|\boldsymbol{x}+\boldsymbol{x}_{0}\right|^{2} / s_{x}}\right) \partial_{t}\left(e^{-\left(t-t_{0}\right)^{2} / s_{t}}\right)
$$

with $\boldsymbol{x}_{0}=(1.5,1.5)^{t}$ and $s_{x}=0.1, t_{0}=0.8, s_{t}=0.04$. Note that $\boldsymbol{f}(\boldsymbol{x}, t)$ is constructed such that it is orthogonal to rigid motion and satisfies 60 . The spaces $V_{P, h}$ and $V_{S, h}$ are constructed using standard $\mathbb{P}_{1}$ finite elements on a 
quasi-regular mesh of approximately 16000 triangles, the time step is $\Delta t=0.01$ and the time scheme used is the explicit scheme (148). We plot Figure 8 and 9 snapshots of the obtained solution. For comparison we also plot a snapshot of the velocity field $\widetilde{\boldsymbol{v}}_{h}$ obtained by the standard $\mathbb{P}_{1}$ finite element discretization of the elastodynamics equations (2). The results obtained are stable in time, even for long time of simulations, moreover the reconstructed velocity field defined by $\boldsymbol{v}_{h}=\nabla \varphi_{P, h}+\operatorname{curl} \varphi_{S, h}-\boldsymbol{g}$ show good agreements with the direct computations of the velocity field.
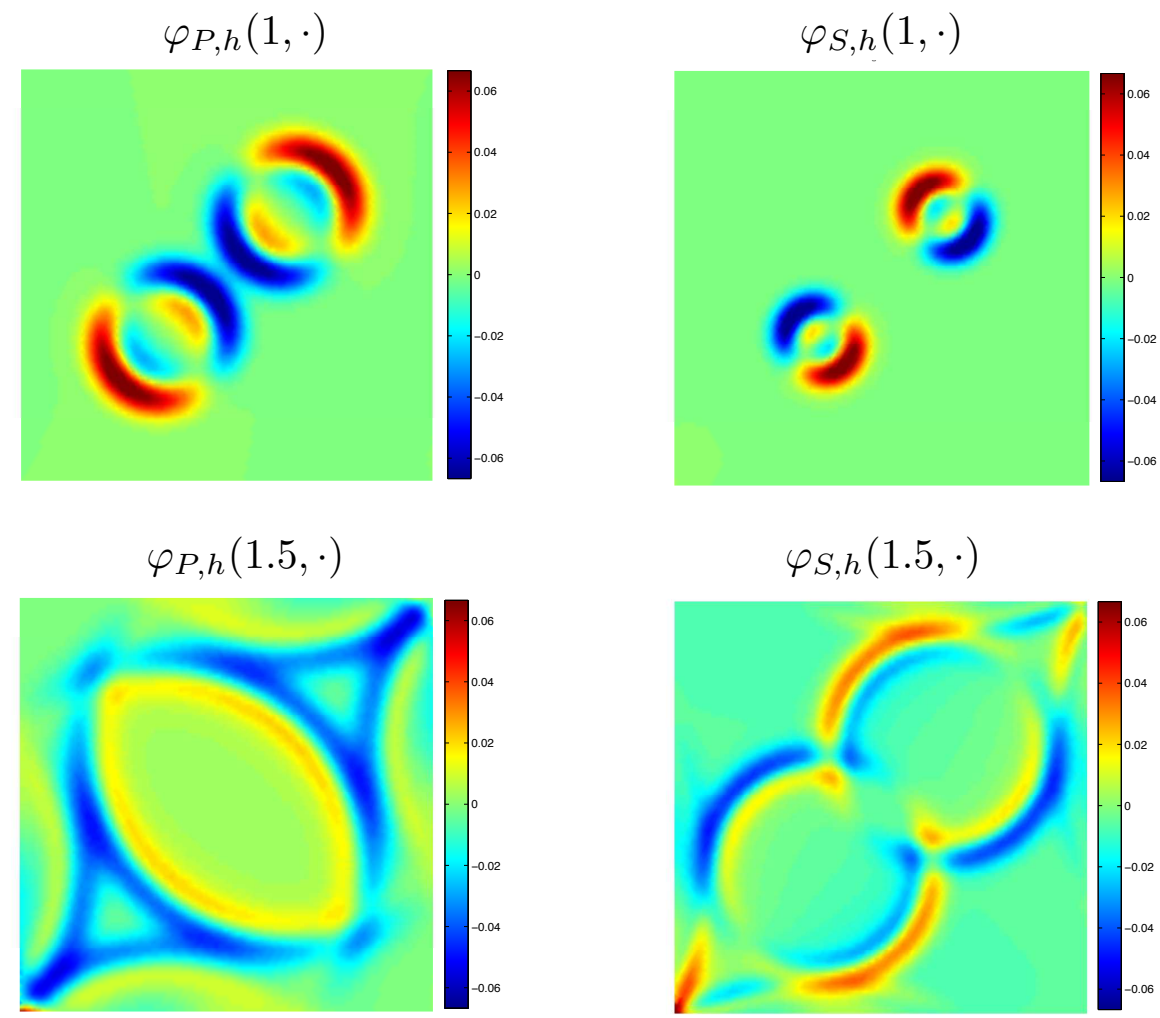

Figure 8: Snapshot of $\left(\varphi_{P, h}, \varphi_{S, h}\right)$, solution of problem (148) for different time of simulation.

\section{Conclusions and perspectives}

We have presented a method for the computation of solutions of an isotropic elastodynamics problem by solving scalar decoupled wave equations acting on the potentials of a Helmholtz decomposition of the displacement field. We detailed how these equations are coupled at the boundary and how this coupling 

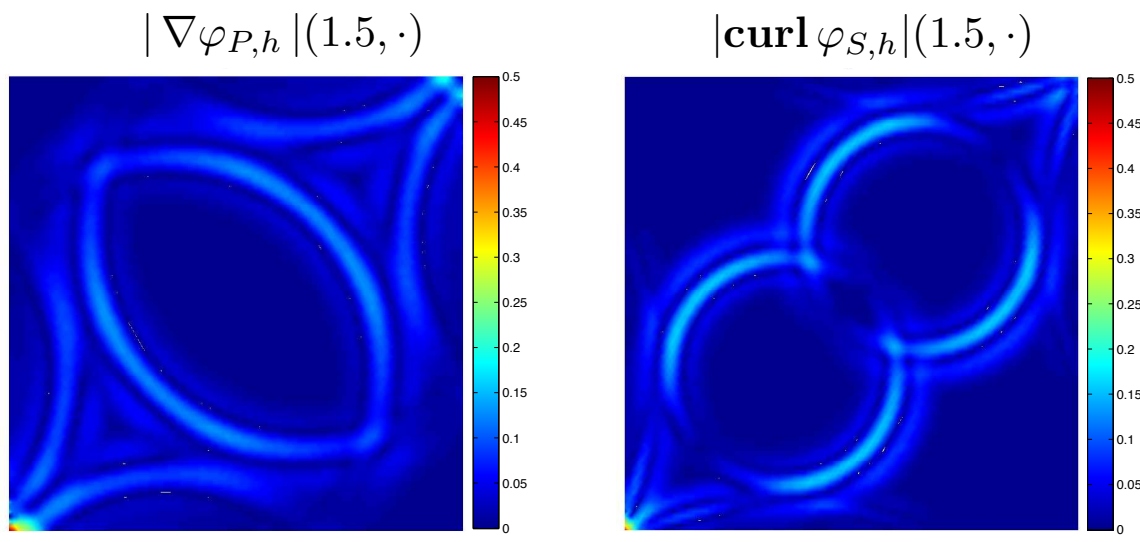

$\left|\boldsymbol{v}_{h}\right|(1.5, \cdot)$

$\left|\widetilde{\boldsymbol{v}}_{h}\right|(1.5, \cdot)$
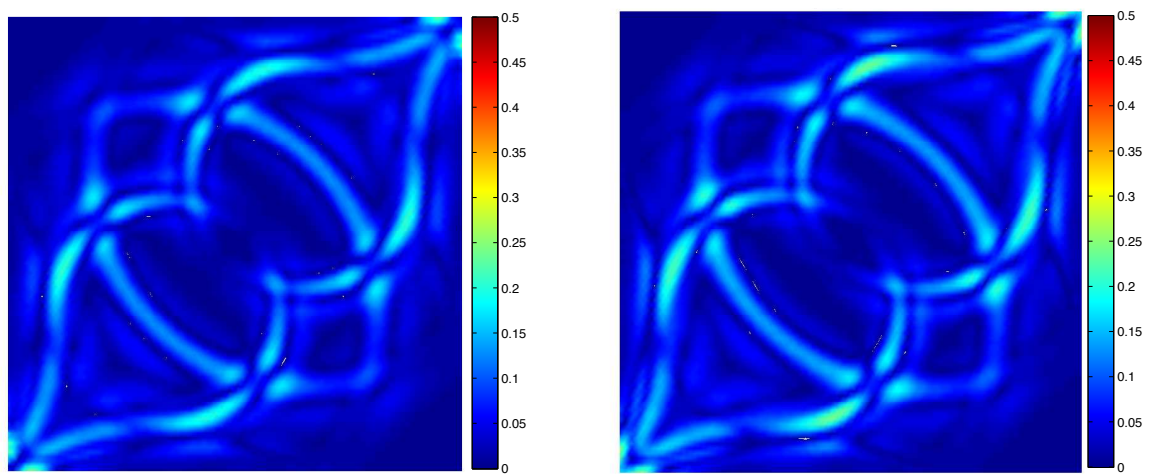

Figure 9: Snapshot of the reconstructed velocity field from the solution of problem (148) as well as the solution $\widetilde{\boldsymbol{v}}_{h}$ computed by solving (2).

takes into account the nature of the boundary conditions satisfied by the displacement field. Although the case of rigid boundary conditions presented no specific difficulty, the challenge appeared to deal with the free surface boundary conditions since severe stability issues was revealed. We solve these issues by introducing an adequate functional framework in which one has to look for the solutions to avoid these instabilities. A mixed formulation was constructed to ensure, using a Lagrange multiplier, that the sought solutions indeed belongs to the adequate functional space. Numerical results confirm the stability of the proposed approach. Among the perspectives of this work we can mention, first, the construction and analysis of an efficient numerical method, including the analysis of the discrete lifting operator introduced in the last section. Then, the analysis of the transmission problem between two isotropic media could be addressed, however we do not expect theoretical difficulties since one can see transmission conditions as both heterogeneous Dirichlet and Neumann bound- 
ary conditions. Finally, the 3D case should be addressed, the difficulty being that the potential corresponding to the shear waves in the Helmholtz decomposition is no longer scalar and is associated with a gauge condition that should be taken into account.

Acknowledgments The research of the first and fourth authors was partially funded by FEDER and the Spanish Ministry of Science and Innovation through grants MTM2013-43745-R and MTM2017-86459-R and by Xunta de Galicia through grant ED431C 2017/60.

\section{References}

[1] J. Virieux. P-SV wave propagation in heterogeneous media: Velocity- stress finite-diference method. Geophysics, 51(4):889-901, 1986.

[2] Girault V. and Raviart P.-A. Finite element methods for Navier-Stokes equations: theory and algorithms, volume 5. Springer Science \& Business Media, 2012.

[3] Glowinski R. and Pironneau O. Numerical methods for the first biharmonic equation and the two-dimensional Stokes problem. SIAM Rev., 21(2):167$212,1979$.

[4] Babuska I., Osborn J., and Pitkäranta J. Analysis of mixed methods using mesh dependent norms. Mathematics of Computation, 35(152):1039-1062, 1980.

[5] Komatitsch D. and Martin R. An unsplit convolutional perfectly matched layer improved at grazing incidence for the seismic wave equation. Geophysics, 72(5):SM155-SM167, 2007.

[6] Burel A., Imperiale S., and Joly P. Solving the homogeneous isotropic linear elastodynamics equations using potentials and finite elements. The case of the rigid boundary condition. Numerical Analysis and Applications, 5(2):136-143, 2012.

[7] Burel A. Contributions à la simulation numérique en élastodynamique: découplage des ondes $P$ et $S$, modèles asymptotiques pour la traversée de couches minces. PhD thesis, Université Paris Sud-Paris XI, 2014.

[8] Gurtin M. E. An introduction to continuum mechanics, volume 158. Academic press, 1982.

[9] Ciarlet P. G. Elasticité tridimensionnelle, volume 1. Masson, 1986.

[10] Alonso Rodríguez A. and Valli A. Eddy Current Approximation of Maxwell Equations: Theory, Algorithms and Applications, volume 4. Springer Science \& Business Media, 2010. 
[11] Lions J.-L. and E. Magenes. Non-Homogeneous Boundary Value Problems and Applications, volume 1. Springer Berlin Heidelberg, 1972.

[12] Monk P. Finite Element Methods for Maxwell's Equations. Oxford University Press, 2003.

[13] Cherif A., Bernardi C., Dauge M., and Girault V. Vector potentials in threedimensional non-smooth domains. Mathematical Methods in the Applied Sciences, 21(9):823-864, 1998.

[14] Ciarlet P. G. The finite element method for elliptic problems. SIAM, 2002.

[15] Cohen G. Higher-Order Numerical Methods for Transient Wave Equations. Springer, 2002.

[16] Komatitsch D. and Tromp J. Introduction to the spectral element method for three-dimensional seismic wave propagation. Geophysical Journal International, 139(3):806-822, 1999.

[17] Cohen G., Joly P., Roberts J. E., and Tordjman N. Higher order triangular finite elements with mass lumping for the wave equation. SIAM Journal on Numerical Analysis, 38(6):2047-2078, 2001.

[18] Ciarlet P. G. On Korn's inequality. Chinese Annals of Mathematics-Series $B, 31(5): 607-618,2010$.

[19] Pavel Bochev and Richard B Lehoucq. On the finite element solution of the pure Neumann problem. SIAM review, 47(1):50-66, 2005.

[20] Alfredo Bermúdez de Castro, Dolores Gómez, and Pilar Salgado. Mathematical models and numerical simulation in electromagnetism, volume 74 . Springer, 2014.

[21] Wei Jiang, Na Liu, Yifa Tang, and Qing Huo Liu. Mixed finite element method for 2d vector Maxwell's eigenvalue problem in anisotropic media. Progress In Electromagnetics Research, 148:159-170, 2014.

[22] Franco Brezzi and Michel Fortin. Mixed and Hybrid Finite Element Methods. Springer-Verlag New York, Inc., New York, NY, USA, 1991. 